\title{
Total Synthesis of 10-Deoxymethynolide and Narbonolide
}

\section{SUPPORTING INFORMATION}

\author{
Richeng Xuan, ${ }^{\dagger}$ Hong-Se Oh,$^{\dagger}$ Younghoon Lee, ${ }^{\dagger}$ and Han-Young Kang, ${ }^{*},{ }^{\dagger}$ \\ Department of Chemistry, Chungbuk National University, Cheongju, Chungbuk 361-763, Korea and \\ Department of Chemistry and Center for Molecular Design and Synthesis, Korea Advanced Institute of
}

Science and Technology, Daejeon 305-701, Korea

hykang@chungbuk.ac.kr

${ }^{\dagger}$ Chungbuk National University
${ }^{\ddagger}$ Korea Advanced Institute of Science and Technology

Contents

Experimental

General 


\section{Experimental}

General

All ${ }^{1} \mathrm{H}$ NMR and ${ }^{13} \mathrm{C}$ NMR spectra were recorded at ambient temperature, chemical shifts $\mathrm{d}$ are give in ppm, coupling constant $\mathrm{J}$ are in $\mathrm{Hz}$. The chemical shifts are reported in ppm on scale downfield from TMS, and signal patterns are indicated as follows: s, singlet; d, doublet; $\mathrm{t}$, triplet; $\mathrm{m}$, multiplet; br, broad peak. IR specta were recorded as thin films. Optical rotations were measured using a $1 \mathrm{~mL}$ cell with a $1 \mathrm{dm}$ path length, the concentration $c$ is given in $\mathrm{g} / 100 \mathrm{~mL}$. Mass spectra (and HRMS) were obtained on VG AUTOSPEC Ultma GC/MS system using direct insertion probe(DIP) and electron impact (EI) $(70 \mathrm{eV})$ method or on 4.7 Telsa IonSpec ESI-FTMS or a Micromass LCT ESI-TOF mass spectrometer. All reagent and solvents were reagent grade and used without further purification unless specified otherwise. Technical grade ethyl acetate, hexane, and pentane used for column chromatography were distilled prior to use. Tetrahydrofuran (THF) and diethyl ether, when used as solvents for reactions, were freshly distilled from sodium-benzophenone ketyl. Dimethylformide (DMF) was stored over 4- $\AA$ molecular sieves, and triethylamine was distilled before use. Flash chromatography was carried out using silica gel $60(0.040-0.063 \mathrm{~mm})$ packed in glass columns. All reactions were performed under an atmosphere of nitrogen in flame-dried 
or oven-dried glassware with magnetic stirring.

(2S,4R)-5-(4-Methoxybenzyloxy)-2,4-dimethylpentan-1-ol (11c)

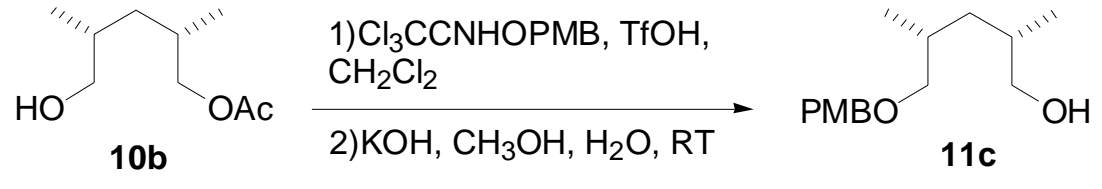

To a stirred solution of alcohol $\mathbf{1 0 b}(1.55 \mathrm{~g}, 8.90 \mathrm{mmol})$ and 4-methoxybenzyl 2,2,2trichloroacetimidate $(5.78 \mathrm{~g}, 20.5 \mathrm{mmol})$ in $\mathrm{CH}_{2} \mathrm{Cl}_{2}(25 \mathrm{~mL})$ was added a solution of trifluoromethanesulfonic acid (10 drops) in ether $(10 \mathrm{~mL})$ dropwise via a cannula at $0{ }^{\circ} \mathrm{C}$. After $30 \mathrm{~min}$ aqueous saturated $\mathrm{NaHCO}_{3}(20 \mathrm{~mL})$ was added and the mixture was extracted with $\mathrm{CH}_{2} \mathrm{Cl}_{2}(3 \times 20 \mathrm{~mL})$. The organic phase was washed with $\mathrm{H}_{2} \mathrm{O}(20 \mathrm{~mL})$, dried $\left(\mathrm{MgSO}_{4}\right)$ and concentrated. Purification by flash chromatography (hexane:EtOAc $=10: 1)$ provided PMB-protected acetate $(2.62 \mathrm{~g}, 100 \%)$ as a colorless oil.

To a solution of the PMB-protected acetate $(2.51 \mathrm{~g}, 8.53 \mathrm{mmol})$ in $\mathrm{CH}_{3} \mathrm{OH}(20 \mathrm{~mL})$ at room temperature was added an aqueous solution of $\mathrm{KOH}(1.10 \mathrm{~g}, 19.6 \mathrm{mmol})$ in $\mathrm{H}_{2} \mathrm{O}$ $(10 \mathrm{~mL})$ dropwise. The reaction mixture was stirred for $30 \mathrm{~min}$. The reaction was terminated by the addition of aqueous saturated $\mathrm{NH}_{4} \mathrm{Cl}(30 \mathrm{~mL})$. The mixture was extracted with $\mathrm{CH}_{2} \mathrm{Cl}_{2}(3 \times 20 \mathrm{~mL})$. The organic layer was dried $\left(\mathrm{MgSO}_{4}\right)$, filtered, and concentrated. Purification by flash chromatography (hexane:EtOAc $=2: 1$ ) afforded the 
desired alcohol $11(1.54 \mathrm{~g}, 72 \%)$ as a colorless oil: $[\alpha]_{\mathrm{D}}{ }^{20.5}-3.85^{\circ}\left(\mathrm{c} 1.08, \mathrm{CHCl}_{3}\right) ;{ }^{1} \mathrm{H}$ NMR (300 MHz, $\left.\mathrm{CDCl}_{3}\right) \delta 0.93(\mathrm{~d}, J=6.7 \mathrm{~Hz}, 3 \mathrm{H}), 0.94(\mathrm{~d}, J=6.7 \mathrm{~Hz}, 3 \mathrm{H}), 0.90-1.00$ (m, 1H), 1.41-1.50 (m, 1H), 1.65-1.75 (m, 1H), 1.79-1.87 (m, 2H), $3.22(\mathrm{dd}, J=9.0,6.5$ $\mathrm{Hz}, 1 \mathrm{H}), 3.32(\mathrm{dd}, J=9.0,5.8 \mathrm{~Hz}, 1 \mathrm{H}), 3.41(\mathrm{dd}, J=10.6,6.2 \mathrm{~Hz}, 1 \mathrm{H}), 3.48(\mathrm{dd}, J=$ 10.6, 5.2 Hz, 1H), 3.81 (s, 3H), $4.42(\mathrm{~s}, 2 \mathrm{H}), 6.80-6.95(\mathrm{~m}, 2 \mathrm{H}), 7.20-7.30(\mathrm{~m}, 2 \mathrm{H}) ;{ }^{13} \mathrm{C}$ $\operatorname{NMR}\left(75 \mathrm{MHz}, \mathrm{CDCl}_{3}\right) \delta 158.9,130.5,129.0,113.6,75.5,72.5,67.5,55.1,38.5,33.0$, $30.8,18.0,17.5$.

(R)-4-Benzyl-3-[(2R,3S,4S,6R)-3-hydroxy-7-(4-methoxybenzyloxy)-2,4,6-trimethylheptanoyl]-oxazolidin-2-one (13)<smiles>CCCCOC[C@H](C)C[C@H](C)CO</smiles>

$11 \mathrm{c}$
1) $\mathrm{SO}_{3} \cdot \mathrm{Py}, \mathrm{DMSO}, \mathrm{Et}_{3} \mathrm{~N}$

2) 12, $\mathrm{Bu}_{2} \mathrm{BOTf}, \mathrm{Et}_{3} \mathrm{~N}, \mathrm{CH}_{2} \mathrm{Cl}_{2}$ $-78{ }^{\circ} \mathrm{C} \sim$ R.T; $\mathrm{H}_{2} \mathrm{O}_{2}$

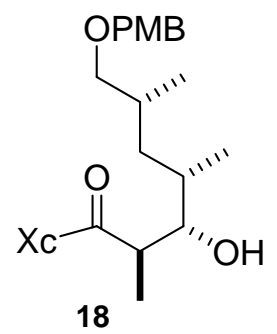

To a stirred solution of alcohol 11c $(122 \mathrm{mg}, 0.483 \mathrm{mmol})$ and triethylamine $(673 \mu \mathrm{L}$, $4.83 \mathrm{mmol})$ in $\mathrm{CH}_{2} \mathrm{Cl}_{2}(1.5 \mathrm{~mL})$, and, was added a solution of pyridine- $\mathrm{SO}_{3}$ complex (253 $\mathrm{mg}, 1.59 \mathrm{mmol})$ in DMSO $(1.5 \mathrm{~mL})$ at room temperature. The mixture was stirred for $1 \mathrm{~h}$ at room temperature. The reaction was terminated by addition of aqueous 
saturated $\mathrm{NaCl}(2 \mathrm{~mL})$. The mixture was extracted with $\mathrm{CH}_{2} \mathrm{Cl}_{2}(3 \times 10 \mathrm{~mL})$ and the organic layer was dried $\left(\mathrm{MgSO}_{4}\right)$ and concentrated. Purification by flash chromatography (hexane:EtOAc $=5: 1)$ afforded the desired aldehyde $(105 \mathrm{mg}, 87 \%)$ as a colorless oil: $[\alpha]_{\mathrm{D}}{ }^{25.2}+7.21^{\circ}\left(c 1.04, \mathrm{CHCl}_{3}\right) ;{ }^{1} \mathrm{H}$ NMR $\left(300 \mathrm{MHz}, \mathrm{CDCl}_{3}\right) \delta 0.94(\mathrm{~d}, J$ $=6.7 \mathrm{~Hz}, 3 \mathrm{H}), 1.09(\mathrm{~d}, J=6.9 \mathrm{~Hz}, 3 \mathrm{H}), 1.05-1.21(\mathrm{~m}, 1 \mathrm{H}), 1.75-1.95(\mathrm{~m}, 2 \mathrm{H}), 2.40-$ $2.60(\mathrm{~m}, 1 \mathrm{H}), 3.26(\mathrm{~d}, J=5.7 \mathrm{~Hz}, \quad 2 \mathrm{H}), \quad 3.81(\mathrm{~s}, 3 \mathrm{H}), 4.41(\mathrm{~s}, 2 \mathrm{H}), 6.85-6.92(\mathrm{~m}, 2 \mathrm{H})$, 7.20-7.30 (m, 2H), $9.56(\mathrm{~d}, J=2.5 \mathrm{~Hz}, 1 \mathrm{H}) ;{ }^{13} \mathrm{C} \mathrm{NMR}\left(75 \mathrm{MHz}, \mathrm{CDCl}_{3}\right) \delta$ 205.1, 159.0, $130.5,129.0,113.6,74.9,72.5,55.1,44.0,35.0,31.1,17.5,14.2$.

To a solution of (4R)-3-propionyl-4-benzyl-2-oxazolidinone (12) (1.45 g, $6.23 \mathrm{mmol})$ in $\mathrm{CH}_{2} \mathrm{Cl}_{2}(15 \mathrm{~mL})$ was added dibutylboron triflate $\left(6.48 \mathrm{~mL}\right.$ of a $1.0 \mathrm{M}$ solution in $\mathrm{CH}_{2} \mathrm{Cl}_{2}$, $6.48 \mathrm{mmol})$ and triethylamine $(1.00 \mathrm{~mL}, 7.26 \mathrm{mmol})$ dropwise at $0{ }^{\circ} \mathrm{C}$. The solution was cooled down to $-78{ }^{\circ} \mathrm{C}$. To this solution was added a solution of the aldehyde prepared in the procedure describe above $(1.30 \mathrm{~g}, 5.19 \mathrm{mmol})$ in $\mathrm{CH}_{2} \mathrm{Cl}_{2}(5 \mathrm{~mL})$ at $-78{ }^{\circ} \mathrm{C}$. The resulting solution was stirred for $20 \mathrm{~min}$ at $-78{ }^{\circ} \mathrm{C}$. The solution was then warmed to $0{ }^{\circ} \mathrm{C}$ and stirred for $1 \mathrm{~h}$ additionally. The reaction was terminated by adding a $\mathrm{pH} 7$ aqueous phosphate buffer solution (0.2 M-sodium hydrogen phosphate:0.1 M-citric acid $=82: 18,10 \mathrm{~mL})$ and methanol $(30 \mathrm{~mL})$. To this cloudy solution was added a solution of 
methanol and $30 \%$ hydrogen peroxide $(2: 1,30 \mathrm{~mL})$ and the resulting solution was stirred for $1 \mathrm{~h}$ at $0{ }^{\circ} \mathrm{C}$. After the solution was concentrated, it was extracted with diethyl ether $(3 \times 20 \mathrm{~mL})$. The organic layer was washed with saturated aqueous sodium bicarbonate $(25 \mathrm{~mL})$ and saturated aqueous $\mathrm{NaCl}(25 \mathrm{~mL})$, dried $\left(\mathrm{MgSO}_{4}\right)$, and concentrated. Purification of the residue by flash chromatography (hexane:EtOAc $=4: 1$ ) provided the desired aldol product $18(1.68 \mathrm{~g}, 67 \%)$ as a white solid: $\mathrm{mp} 68.5-69.2{ }^{\circ} \mathrm{C}$; $[\alpha]_{\mathrm{D}}^{27.6}-39.2^{\circ}\left(c 1.20, \mathrm{CHCl}_{3}\right)$; IR (film) $v_{\max } 3529,2958,1778,1689,1612,1511,1457$, 1380, 1241, 1059, 979, 825, 748, $702 \mathrm{~cm}^{-1} ;{ }^{1} \mathrm{H}$ NMR $\left(300 \mathrm{MHz}, \mathrm{CDCl}_{3}\right) \delta 0.90(\mathrm{~d}, J=$ $6.6 \mathrm{~Hz}, 3 \mathrm{H}), 0.90-1.05(\mathrm{~m}, 1 \mathrm{H}), 0.97(\mathrm{~d}, J=6.6 \mathrm{~Hz}, 3 \mathrm{H}), 1.21(\mathrm{~d}, J=6.9 \mathrm{~Hz}, 3 \mathrm{H}), 1.60-$ $1.72(\mathrm{~m}, 1 \mathrm{H}), 1.77$ (ddd, $J=13.1,9.0,3.3 \mathrm{~Hz}, 1 \mathrm{H}), 1.86-2.00(\mathrm{~m}, 1 \mathrm{H}), 2.79(\mathrm{dd}, J=$ 13.3, $9.5 \mathrm{~Hz}, 1 \mathrm{H}), 2.87(\mathrm{~s}, 1 \mathrm{H}), 3.21(\mathrm{dd}, \quad J=9.1,6.8 \mathrm{~Hz}, 1 \mathrm{H}), 3.26(\mathrm{dd}, J=12.8,3.1$ Hz, 1H), $3.36(\mathrm{dd}, J=9.1,5.2 \mathrm{~Hz}, 1 \mathrm{H}), 3.58(\mathrm{~d}, J=7.1 \mathrm{~Hz}, 1 \mathrm{H}), 3.79(\mathrm{~s}, 3 \mathrm{H}), 3.95$ (dddd, $J=6.9,6.9,6.9,2.6 \mathrm{~Hz}, 1 \mathrm{H}), 4.15(\mathrm{~d}, J=5.0 \mathrm{~Hz}, 2 \mathrm{H}), 4.42(\mathrm{dd}, J=15.7,11.7$ $\mathrm{Hz}, 2 \mathrm{H}), 4.64(\mathrm{~m}, 1 \mathrm{H}), 6.87(\mathrm{~d}, J=11.3 \mathrm{~Hz}, 2 \mathrm{H}), 7.19-7.37(\mathrm{~m}, 7 \mathrm{H}) ;{ }^{13} \mathrm{C}$ NMR $(75$ $\left.\mathrm{MHz}, \mathrm{CDCl}_{3}\right) \delta 177.5,159.0,152.8,135.0,130.7,129.3,129.0,128.9,127.3,113.6$, 76.0, 75.3, 72.6, 66.0, 55.2, 39.7, 38.0, 37.7, 33.9, 31.2, 19.2, 16.3, 9.8; HRMS calcd for $\mathrm{C}_{28} \mathrm{H}_{37} \mathrm{NO}_{6} 483.2621$, found 483.2621 . 
(2R,3S,4S,6R)-3-(tert-Butyldimethylsilanyloxy)-7-(4-methoxybenzyloxy)-2,4,6-

\section{trimethylheptanoic acid (19)}

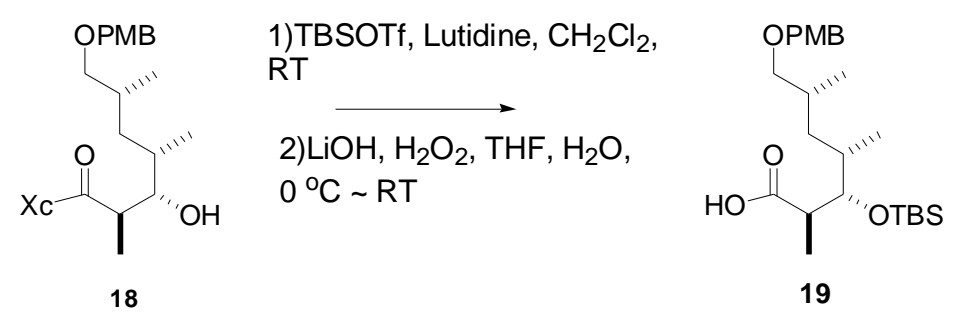

To a stirred solution of aldol product $18(514 \mathrm{mg}, 1.06 \mathrm{mmol}), 2$,6-lutidine $(222 \mu \mathrm{L}$, $1.91 \mathrm{mmol})$ in $\mathrm{CH}_{2} \mathrm{Cl}_{2}(5.0 \mathrm{~mL})$ was added TBSOTf $(360 \mu \mathrm{L}, 1.59 \mathrm{mmol})$ at $0{ }^{\circ} \mathrm{C}$. After the solution was stirred for $4 \mathrm{~h}$, the reaction was terminated by adding saturated aqueous $\mathrm{NaCl}(5 \mathrm{~mL})$ and aqueous saturated $\mathrm{NH}_{4} \mathrm{Cl}(5 \mathrm{~mL})$. The organic layer was separated and the aqueous layer was extracted with $\mathrm{CH}_{2} \mathrm{Cl}_{2}(3 \times 5 \mathrm{~mL})$. The organic solutions were combined, dried $\left(\mathrm{MgSO}_{4}\right)$ and concentrated. Purification of the residue by flash chromatography (hexane:EtOAc $=5: 1$ ) afforded the TBS-protected product $(565 \mathrm{mg}$, $89 \%$ ) as a colorless oil: $[\alpha]_{\mathrm{D}}^{25.9}-37.1^{\circ}\left(c 1.36, \mathrm{CHCl}_{3}\right)$; IR (film) $v_{\max } 2954,2850,1781$, $1612,1511,1461,1380,1245,1095,968,837,771 \mathrm{~cm}^{-1} ;{ }^{1} \mathrm{H} \mathrm{NMR}\left(300 \mathrm{MHz}, \mathrm{CDCl}_{3}\right) \delta$ $0.06(\mathrm{~s}, 3 \mathrm{H}), 0.08(\mathrm{~s}, 3 \mathrm{H}), 0.80-1.00(\mathrm{~m}, 16 \mathrm{H}), 1.22(\mathrm{~d}, J=6.2 \mathrm{~Hz}, 3 \mathrm{H}), 1.39$ (ddd, $J=$ 13.6, 7.9, $5.2 \mathrm{~Hz}, 1 \mathrm{H}), 1.70-1.90(\mathrm{~m}, 2 \mathrm{H}), 1.71(\mathrm{dd}, J=13.3,9.6 \mathrm{~Hz}, 1 \mathrm{H}), 3.16(\mathrm{dd}, J=$ 9.1, $6.7 \mathrm{~Hz}, 1 \mathrm{H}), 3.25(\mathrm{dd}, J=9.1,5.7 \mathrm{~Hz}, 1 \mathrm{H}), 3.10-3.30(\mathrm{~m}, 1 \mathrm{H}), 3.76(\mathrm{~s}, 3 \mathrm{H}), 3.89$ $(\mathrm{dd}, J=8.7,8.7 \mathrm{~Hz}, 1 \mathrm{H}), 3.95-4.10(\mathrm{~m}, 3 \mathrm{H}), 4.37(\mathrm{~s}, 2 \mathrm{H}), 4.46(\mathrm{~m}, 1 \mathrm{H}), 6.81(\mathrm{~m}, 2 \mathrm{H})$, 
7.10-7.40 (m, 7H); ${ }^{13} \mathrm{C}$ NMR $\left(75 \mathrm{MHz}, \mathrm{CDCl}_{3}\right) \delta 176.1,158.9,152.8,135.3,130.7$, $129.4,129.0,128.8,127.2,113.6,75.9,75.8,72.5,65.7,55.4,55.1,40.1,37.5,36.8$, 36.4, 30.5, 26.0, 18.6, 18.3, 16.4, 14.8, -3.8, -4.1; HRMS calcd for $\mathrm{C}_{34} \mathrm{H}_{51} \mathrm{NO}_{6} \mathrm{Si}$ 597.3486, found 597.3464.

To a stirred solution of the TBS-protected product $(565 \mathrm{mg}, 0.945 \mathrm{mmol}$ ) in tetrahydrofuran-water $(4: 1,13 \mathrm{~mL})$ was added $30 \%$ aqueous hydrogen peroxide $(500$ $\mu \mathrm{L}, 4.40 \mathrm{mmol})$ and $0.8 \mathrm{M}$ aqueous lithium hydroxide $(2.0 \mathrm{~mL}, 1.6 \mathrm{mmol})$ at $0{ }^{\circ} \mathrm{C}$. After $5 \mathrm{~h}$, the reaction was terminated by adding aqueous $\mathrm{Na}_{2} \mathrm{SO}_{3}(1.33 \mathrm{M}$ solution, $3.0 \mathrm{~mL}$, $4.0 \mathrm{mmol})$ and aqueous saturated $\mathrm{NH}_{4} \mathrm{Cl}(20 \mathrm{~mL})$. The resulting mixture was extracted with ether $(3 \times 20 \mathrm{~mL})$. The organic layer was dried $\left(\mathrm{MgSO}_{4}\right)$ and concentrated. Purification of the residue by flash chromatography (hexane:EtOAc $=3: 1$ ) afforded the desired carboxylic acid $19(361 \mathrm{mg}, 87 \%)$ as a colorless oil: $[\alpha]_{\mathrm{D}}^{26.3}-9.8^{\circ}(c$ 1.30, $\mathrm{CHCl}_{3}$ ); IR (film) $v_{\max } 2950,1704,1612,1511,1462,1250,1173,1088,833,775 \mathrm{~cm}^{-1}$; ${ }^{1} \mathrm{H}$ NMR (300 MHz, $\left.\mathrm{CDCl}_{3}\right) \delta 0.03(\mathrm{~s}, 3 \mathrm{H}), 0.06(\mathrm{~s}, 3 \mathrm{H}), 0.89(\mathrm{~s}, 9 \mathrm{H}), 0.92(\mathrm{~d}, J=6.9$ $\mathrm{Hz}, 3 \mathrm{H}), 0.96(\mathrm{~d}, J=6.6 \mathrm{~Hz}, 3 \mathrm{H}), 0.90-1.10(\mathrm{~m}, 1 \mathrm{H}), 1.15(\mathrm{~d}, J=7.0 \mathrm{~Hz}, 3 \mathrm{H}), 1.50$ (ddd, $J=4.5,8.4,13.5 \mathrm{~Hz}, 1 \mathrm{H}), 1.70-1.90(\mathrm{~m}, 2 \mathrm{H}), 2.63(\mathrm{~m}, 1 \mathrm{H}), 3.16(\mathrm{dd}, J=7.0,9.0 \mathrm{~Hz}$, $1 \mathrm{H}), 3.31(\mathrm{dd}, J=4.9,9.0 \mathrm{~Hz}, 1 \mathrm{H}), 3.80(\mathrm{~s}, 3 \mathrm{H}), 3.90(\mathrm{t}, J=4.6 \mathrm{~Hz}, 1 \mathrm{H}), 4.41(\mathrm{dd}, J=$ 
13.4, $11.8 \mathrm{~Hz}, 2 \mathrm{H}), 6.87(\mathrm{~d}, J=8.6 \mathrm{~Hz}, 2 \mathrm{H}), 7.25(\mathrm{~d}, J=8.5 \mathrm{~Hz}, 2 \mathrm{H}) ;{ }^{13} \mathrm{C}$ NMR $(75$ $\left.\mathrm{MHz}, \mathrm{CDCl}_{3}\right) \delta 181.2,159.0,130.7,129.0,113.6,76.5,75.1,72.6,55.2,42.2,36.9$, $36.1,30.9,25.9,20.4,18.9,18.2,16.2,12.1,-4.2,-4.3$. HRMS calcd for $\mathrm{C}_{24} \mathrm{H}_{42} \mathrm{O}_{5} \mathrm{Si}$ 438.2802, found 438.2808 .

$(1 R, 2 R)-1-e t h y l-2-m e t h y l-b u t-3-e n y l ~(2 R, 3 S, 4 S, 6 R)-3-(t e r t-$

butyldimethylsilanyloxy)-7-(4-methoxybenzyloxy)-2,4,6-trimethylheptanoate (20)

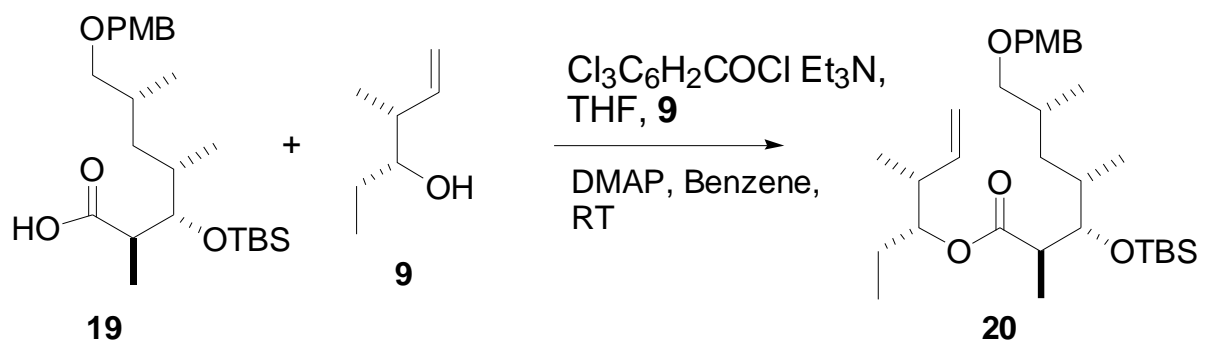

To a solution of carboxylic acid $19(200 \mathrm{mg}, 0.456 \mathrm{mmol})$ in THF (3 mL) at room temperature were added triethylamine $(90 \mu \mathrm{L}, 0.63 \mathrm{mmol})$ and 2,4,6-trichlorobenzoyl chloride $(94 \mu \mathrm{L}, 0.60 \mathrm{mmol})$. The mixture was stirred for $3 \mathrm{~h}$ at room temperature, and the solids were filtered off and washed with hexane $(5 \mathrm{~mL})$. The combined solution was concentrated under reduced pressure. The residue was dissolved in benzene $(2 \mathrm{~mL})$, and to this solution a solution of alcohol $9(83.3 \mathrm{mg}, 0.729 \mathrm{mmol})$ and DMAP $(77.9 \mathrm{mg}$, $0.638 \mathrm{mmol})$ in benzene $(2 \mathrm{~mL})$ was added. After being stirred for $13 \mathrm{~h}$, the reaction mixture was diluted with ether $(20 \mathrm{~mL})$, and washed with saturated $\mathrm{NaHCO}_{3}(10 \mathrm{~mL})$ 
and saturated $\mathrm{NaCl}(10 \mathrm{~mL})$, dried $\left(\mathrm{MgSO}_{4}\right)$, and concentrated. Purification of the residue by flash chromatography (hexane:EtOAc $=9: 1$ ) afforded the desired ester 20 (194 mg, 79\%) as a colorless oil: $[\alpha]_{\mathrm{D}}^{25.0}+11.6^{\circ}\left(c\right.$ 1.11, $\left.\mathrm{CHCl}_{3}\right)$; IR (film) $v_{\max } 2958$, $1728,1612,1512,1462,1373,1250,1173,1057,910,833,775 \mathrm{~cm}^{-1} ;{ }^{1} \mathrm{H}$ NMR $(300$ $\left.\mathrm{MHz}, \mathrm{CDCl}_{3}\right) \delta 0.04(\mathrm{~s}, 3 \mathrm{H}), 0.05(\mathrm{~s}, 3 \mathrm{H}), 0.85(\mathrm{t}, J=7.5 \mathrm{~Hz}, 3 \mathrm{H}), 0.89(\mathrm{~s}, 9 \mathrm{H}), 0.92(\mathrm{~d}$, $J=6.9 \mathrm{~Hz}, 3 \mathrm{H}), 0.96(\mathrm{~d}, J=6.6 \mathrm{~Hz}, 3 \mathrm{H}), 1.00(\mathrm{~d}, J=6.8 \mathrm{~Hz}, 3 \mathrm{H}), 0.90-1.10(\mathrm{~m}, 1 \mathrm{H})$, $1.15(\mathrm{~d}, J=7.0 \mathrm{~Hz}, 3 \mathrm{H}), 1.40(\mathrm{ddd}, J=4.5,8.1,13.0 \mathrm{~Hz}, 1 \mathrm{H}), 1.45-1.60(\mathrm{~m}, 2 \mathrm{H}), 1.62-$ $1.75(\mathrm{~m}, 1 \mathrm{H}), 1.75-1.90(\mathrm{~m}, 2 \mathrm{H}), 2.43$ (ddddd, $J=6.7,6.7,6.7,6.7,6.7 \mathrm{~Hz}, 1 \mathrm{H}), 2.58$ (dddd, $J=7.0,7.0,7.0,7.0 \mathrm{~Hz}, 1 \mathrm{H}), 3.11(\mathrm{dd}, J=9.0,7.4 \mathrm{~Hz}, 1 \mathrm{H}), 3.33(\mathrm{dd}, J=9.0,4.6$ $\mathrm{Hz}, 1 \mathrm{H}), 3.80(\mathrm{~s}, 3 \mathrm{H}), 3.88(\mathrm{dd}, J=6.4,3.3 \mathrm{~Hz}, 1 \mathrm{H}), 4.41(\mathrm{~s}, 2 \mathrm{H}), 4.72(\mathrm{ddd}, J=7.4,6.0$, $4.7 \mathrm{~Hz}, 1 \mathrm{H}), 5.04(\mathrm{~m}, 2 \mathrm{H}), 5.74$ (ddd, $J=17.3,10.4,7.4 \mathrm{~Hz}, 1 \mathrm{H}), 6.86$ (d, $J=8.6 \mathrm{~Hz}$, 2H), $7.25(\mathrm{~d}, J=8.4 \mathrm{~Hz}, 2 \mathrm{H}) ;{ }^{13} \mathrm{C}$ NMR $\left(75 \mathrm{MHz}, \mathrm{CDCl}_{3}\right) \delta 175.6,158.9,139.9,130.9$, $128.9,115.0,113.6,77.8,76.0,75.2,72.5,55.2,42.6,40.4,36.8,36.2,31.0,26.0,23.9$, 18.8, 18.3, 16.3, 15.2, 14.6, 9.6, -4.11, -4.14; HRMS calcd for $\mathrm{C}_{31} \mathrm{H}_{54} \mathrm{O}_{5} \mathrm{Si} 534.3741$, found 534.3734 .

(1R,2R)-1-ethyl-2-methylbut-3-enyl (2R,3S,4S,6R)-3-(tert-butyldimethylsilanyloxy)2,4,6-trimethyl-7-oxonon-8-enoate (21) 


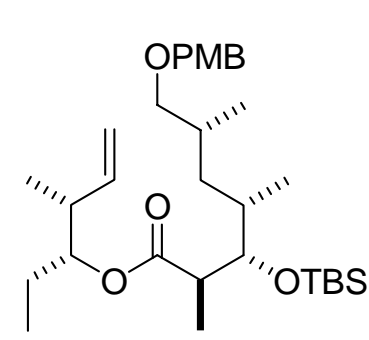

20

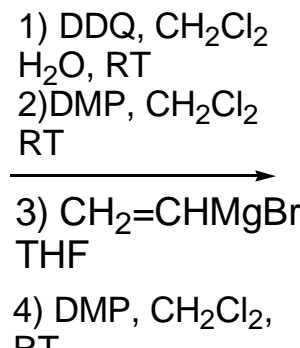

RT

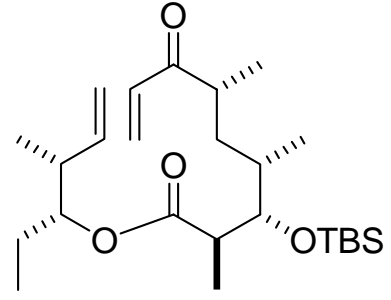

21

To a stirred solution of ester 20 (150 mg, $0.280 \mathrm{mmol})$ in $\mathrm{H}_{2} \mathrm{O}(0.3 \mathrm{~mL})$ and $\mathrm{CH}_{2} \mathrm{Cl}_{2}(3$ $\mathrm{mL})$ was added the DDQ (127 mg, $0.560 \mathrm{mmol})$ at room temperature. After $4 \mathrm{~h}$, the reaction mixture was diluted with $\mathrm{CH}_{2} \mathrm{Cl}_{2}(10 \mathrm{~mL})$, then to this mixture was added aqueous saturated $\mathrm{NaHCO}_{3}(5 \mathrm{~mL})$. The layers were then separated and the aqueous layer was extracted with $\mathrm{CH}_{2} \mathrm{Cl}_{2}(3 \times 5 \mathrm{~mL})$. The combined organic layers were washed with saturated aqueous $\mathrm{NaHCO}_{3}(10 \mathrm{~mL})$, water $(10 \mathrm{~mL})$, dried $\left(\mathrm{MgSO}_{4}\right)$, and concentrated. Purification of the residue by flash chromatography (hexane:EtOAc $=$ 10:1) afforded the desired primary alcohol $(112 \mathrm{mg}, 97 \%)$ as a colorless oil: $[\alpha]_{\mathrm{D}}^{24.1}$ $+32.1^{\circ}\left(c 1.23, \mathrm{CHCl}_{3}\right)$; IR (film) $v_{\max } 3456,2958,1724,1462,1381,1254,1180,1053$, 918, 837, $775 \mathrm{~cm}^{-1} ;{ }^{1} \mathrm{H}$ NMR $\left(300 \mathrm{MHz}, \mathrm{CDCl}_{3}\right) \delta 0.06(\mathrm{~s}, 3 \mathrm{H}), 0.07(\mathrm{~s}, 3 \mathrm{H}), 0.85(\mathrm{t}, J=$ $7.4 \mathrm{~Hz}, 3 \mathrm{H}), 0.89$ (s, 9H), $0.93(\mathrm{~d}, J=6.7 \mathrm{~Hz}, 3 \mathrm{H}), 0.95(\mathrm{~d}, J=6.6 \mathrm{~Hz}, 3 \mathrm{H}), 1.01(\mathrm{~d}, J=$ $6.8 \mathrm{~Hz}, 3 \mathrm{H}), 0.80-1.10(\mathrm{~m}, 1 \mathrm{H}), 1.16(\mathrm{~d}, J=7.0 \mathrm{~Hz}, 3 \mathrm{H}), 1.40-1.70(\mathrm{~m}, 5 \mathrm{H}), 1.81(\mathrm{~s}$, 1H), $2.45(\mathrm{~m}, 1 \mathrm{H}), 2.64(\mathrm{dddd}, J=7.1,7.1,7.1,7.1 \mathrm{~Hz}, 1 \mathrm{H}), 3.42(\mathrm{dd}, J=11.1,4.9, \mathrm{~Hz}$, $1 \mathrm{H}), 3.43(\mathrm{dd}, J=11.0,5.4 \mathrm{~Hz}, 1 \mathrm{H}), 3.88(\mathrm{dd}, J=7.3,2.5 \mathrm{~Hz}, 1 \mathrm{H}), 4.74$ (ddd, $J=7.6$, 6.0, 4.6 Hz, 1H), $5.03(\mathrm{~d}, J=9.6 \mathrm{~Hz}, 1 \mathrm{H}), 5.04(\mathrm{~d}, J=17.3 \mathrm{~Hz}, 1 \mathrm{H}), 5.74(\mathrm{ddd}, J=17.4$, 
10.4, 7.4 Hz, 1H); ${ }^{13} \mathrm{C}$ NMR $\left(75 \mathrm{MHz}, \mathrm{CDCl}_{3}\right) \delta 176.0,139.8,115.1,78.1,76.6,66.8$, $43.6,40.5,35.9,34.7,32.8,26.0,23.9,18.3,18.2,17.0,15.2,15.1,9.6,-3.95,-3.98$; HRMS calcd for $\mathrm{C}_{23} \mathrm{H}_{46} \mathrm{O}_{4} \mathrm{Si}$ 414.3165, found 414.3158.

To a solution of the alcohol $(87.5 \mathrm{mg}, 0.211 \mathrm{mmol})$ obtained as described in the previous procedure and $\mathrm{CH}_{2} \mathrm{Cl}_{2}(3 \mathrm{~mL})$ was added Dess-Martin periodinane $(179 \mathrm{mg}$, $0.422 \mathrm{mmol}$ ) at $0{ }^{\circ} \mathrm{C}$. The resulting solution was stirred for $2 \mathrm{~h}$ and was diluted with $\mathrm{CH}_{2} \mathrm{Cl}_{2}(2 \mathrm{~mL})$. After the reaction was completed, aqueous saturated $\mathrm{NaHCO}_{3}(10 \mathrm{~mL})$ and aqueous saturated $\mathrm{Na}_{2} \mathrm{~S}_{2} \mathrm{O}_{3}(5 \mathrm{~mL})$ were added. The resulting mixture was stirred and the organic layer was extracted and washed with saturated aqueous $\mathrm{NaHCO}_{3}(10$ $\mathrm{mL})$, water $(10 \mathrm{~mL})$, dried $\left(\mathrm{MgSO}_{4}\right)$, and concentrated. Purification of the residue by flash chromatography (hexane:EtOAc $=10: 1)$ afforded the desired aldehyde $(81 \mathrm{mg}$, $93 \%):[\alpha]_{\mathrm{D}}{ }^{24.7}+32.2^{\circ}\left(c 0.60, \mathrm{CHCl}_{3}\right) ;{ }^{1} \mathrm{H}$ NMR $\left(300 \mathrm{MHz}, \mathrm{CDCl}_{3}\right) \delta 0.05(\mathrm{~s}, 3 \mathrm{H}), 0.06$ (s, 3H), $0.85(\mathrm{t}, J=7.5 \mathrm{~Hz}, 3 \mathrm{H}), 0.89(\mathrm{~s}, 9 \mathrm{H}), 0.93(\mathrm{~d}, J=6.8 \mathrm{~Hz}, 3 \mathrm{H}), 1.00$ (d, $J=6.8$ $\mathrm{Hz}, 3 \mathrm{H}), 1.08(\mathrm{~d}, J=6.9 \mathrm{~Hz}, 3 \mathrm{H}), 1.10-1.20(\mathrm{~m}, 1 \mathrm{H}), 1.16(\mathrm{~d}, J=7.1 \mathrm{~Hz}, 3 \mathrm{H}), 1.40-1.70$ (m, 3H), 1.84 (ddd, $J=13.6,9.0,4.2 \mathrm{~Hz}, 1 \mathrm{H}), 2.35-2.50(\mathrm{~m}, 2 \mathrm{H}), 2.60$ (dddd, $J=7.0$, 7.0, 7.0, 7.0 Hz, 1H), $3.86(\mathrm{dd}, J=7.0,3.1 \mathrm{~Hz}, 1 \mathrm{H}), 4.73(\mathrm{ddd}, J=7.5,5.7,5.7 \mathrm{~Hz}, 1 \mathrm{H})$, $5.02(\mathrm{~d}, J=10.1 \mathrm{~Hz}, 1 \mathrm{H}), 5.04(\mathrm{~d}, J=18.5 \mathrm{~Hz}, 1 \mathrm{H}), 5.74(\mathrm{ddd}, J=17.4,10.4,7.4 \mathrm{~Hz}$, 
$1 \mathrm{H}) ;{ }^{13} \mathrm{C} \mathrm{NMR}\left(75 \mathrm{MHz}, \mathrm{CDCl}_{3}\right) \delta 205.0,175.3,139.8,115.1,78.0,76.1,44.0,43.2$, $40.4,36.5,32.9,26.0,23.9,18.3,16.3,15.1,14.9,14.5,9.6,-4.01,-4.07$.

To a stirred solution of the aldehyde $(61.0 \mathrm{mg}, 0.148 \mathrm{mmol})$ prepared as described in the previous procedure and THF $(1.5 \mathrm{ml})$ was added vinylmagnesium bromide $(1 \mathrm{M}$ in THF, $180 \mu \mathrm{L}, 0.180 \mathrm{mmol}$ ) at $0{ }^{\circ} \mathrm{C}$. After $20 \mathrm{~min}$, the reaction mixture was diluted by adding $\mathrm{Et}_{2} \mathrm{O}(5 \mathrm{~mL})$, and then to this was added a saturated aqueous $\mathrm{NH}_{4} \mathrm{Cl}$ solution $(5 \mathrm{~mL})$. The organic layer was separated, and the aqueous layer was extracted with ether $(3 \times 5$ $\mathrm{mL})$. The organic solutions were combined, dried $\left(\mathrm{MgSO}_{4}\right)$, and concentrated. Purification of the residue by flash chromatography (hexane:EtOAc $=9: 1$ ) afforded a vinyl alcohol (49.2 mg, 76\%) as a colorless oil.

To a stirred solution of the alcohol $(47.5 \mathrm{mg}, 0.108 \mathrm{mmol})$ prepared as described in the previous procedure in $\mathrm{CH}_{2} \mathrm{Cl}_{2}(1.5 \mathrm{~mL})$ was added Dess-Martin periodinane (91.6 mg, $0.216 \mathrm{mmol}$ ) at $0{ }^{\circ} \mathrm{C}$. The resulting solution was stirred for $2 \mathrm{~h}$ and diluted with $\mathrm{CH}_{2} \mathrm{Cl}_{2}$ $(15 \mathrm{~mL})$. After the reaction was completed, aqueous saturated $\mathrm{NaHCO}_{3}(10 \mathrm{~mL})$ and aqueous saturated $\mathrm{Na}_{2} \mathrm{~S}_{2} \mathrm{O}_{3}(5 \mathrm{~mL})$ were added. The resulting mixture was stirred and the organic layer was separated, washed with saturated aqueous $\mathrm{NaHCO}_{3}(5 \mathrm{~mL})$ and 
water $(5 \mathrm{~mL})$, dried $\left(\mathrm{MgSO}_{4}\right)$, and concentrated. Purification of the residue by flash chromatography (hexane:EtOAc $=10: 1)$ afforded the desired vinylketone 21 (44.8 mg, 95\%) as a colorless oil: $[\alpha]_{\mathrm{D}}^{24.4}+23.9^{\circ}\left(c\right.$ 2.00, $\left.\mathrm{CHCl}_{3}\right)$; IR (film) $v_{\max } 3082,2962,1728$, 1612, 1462, 1381, 1254, 1176, 1057, 914, 837, 775, $679 \mathrm{~cm}^{-1} ;{ }^{1} \mathrm{H}$ NMR (300 MHz, $\left.\mathrm{CDCl}_{3}\right) \delta 0.04(\mathrm{~s}, 6 \mathrm{H}), 0.85(\mathrm{t}, J=7.4 \mathrm{~Hz}, 3 \mathrm{H}), 0.88(\mathrm{~s}, 9 \mathrm{H}), 0.91(\mathrm{~d}, J=6.9 \mathrm{~Hz}, 3 \mathrm{H})$, $1.00(\mathrm{~d}, J=6.8 \mathrm{~Hz}, 3 \mathrm{H}), 1.09(\mathrm{~d}, J=6.9 \mathrm{~Hz}, 3 \mathrm{H}), 1.05-1.20(\mathrm{~m}, 1 \mathrm{H}), 1.16(\mathrm{~d}, J=7.1 \mathrm{~Hz}$, 3H), 1.45-1.68 (m, 3H), 1.84 (ddd, $J=13.6,9.0,4.2 \mathrm{~Hz}, 1 \mathrm{H}), 2.44(\mathrm{~m}, 1 \mathrm{H}), 2.59$ (dddd, $J=7.0,7.0,7.0,7.0 \mathrm{~Hz}, 1 \mathrm{H}), 2.80-3.00(\mathrm{~m}, 1 \mathrm{H}), 3.88(\mathrm{dd}, J=6.7,3.0 \mathrm{~Hz}, 1 \mathrm{H}) 4.72(\mathrm{ddd}$, $J=7.5,5.9,4.7 \mathrm{~Hz}, 1 \mathrm{H}), 5.02(\mathrm{~d}, J=10.1 \mathrm{~Hz}, 1 \mathrm{H}), 5.04(\mathrm{~d}, J=17.3 \mathrm{~Hz}, 1 \mathrm{H}), 5.74(\mathrm{ddd}$, $J=17.4,11.7,7.4 \mathrm{~Hz}, 1 \mathrm{H}), 5.75(\mathrm{dd}, J=10.4,1.5 \mathrm{~Hz} 1 \mathrm{H}), 6.26(\mathrm{dd}, J=17.4,1.5 \mathrm{~Hz}$, 1H), $6.44(\mathrm{dd}, J=17.4,10.3 \mathrm{~Hz}, 1 \mathrm{H}) ;{ }^{13} \mathrm{C} \mathrm{NMR}\left(75 \mathrm{MHz}, \mathrm{CDCl}_{3}\right) \delta 203.7,175.5,139.9$, $134.9,128.0,115.0,78.0,75.8,42.7,41.3,40.4,37.1,35.2,26.0,23.9,18.2,17.7,15.9$, 15.2, 14.8, 9.6, -4.0; HRMS calcd for $\mathrm{C}_{25} \mathrm{H}_{46} \mathrm{O}_{4} \mathrm{Si} 438.3165$, found 438.3164.

(2S,4R)-5-(tert-Butyldimethylsilanyloxy)-2,4-dimethylpentan-1-ol (10c)<smiles>CC(=O)OC[C@H](C)C[C@@H](C)CO</smiles>

$10 \mathrm{~b}$
1) TBSOTf, 2,6Lutidine

2) $\mathrm{KOH} \mathrm{CH} \mathrm{CH}_{3} \mathrm{OH}$, $\mathrm{H}_{2} \mathrm{O}$ RT

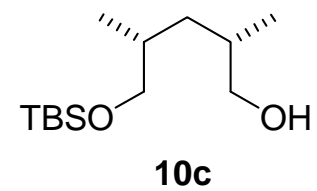


To a solution of alcohol 10b $(2.09 \mathrm{~g}, 12.0 \mathrm{mmol}), 2,6$-lutidine $(2.5 \mathrm{~mL}, 21.6 \mathrm{mmol})$ in $\mathrm{CH}_{2} \mathrm{Cl}_{2}(20 \mathrm{~mL})$ was added TBDMSOTf $(3.53 \mathrm{~mL}, 15.6 \mathrm{mmol})$ at $0{ }^{\circ} \mathrm{C}$. The reaction mixture was stirred for $4 \mathrm{~h}$ at $0{ }^{\circ} \mathrm{C}$. After reaction was completed, aqueous saturated $\mathrm{NaCl}(20 \mathrm{~mL})$ and aqueous saturated $\mathrm{NH}_{4} \mathrm{Cl}$ solution $(20 \mathrm{~mL})$ were added. The organic layer was separated and the aqueous layer was extracted with $\mathrm{CH}_{2} \mathrm{Cl}_{2}(3 \times 20 \mathrm{~mL})$. The organic solutions were combined, dried $\left(\mathrm{MgSO}_{4}\right)$, and concentrated. Purification of the residue by flash chromatography (hexane:EtOAc $=7: 1$ ) afforded the TBS-protected acetate $(3.43 \mathrm{~g}, 99 \%)$ as a colorless oil: $[\alpha]_{\mathrm{D}}^{23.1}+5.21^{\circ}\left(c 1.16, \mathrm{CHCl}_{3}\right)$; IR (film) $v_{\max }$ 2854, 1743, 1466, 1373, 1246, 1095, 1038, 841, $775 \mathrm{~cm}^{-1} ;{ }^{1} \mathrm{H}$ NMR (300 MHz, $\left.\mathrm{CDCl}_{3}\right)$ $\delta 0.02(\mathrm{~s}, 6 \mathrm{H}), 0.88(\mathrm{~s}, 9 \mathrm{H}), 0.92(\mathrm{~m}, 6 \mathrm{H}), 0.93(\mathrm{~m}, 1 \mathrm{H}), 1.46(\mathrm{ddd}, J=13.6,6.8,6.8$ Hz, 1H), 1.68 (dddd, $J=13.0,6.5,6.5,6.5 \mathrm{~Hz}, 1 \mathrm{H}$ ), 1.88 (ddd, $J=13.6,6.8,6.8,6.8 \mathrm{~Hz}$, $1 \mathrm{H}$ ), 2.04 (s, 3H), 3.31 (dddd, $J=9.8,9.8,9.8,5.5 \mathrm{~Hz}, 2 \mathrm{H}$ ), 3.97 3.77 (dddd, $J=10.7$, 10.7, 10.7, 5.4, Hz, 2H ); ${ }^{13} \mathrm{C} \mathrm{NMR}\left(75 \mathrm{MHz}, \mathrm{CDCl}_{3}\right) \delta 171.3,69.3,68.0,37.3,33.0$, 29.9, 25.9, 20.9, 18.3, 17.8, 17.5, -5.4; HRMS calcd for $\mathrm{C}_{15} \mathrm{H}_{32} \mathrm{O}_{3} \mathrm{Si} 288.2121$, found 288.2105 .

To a solution of the acetate $(3.43 \mathrm{~g}, 11.9 \mathrm{mmol})$ prepared in the previous step in $\mathrm{CH}_{3} \mathrm{OH}$ $(30 \mathrm{~mL})$ was added a solution of $\mathrm{KOH}(1.34 \mathrm{~g}, 23.8 \mathrm{mmol})$ in water $(4 \mathrm{~mL})$ dropwise at 
room temperature and the solution was stirred for $30 \mathrm{~min}$ at room temperature. The reaction was terminated by the addition of aqueous saturated $\mathrm{NH}_{4} \mathrm{Cl}(50 \mathrm{~mL})$. The mixture was extracted with ether $(3 \times 20 \mathrm{~mL})$. The organic layer was dried $\left(\mathrm{MgSO}_{4}\right)$, filtered, and concentrated. Purification by flash chromatography (hexane:EtOAc $=3: 1$ ) afforded the desired alcohol 10c $(2.58 \mathrm{~g}, 88 \%)$ as a colorless oil: $[\alpha]_{\mathrm{D}}^{16.1}-1.32^{\circ}(c 1.99$, $\mathrm{CHCl}_{3}$ ); IR (film) $v_{\max } 3344,2950,1466,1385,1254,1092,845,775,667 \mathrm{~cm}^{-1} ;{ }^{1} \mathrm{H}$ NMR (300 MHz, $\left.\mathrm{CDCl}_{3}\right) \delta 0.04(\mathrm{~s}, 6 \mathrm{H}), 0.89(\mathrm{~s}, 9 \mathrm{H}), 0.93(\mathrm{~m}, 7 \mathrm{H}), 1.43(\mathrm{~m}, 1 \mathrm{H}), 1.58$ (s, 1H), 1.71 (dddd, $J=13.2,13.2,6.7,6.7 \mathrm{~Hz}, 2 \mathrm{H}), 3.53 \sim 3.34(\mathrm{~m}, 4 \mathrm{H}) ;{ }^{13} \mathrm{C}$ NMR $(75$ $\left.\mathrm{MHz}, \mathrm{CDCl}_{3}\right) \delta 68.2,37.3,33.2,25.9,18.3,17.8,17.7,-5.4$; HRMS calcd for $\mathrm{C}_{13} \mathrm{H}_{30} \mathrm{O}_{2} \mathrm{Si}$ 246.2015, found 246.2025.

(2S,4R)-5-(tert-Butyldimethylsilanyloxy)-2,4-dimethylpentanal (11b)

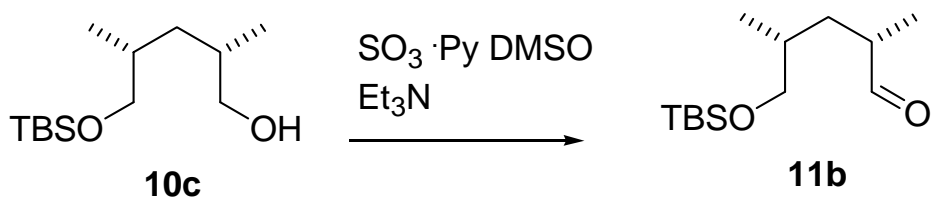

To a solution of the alcohol 10c $(3.20 \mathrm{~g}, 13.0 \mathrm{mmol})$ and triethylamine $(18.1 \mathrm{~mL}, 130$ mmol) in $\mathrm{CH}_{2} \mathrm{Cl}_{2}(30 \mathrm{~mL})$, was added a solution of pyridine- $\mathrm{SO}_{3}$ complex $(7.79 \mathrm{~g}, 45.5$ mmol) in DMSO $(20 \mathrm{ml})$ at room temperature. The mixture was stirred for $1 \mathrm{~h}$ at room temperature. The reaction was terminated by addition of aqueous saturated $\mathrm{NaCl}(30$ 
$\mathrm{mL})$. The mixture was extracted with $\mathrm{CH}_{2} \mathrm{Cl}_{2}(3 \times 30 \mathrm{~mL})$ and the organic layer was separated, dried $\left(\mathrm{MgSO}_{4}\right)$, and concentrated. Purification of the residue by flash chromatography (hexane:EtOAc $=2: 1$ ) afforded the desired aldehyde 11b $(2.75 \mathrm{~g}$, 87\%) as a colorless oil: ${ }^{1} \mathrm{H}$ NMR $\left(300 \mathrm{MHz}, \mathrm{CDCl}_{3}\right) \delta 0.03(\mathrm{~s}, 6 \mathrm{H}), 0.88(\mathrm{~s}, 9 \mathrm{H}), 0.89$ (d, $J=6.8 \mathrm{~Hz}, 3 \mathrm{H}), 1.09(\mathrm{~d}, J=7.0 \mathrm{~Hz}, 3 \mathrm{H}), 1.11(\mathrm{~m}, 1 \mathrm{H}), 1.67$ (dddd, $J=12.9,6.4$, 6.4, $6.4 \mathrm{~Hz}, 1 \mathrm{H}), 1.86(\mathrm{~m}, 1 \mathrm{H}) 2.46$ (ddddd, $J=14.3,7.0,7.0,7.0,2.3 \mathrm{~Hz}, 1 \mathrm{H}) 3.42$ (d, $J=5.7 \mathrm{~Hz}, 2 \mathrm{H}), 9.57(\mathrm{~d}, J=2.3 \mathrm{~Hz}, 1 \mathrm{H}) ;{ }^{13} \mathrm{C} \mathrm{NMR}\left(75 \mathrm{MHz}, \mathrm{CDCl}_{3}\right) \delta 205.28,67.8$, $44.2,34.5,33.4,25.9,28.3,17.1,16.5,14.3,-5.5$.

(R)-4-Benzyl-3-[(2R,3S,4S,6R)-7-(tert-butyldimethylsilanyloxy)-3-(4methoxybenzyloxy)-2,4,6-trimethylheptanoyl]oxazolidin-2-one (15)

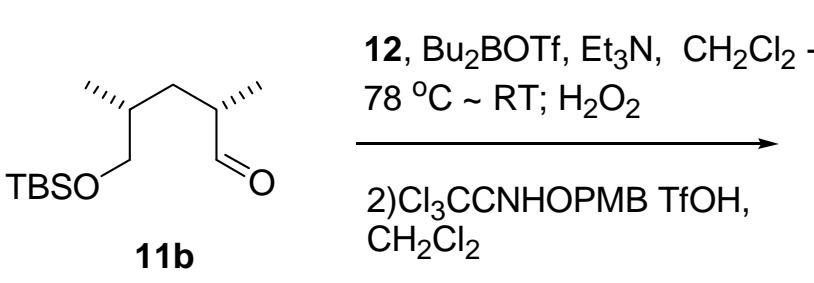<smiles>[R16]O[C@H]([C@@H](C)C[C@H](C)C[SeH])[C@@H](C)C([X])=O</smiles>

15

To a stirred solution of the acylated oxazolidinone $12(2.67 \mathrm{~g}, 11.4 \mathrm{mmol})$ in $\mathrm{CH}_{2} \mathrm{Cl}_{2}$ $(15 \mathrm{~mL})$ at $0{ }^{\circ} \mathrm{C}$, was added dibutylboron triflate solution $(12.5 \mathrm{~mL}$ of a $1.0 \mathrm{M}$ solution in $\left.\mathrm{CH}_{2} \mathrm{Cl}_{2}, 12.5 \mathrm{mmol}\right)$ and triethylamine $(2.0 \mathrm{~mL}, 14.8 \mathrm{mmol})$ dropwise at $0{ }^{\circ} \mathrm{C}$. The solution was cooled down to $-78^{\circ} \mathrm{C}$. To this solution was added a solution of aldehyde 
$11 \mathrm{~b}(2.55 \mathrm{~g}, 10.4 \mathrm{mmol})$ prepared in the previous procedure in $\mathrm{CH}_{2} \mathrm{Cl}_{2}(4 \mathrm{~mL})$ at $-78{ }^{\circ} \mathrm{C}$. The resulting solution was stirred for $20 \mathrm{~min}$ at $-78^{\circ} \mathrm{C}$. The solution was warmed to $0{ }^{\circ} \mathrm{C}$ and stirred for $1 \mathrm{~h}$ additionally. The reaction was terminated by adding a $\mathrm{pH} 7$ aqueous phosphate buffer solution (0.2 M-sodium hydrogen phosphate:0.1 M-citric acid $=82: 18,10 \mathrm{~mL})$ and methanol $(30 \mathrm{~mL})$. To this cloudy solution was added a solution of methanol and $30 \%$ hydrogen peroxide $(2: 1,30 \mathrm{~mL})$ slowly and the resulting solution was stirred for $1 \mathrm{~h}$ at $0{ }^{\circ} \mathrm{C}$. After the solution was concentrated, it was extracted with diethyl ether $(3 \times 20 \mathrm{~mL})$. The combined organic extracts were washed with $5 \%$ aqueous sodium bicarbonate $(20 \mathrm{~mL})$ and aqueous saturated $\mathrm{NaCl}(25 \mathrm{~mL})$, dried $\left(\mathrm{MgSO}_{4}\right)$, and concentrated. Purification of the residue by flash chromatography (hexane:EtOAc $=4: 1)$ afforded the desired aldol product $(3.97 \mathrm{~g}, 80 \%)$ as a white solid: $\operatorname{mp} 74 \sim 76^{\circ} \mathrm{C} ;[\alpha]_{\mathrm{D}}{ }^{25.1}-39.7^{\circ}\left(c 1.42, \mathrm{CHCl}_{3}\right)$; IR (film) $v_{\max } 3529,2951,1782,1689$, 1462, 1385, 1211, 1092, 980, 841, 771, $702 \mathrm{~cm}^{-1} ;{ }^{1} \mathrm{H}$ NMR $\left(300 \mathrm{MHz}, \mathrm{CDCl}_{3}\right) \delta 0.04(\mathrm{~s}$, 6H), $0.89(\mathrm{~s}, 9 \mathrm{H}), 0.93(\mathrm{~m}, 6 \mathrm{H}), 1.22(\mathrm{~d}, J=7.0 \mathrm{~Hz}, 3 \mathrm{H}), 1.23(\mathrm{~m}, 1 \mathrm{H}), 1.71(\mathrm{~m}, 3 \mathrm{H})$, $2.79(\mathrm{~m}, 1 \mathrm{H}), 2.89(\mathrm{~s}, 1 \mathrm{H}), 3.31(\mathrm{~m}, 2 \mathrm{H}), 3.54(\mathrm{~m}, 2 \mathrm{H}), 3.96(\mathrm{dddd}, J=6.8,6.8,6.8,2.1$ $\mathrm{Hz}, 1 \mathrm{H}), 4.20(\mathrm{~m}, 2 \mathrm{H}), 4.69$ (ddddd, J=10.0, 3.1, 3.1, 3.1, 3.1 Hz, 1H), 7.37 7.20 (m, 5H); ${ }^{13} \mathrm{C}$ NMR $\left(75 \mathrm{MHz}, \mathrm{CDCl}_{3}\right) \delta 177.6,152.9,135.1,129.4,128.9,127.4,76.0,68.1$, $66.1,55.3,39.7,37.9,37.7,34.0,33.4,25.9,18.9,18.3,16.2,9.5,-5.4$; HRMS calcd for 
$\mathrm{C}_{26} \mathrm{H}_{43} \mathrm{NO}_{3} \mathrm{Si}$ 477.2911, found 477.2911.

To a stirred solution of aldol adduct (4.33 $\mathrm{g}, 9.06 \mathrm{mmol})$ prepared in the previous procedure, 4-methoxybenzyl 2,2,2-trichloroacetimidate $(6.41 \mathrm{~g}, 22.7 \mathrm{mmol})$ in $\mathrm{CH}_{2} \mathrm{Cl}_{2}$ (30 mL) was added a solution of trifluoromethanesulfonic acid (10 drops) in ether (10 $\mathrm{mL})$ dropwise via a cannula at $0{ }^{\circ} \mathrm{C}$. After $30 \mathrm{~min}$ aqueous saturated $\mathrm{NaHCO}_{3}(30 \mathrm{~mL})$ was added and the mixture was extracted with $\mathrm{CH}_{2} \mathrm{Cl}_{2}(3 \times 20 \mathrm{~mL})$. The organic phase was washed with $\mathrm{H}_{2} \mathrm{O}(20 \mathrm{~mL})$, dried $\left(\mathrm{MgSO}_{4}\right)$ and concentrated. Purification by flash chromatography (hexane:EtOAc $=7: 1)$ provided the PMB protected product $15(3.87 \mathrm{~g}$, $71 \%$ ) as a colorless oil: $[\alpha]_{\mathrm{D}}^{25.0}-22.2^{\circ}\left(c 1.05, \mathrm{CHCl}_{3}\right)$; IR (film) $v_{\max } 2951,1782,1697$, 1612, 1508, 1462, 1381, 1246, 1092, 968, 833, 771, $702 \mathrm{~cm}^{-1} ;{ }^{1} \mathrm{H}$ NMR (300 MHz, $\left.\mathrm{CDCl}_{3}\right) \delta 0.00(\mathrm{~s}, 6 \mathrm{H}), 0.88(\mathrm{~m}, 12 \mathrm{H}), 0.98(\mathrm{~d}, J=6.7 \mathrm{~Hz}, 3 \mathrm{H}), 1.28(\mathrm{~d}, J=6.9 \mathrm{~Hz}, 3 \mathrm{H})$ $1.36(\mathrm{~m}, 1 \mathrm{H}), 1.51(\mathrm{~m}, 1 \mathrm{H}), 1.73(\mathrm{~m}, 2 \mathrm{H}), 2.74(\mathrm{dd}, J=13.1,9.7 \mathrm{~Hz}, 1 \mathrm{H}), 3.24(\mathrm{~m}, 2 \mathrm{H})$, $3.47(\mathrm{dd}, J=9.7,4.6 \mathrm{~Hz}, 1 \mathrm{H}), 3.58(\mathrm{t}, J=5.5 \mathrm{~Hz}, 1 \mathrm{H}), 3.76(\mathrm{~s}, 3 \mathrm{H}), 3.99(\mathrm{~m}, 1 \mathrm{H}), 4.12$ $(\mathrm{m}, 2 \mathrm{H}), 4.47(\mathrm{~m}, 3 \mathrm{H}), 6.85(\mathrm{~d}, J=8.4 \mathrm{~Hz}, 2 \mathrm{H}), 7.19(\mathrm{~d}, J=6.9 \mathrm{~Hz}, 2 \mathrm{H}), 7.29(\mathrm{~m}, 5 \mathrm{H})$; ${ }^{13} \mathrm{C}$ NMR $\left(75 \mathrm{MHz}, \mathrm{CDCl}_{3}\right) \delta 175.9,159.0,152.8,135.2,130.7,129.4,128.9,127.3$, $113.6,84.11,74.2,67.8,67.5,55.6,55.1,40.5,37.6,36.7,34.6,33.3,25.9,18.8,18.7$, 18.2, 12.5, -5.5; HRMS calcd for $\mathrm{C}_{34} \mathrm{H}_{51} \mathrm{NO}_{6} \mathrm{Si}[\mathrm{M}+\mathrm{Na}]^{+}$620.3383, found 620.3386. 


\section{(2R,3S,4S,6R)-7-(tert-butyldimethylsilanyloxy)-3-(4-methoxybenzyloxy)-2,4,6-}

\section{trimethyl-heptanal (23)}

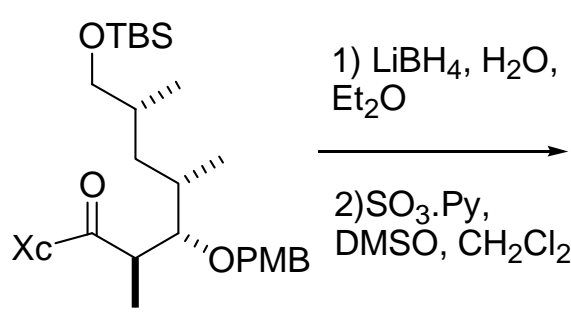

15<smiles>[R16]O[C@@H]([C@@H](C)C=O)[C@H](C)C[C@@H](C)C[SeH-]</smiles>

23

To a solution of $\mathbf{1 5}(3.87 \mathrm{~g}, 6.47 \mathrm{mmol})$ in ether $(30 \mathrm{~mL})$ was added distilled water $(175$ $\mu \mathrm{L}, 9.71 \mathrm{mmol})$ at room temperature. After the solution was cooled to $0{ }^{\circ} \mathrm{C}$, lithium borohydride $(6.50 \mathrm{~mL}$ of a $2.0 \mathrm{M}$ solution in THF, $13.0 \mathrm{mmol})$ was added slowly with stirring. After $10 \mathrm{~min}$, the temperature of the solution was raised to room temperature, and stirred for additional $2 \mathrm{~h}$. The reaction was terminated with addition of an aqueous $\mathrm{NaOH}$ solution $(1.0 \mathrm{M}, 20 \mathrm{~mL})$ and extracted with ether $(3 \times 30 \mathrm{~mL})$. After the organic layer was washed with aqueous saturated $\mathrm{NaCl}(30 \mathrm{~mL})$, the ethereal solution was dried $\left(\mathrm{MgSO}_{4}\right)$ and concentrated. Purification by flash chromatography (hexane:EtOAc $\left.=3: 1\right)$ provided the desired alcohol as a colorless liquid $(1.85 \mathrm{~g}, 67 \%):[\alpha]_{\mathrm{D}}^{23.0}+7.79^{\circ}(c 1.06$, $\mathrm{CHCl}_{3}$ ); IR (film) $v_{\max } 3413,2951,1612,1512,1462,1385,1300,1250,1173,1088$, 837, 775, $667 \mathrm{~cm}^{-1} ;{ }^{1} \mathrm{H}$ NMR $\left(300 \mathrm{MHz}, \mathrm{CDCl}_{3}\right) \delta 0.18(\mathrm{~s}, 6 \mathrm{H}), 1.04(\mathrm{~s}, 9 \mathrm{H}), 1.07(\mathrm{~m}$, $6 \mathrm{H}), 1.11(\mathrm{~d}, J=2.8 \mathrm{~Hz}, 3 \mathrm{H}), 1.83(\mathrm{~m}, 2 \mathrm{H}), 2.08(\mathrm{~m}, 3 \mathrm{H}), 3.49(\mathrm{~m}, 2 \mathrm{H}), 3.63(\mathrm{q}, J=9.8$, 
$5.1 \mathrm{~Hz}, 1 \mathrm{H}), 3.72(\mathrm{~d}, J=6.0 \mathrm{~Hz}, 1 \mathrm{H}), 3.95(\mathrm{~s}, 3 \mathrm{H}), 4.66(\mathrm{q}, J=38.4,11.0 \mathrm{~Hz}, 2 \mathrm{H}), 7.02$

$(\mathrm{d}, J=8.6 \mathrm{~Hz}, 2 \mathrm{H}), 7.43(\mathrm{~d}, J=8.5 \mathrm{~Hz}, 2 \mathrm{H}) ;{ }^{13} \mathrm{C} \mathrm{NMR}\left(75 \mathrm{MHz}, \mathrm{CDCl}_{3}\right) \delta 159.0$, $131.0,129.2,113.7,84.1,73.3,67.8,66.9,55.23,37.1,33.1,32.9,25.9,18.5,18.3,16.9$, 11.6, -5.4; HRMS calcd for $\mathrm{C}_{24} \mathrm{H}_{44} \mathrm{NO}_{4} \mathrm{Si} 424.3009$, found 424.3040.

To a solution of the alcohol $(1.40 \mathrm{~g}, 3.30 \mathrm{mmol})$ prepared as described in the previous procedure and triethylamine $(2.30 \mathrm{~mL}, 16.5 \mathrm{mmol})$ in $\mathrm{CH}_{2} \mathrm{Cl}_{2}(30 \mathrm{~mL})$ was added a solution of pyridine-SO $\mathrm{SO}_{3}$ complex $(1.98 \mathrm{~g}, 11.6 \mathrm{mmol})$ in DMSO $(10 \mathrm{~mL})$ under room temperature with stirring. After $1 \mathrm{~h}$, the reaction mixture was terminated by addition of aqueous saturated $\mathrm{NaCl}(20 \mathrm{~mL})$. The mixture was extracted with $\mathrm{CH}_{2} \mathrm{Cl}_{2}(3 \times 20 \mathrm{~mL})$ and the organic solution was dried $\left(\mathrm{MgSO}_{4}\right)$ and concentrated. Purifition of the residue by flash chromatography (hexane:EtOAc $=7: 1)$ afforded the desired aldehyde $23(1.30$ g, 93\%) as a colorless liquid: ${ }^{1} \mathrm{H}$ NMR $\left(300 \mathrm{MHz}, \mathrm{CDCl}_{3}\right) \delta 0.00(\mathrm{~s}, 6 \mathrm{H}), 0.85(\mathrm{~s}, 9 \mathrm{H})$, $0.92(\mathrm{~m}, 6 \mathrm{H}), 1.14(\mathrm{~d}, J=6.5 \mathrm{~Hz}, 3 \mathrm{H}) 1.69 \sim 1.52(\mathrm{~m}, 2 \mathrm{H}), 1.90(\mathrm{~m}, 1 \mathrm{H}), 2.55(\mathrm{~m}, 1 \mathrm{H})$, $3.46 \sim 3.27(\mathrm{~m}, 2 \mathrm{H}), 3.64(\mathrm{~m}, 1 \mathrm{H}), 3.77(\mathrm{~s}, 3 \mathrm{H}), 4.37$ (q, $J=10.5 \mathrm{~Hz}, 2 \mathrm{H}), 6.84(\mathrm{~d}, J=$ $7.5 \mathrm{~Hz}, 2 \mathrm{H}), 7.19(\mathrm{~d}, J=7.7 \mathrm{~Hz}, 2 \mathrm{H}), 9.71(\mathrm{~s}, 1 \mathrm{H}) ;{ }^{13} \mathrm{C} \mathrm{NMR}\left(75 \mathrm{MHz}, \mathrm{CDCl}_{3}\right) \delta 204.8$, $159.1,130.4,129.2,113.6,81.8,72.9,67.6,55.2,48.0,37.0,33.5,33.2,25.9,18.4,18.3$, $16.8,8.6,-5.4$. 
(R)-4-Benzyl-3-[(2R,3S,4S,5S,6S,8R)-9-(tert-butyldimethylsilanyloxy)-3-hydroxy-5-

(4-methoxybenzyloxy)-2,4,6,8-tetramethylnonanoyl]oxazolidin-2-one (24)<smiles>[R16]O[C@H]([C@@H](C)C=O)[C@H](C)C[C@H](C)CC(C)C[OH2+]</smiles>

23<smiles>CCC(=O)N1C(=O)OC[C@H]1Cc1ccccc1</smiles>

12<smiles>[Y16]O[C@H]([C@@H](C)C[C@H](C)C[OH2+])[C@@H](C)[C@H](O)[C@@H](C)C([X])=O</smiles>

24

To a stirred solution of the acylated oxazolidinone $12(718 \mathrm{mg}, 3.08 \mathrm{mmol})$ in $\mathrm{CH}_{2} \mathrm{Cl}_{2}$

$(10 \mathrm{~mL})$ was added $1 \mathrm{M}$ dibutylboron triflate solution $(4.0 \mathrm{~mL}, 4.0 \mathrm{mmol})$ and triethylamine $(645 \mu \mathrm{L}, 4.61 \mathrm{mmol})$ dropwise at $0{ }^{\circ} \mathrm{C}$. The solution was cooled down to $78{ }^{\circ} \mathrm{C}$. To this solution was added a solution of aldehyde $23(1.30 \mathrm{~g}, 3.08 \mathrm{mmol})$ prepared as described in the previous procedure in $\mathrm{CH}_{2} \mathrm{Cl}_{2}(2 \mathrm{~mL})$ at $-78{ }^{\circ} \mathrm{C}$. The resulting solution was stirred for $20 \mathrm{~min}$ at $-78^{\circ} \mathrm{C}$. The solution was warmed to $0{ }^{\circ} \mathrm{C}$ and stirred for $1 \mathrm{~h}$ additionally. The reaction was terminated by adding a $\mathrm{pH} 7$ aqueous phosphate buffer solution (0.2 M-sodium hydrogen phosphate:0.1 M-citric acid= 82:18, $10 \mathrm{~mL})$ and methanol $(20 \mathrm{~mL})$. To this cloudy solution was added a solution of methanol and 30\% Hydrogen peroxide $(2: 1,20 \mathrm{~mL})$ slowly and the resulting solution was stirred for $1 \mathrm{~h}$ at $0{ }^{\circ} \mathrm{C}$. After the solution was concentrated, it was extracted with 
diethyl ether $(3 \times 20 \mathrm{~mL})$. The combined organic extracts was washed with $5 \%$ aqueous sodium bicarbonate $(20 \mathrm{~mL})$ and aqueous saturated $\mathrm{NaCl}(20 \mathrm{~mL})$, dried $\left(\mathrm{MgSO}_{4}\right)$, and concentrated. Purification of the residue by flash chromatography (hexane:EtOAc $=5: 1$ ) afforded the desired aldol product $24(1.8 \mathrm{~g}, 82 \%)$ as a yellow liquid: $[\alpha]_{\mathrm{D}}^{24.3}-20.4^{\circ}(c$ 1.77, $\mathrm{CHCl}_{3}$ ); IR (film) $v_{\max } 3510,2951,1782,1693,1612,1512,1462,1385,1246$, 1092, 841, 771, $702 \mathrm{~cm}^{-1} ;{ }^{1} \mathrm{H}$ NMR $\left(300 \mathrm{MHz}, \mathrm{CDCl}_{3}\right) \delta 0.00(\mathrm{~s}, 6 \mathrm{H}), 0.85(\mathrm{~s}, 9 \mathrm{H})$, $0.91(\mathrm{~m}, 6 \mathrm{H}), 0.95(\mathrm{~d}, J=6.9 \mathrm{~Hz}, 3 \mathrm{H}), 1.25(\mathrm{~d}, J=6.8 \mathrm{~Hz}, 3 \mathrm{H}), 1.56(\mathrm{~m}, 1 \mathrm{H}), 1.73(\mathrm{~m}$, 2H), $1.97(\mathrm{~m}, 1 \mathrm{H}), 2.70(\mathrm{dd}, J=13.3,9.4 \mathrm{~Hz}, 1 \mathrm{H}), 3.14(\mathrm{~m}, 2 \mathrm{H}), 3.27(\mathrm{~m}, 1 \mathrm{H}), 3.33(\mathrm{~m}$, $1 \mathrm{H}), 3.48(\mathrm{dd}, J=9.7,5.0 \mathrm{~Hz}, 1 \mathrm{H}), 3.72(\mathrm{~s}, 3 \mathrm{H}), 3.83(\mathrm{~m}, 1 \mathrm{H}), 3.93(\mathrm{~m}, 1 \mathrm{H}), 4.11(\mathrm{~m}$, 2H), $4.35(\mathrm{~m}, 1 \mathrm{H}), 4.56(\mathrm{~m}, 2 \mathrm{H}), 6.79(\mathrm{~d}, J=8.6 \mathrm{~Hz}, 2 \mathrm{H}), 7.14(\mathrm{~d}, J=8.2 \mathrm{~Hz}, 2 \mathrm{H})$, 7.24(m, 5H); ${ }^{13} \mathrm{C}$ NMR (75 MHz, $\left.\mathrm{CDCl}_{3}\right) \delta 176.5,158.9,152.5,134.9,130.7,129.3$, $129.0,128.8,127.2,113.6,86.3,77.2,74.8,72.9,67.7,65.8,55.1,54.8,40.4,37.5,37.0$, 33.0, 33.0, 25.9, 18.4, 18.2, 16.6, 13.4, 8.7, -5.5; HRMS calcd for $\mathrm{C}_{37} \mathrm{H}_{57} \mathrm{NO}_{7} \mathrm{Si}[\mathrm{M}+$ $\mathrm{Na}]^{+}$678.3802, found 678.3805 .

(R)-4-Benzyl-3-[(2R,3S,4R,5S,6S,8R)-3,9-di(tert-butyldimethylsilanyloxy)-5-(4methoxybenzyloxy)-2,4,6,8-tetramethyl-3-nonanoyl]oxazolidin-2-one (25) 


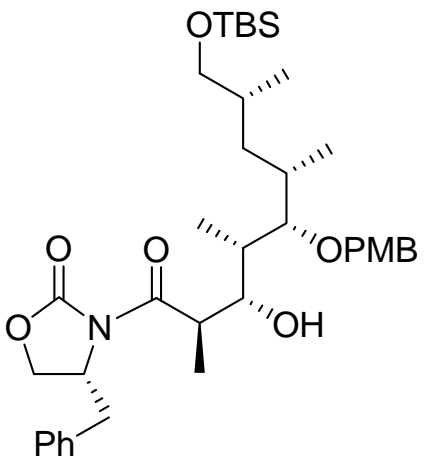

24

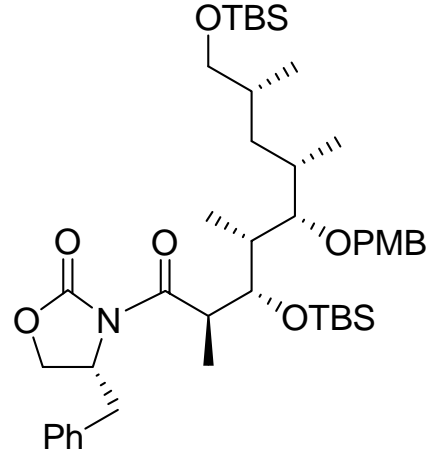

25

To a stirred solution of alcohol 24 (1.57 g, $2.40 \mathrm{mmol})$, 2,6-lutidine (503 $\mu \mathrm{L}, 4.32$ mmol) in $\mathrm{CH}_{2} \mathrm{Cl}_{2}(20 \mathrm{~mL})$ was added TBDMSOTf $(815 \mu \mathrm{L}, 3.60 \mathrm{mmol})$ at $0{ }^{\circ} \mathrm{C}$. The reaction mixture was stirred for $4 \mathrm{~h}$ at $0{ }^{\circ} \mathrm{C}$. After reaction was completed, aqueous saturated $\mathrm{NaCl}(20 \mathrm{~mL})$ and aqueous saturated $\mathrm{NH}_{4} \mathrm{Cl}$ solution $(10 \mathrm{~mL})$ were added. The organic layer was separated and the aqueous layer was extracted with $\mathrm{CH}_{2} \mathrm{Cl}_{2}(3 \times$ $20 \mathrm{~mL})$. The organic solutions were combined, dried $\left(\mathrm{MgSO}_{4}\right)$, and concentrated. Purification of the residue by flash chromatography (hexane/EtOAc $=5: 1$ ) afforded the TBS-protected product $25(1.53 \mathrm{~g}, 83 \%)$ as a colorless oil: $[\alpha]_{\mathrm{D}}^{24.2}-38.3^{\circ}(c$ 1.74, $\mathrm{CHCl}_{3}$ ); IR (film) $v_{\max } 2951,1782,1697,1612,1512,1462,1381,1250,1088,968,837$, 775, $698 \mathrm{~cm}^{-1} ;{ }^{1} \mathrm{H}$ NMR (300 MHz, $\left.\mathrm{CDCl}_{3}\right) \delta 0.00(\mathrm{~m}, 12 \mathrm{H}), 0.85(\mathrm{~s}, 9 \mathrm{H}), 0.89(\mathrm{~m}, 12 \mathrm{H})$, $0.99(\mathrm{~m}, 7 \mathrm{H}), 1.19(\mathrm{~d}, J=6.3 \mathrm{~Hz}, 3 \mathrm{H}), 1.25(\mathrm{~m}, 1 \mathrm{H}), 1.66(\mathrm{~m}, 2 \mathrm{H}), 1.92(\mathrm{~m}, 1 \mathrm{H}), 2.66$ $(\mathrm{dd}, J=13.2,9.6 \mathrm{~Hz}, 1 \mathrm{H}), 3.14(\mathrm{dd}, J=13.2,2.3 \mathrm{~Hz}, 2 \mathrm{H}), 3.30(\mathrm{~m}, 2 \mathrm{H}), 3.62(\mathrm{dd}, J=$ 9.8, 4.4 Hz, 1H), $3.70(\mathrm{~s}, 3 \mathrm{H}), 3.93(\mathrm{~m}, 4 \mathrm{H}), 4.42(\mathrm{~m}, 3 \mathrm{H}), 6.78(\mathrm{~d}, J=8.5 \mathrm{~Hz}, 2 \mathrm{H}), 7.11$ 
$(\mathrm{d}, J=6.9 \mathrm{~Hz}, 2 \mathrm{H}), 7.23(\mathrm{~m}, 5 \mathrm{H}) ;{ }^{13} \mathrm{C} \mathrm{NMR}\left(75 \mathrm{MHz}, \mathrm{CDCl}_{3}\right) \delta 175.5,158.8,152.7$, $135.2,131.4,129.4,128.9,128.9,127.3,113.6,83.8,74.1,73.5,67.8,65.7,55.4,55.2$, $41.7,40.8,37.5,35.3,33.1,32.5,26.2,26.0,25.6,19.1,18.5,18.4,17.4,15.4,11.2,-3.4$, $-4.0,-5.3,-5.3$; HRMS calcd for $\mathrm{C}_{43} \mathrm{H}_{71} \mathrm{NO}_{7} \mathrm{Si}_{2}[\mathrm{M}+\mathrm{Na}]^{+}$792.4667, found 792.4670.

(2R,3S,4R,5S,6S,8R)-3,9-di(tert-butyldimethylsilanyloxy)-5-(4-methoxybenzyloxy)-

2,4,6,8-tetramethylnonanoic acid (26)

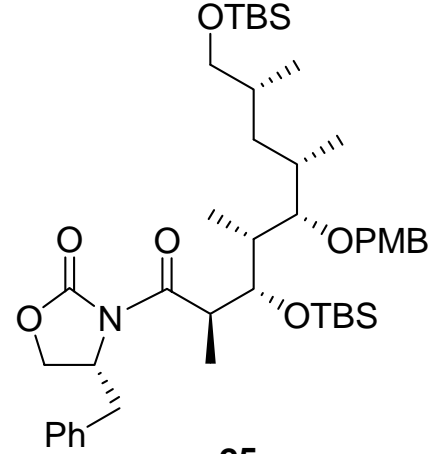

25

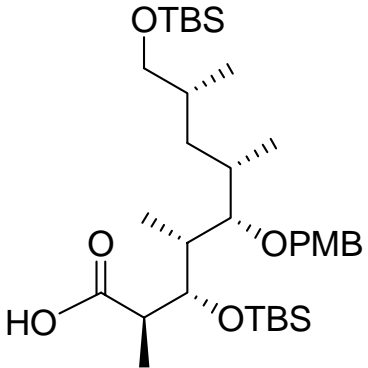

26

To a stirred solution of $25(1.53 \mathrm{~g}, 1.99 \mathrm{mmol})$ in tetrahydrofuran-water $(4: 1,15 \mathrm{~mL})$ was added $30 \%$ aqueous hydrogen peroxide $(956 \mu \mathrm{L}, 7.96 \mathrm{mmol})$ and an aqueous solution of lithium hydroxide $(125 \mathrm{mg}, 2.99 \mathrm{mmol})$ dissolved in $\mathrm{H}_{2} \mathrm{O}(2 \mathrm{~mL})$. After $5 \mathrm{~h}$, the reaction was terminated by adding aqueous saturated $\mathrm{Na}_{2} \mathrm{SO}_{3}(10 \mathrm{~mL})$ and aqueous saturated $\mathrm{NH}_{4} \mathrm{Cl}(10 \mathrm{~mL})$. The resulting mixture was extracted with ether $(3 \times 20 \mathrm{~mL})$. The organic layers was dried $\left(\mathrm{MgSO}_{4}\right)$ and concentrated. Purification of the residue by flash chromatography (hexane:EtOAc = 4:1) afforded carboxylic acid $26(987 \mathrm{mg}, 81 \%)$ 
as a colorless oil: $[\alpha]_{\mathrm{D}}^{19.1}-6.0^{\circ}\left(c 1.27, \mathrm{CHCl}_{3}\right)$; IR (film) $v_{\max } 2958,1705,1612,1512$, 1462, 1385, 1250, 1053, 949, 841, 775, $675 \mathrm{~cm}^{-1} ;{ }^{1} \mathrm{H}$ NMR $\left(300 \mathrm{MHz}, \mathrm{CDCl}_{3}\right) \delta 0.00$ (m, 12H), $0.87(\mathrm{~m}, 21 \mathrm{H}), 0.94(\mathrm{~m}, 7 \mathrm{H}), 1.12(\mathrm{~d}, J=6.8 \mathrm{~Hz}, 3 \mathrm{H}), 1.45(\mathrm{~m}, 1 \mathrm{H}), 1.67(\mathrm{~m}$, 1H), $1.85(\mathrm{~m}, 2 \mathrm{H}), 2.73(\mathrm{~m}, 1 \mathrm{H}), 3.13(\mathrm{~m}, 1 \mathrm{H}), 3.26(\mathrm{dd}, J=9.5,7.1 \mathrm{~Hz}, 1 \mathrm{H}), 3.51(\mathrm{dd}$, $J=9.7,4.4 \mathrm{~Hz}, 1 \mathrm{H}), 3.76(\mathrm{~s}, 3 \mathrm{H}), 4.07(\mathrm{~m}, 1 \mathrm{H}), 4.46(\mathrm{~m}, 2 \mathrm{H}), 6.82(\mathrm{~d}, J=8.5 \mathrm{~Hz}, 2 \mathrm{H})$, $7.25(\mathrm{~d}, J=8.6 \mathrm{~Hz}, 2 \mathrm{H}) ;{ }^{13} \mathrm{C} \mathrm{NMR}\left(75 \mathrm{MHz}, \mathrm{CDCl}_{3}\right) \delta 180.6,159.0,131.1,129.1$, $113.7,84.2,74.5,73.9,67.7,55.2,43.3,39.8,36.2,33.4,33.2,26.1,26.0,18.9,18.4$, 18.4, 17.2, 11.3, 11.0, -3.9, -4.1, -5.4; HRMS calcd for $\mathrm{C}_{33} \mathrm{H}_{62} \mathrm{O}_{6} \mathrm{Si}_{2}[\mathrm{M}+\mathrm{Na}]^{+}$633.3983, found 633.3989 .

$(1 R, 2 R)-1-E t h y l-2-m e t h y l b u t-3-e n y l ~(2 R, 3 S, 4 R, 5 S, 6 S, 8 R)-3,9-d i(t e r t-$ butyldimethylsilanyloxy)-5-(4-methoxybenzyloxy)-2,4,6,8-tetramethylnonanoate (27)

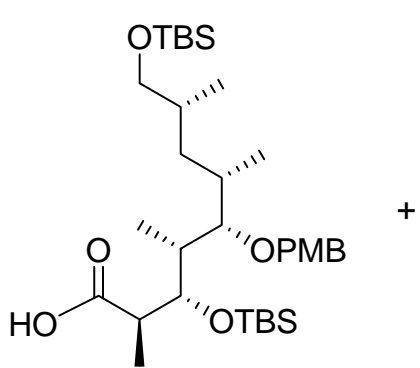

26<smiles>C=C[C@H](C)[C@H](O)CC</smiles>

9

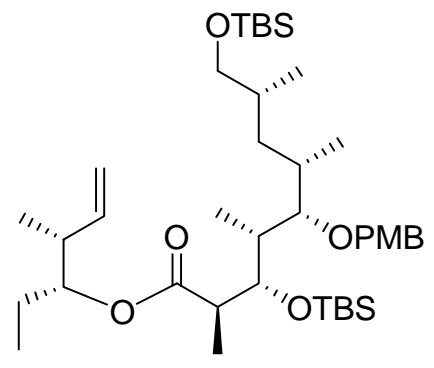

27

To a solution of carboxylic acid 26 (343 mg, $0.561 \mathrm{mmol})$ in THF (10 mL) at room 
temperature were added triethylamine $(117 \mu \mathrm{L}, 0.841 \mathrm{mmol})$ and 2,4,6-trichlorobenzoyl chloride $(109 \mu \mathrm{L}, 0.701 \mathrm{mmol})$. The reaction mixture was stirred for $3 \mathrm{~h}$ at room temperature. The solids were filtered off and washed with hexane $(3 \times 5 \mathrm{~mL})$. The solvent was evaporated under reduced pressure, and the residue was dissolved in benzene $(10 \mathrm{~mL})$. To this solution was added a solution of alcohol 11 (128 $\mathrm{mg}, 1.12$ mmol) and DMAP (96.0 $\mathrm{mg}, 0.785 \mathrm{mmol})$ in benzene $(2 \mathrm{~mL})$. After stirring $13 \mathrm{~h}$, the reaction mixture was diluted with ether $(20 \mathrm{~mL})$, and washed with aqueous saturated $\mathrm{NaHCO}_{3}(10 \mathrm{~mL})$ and aqueous saturated $\mathrm{NaCl}(10 \mathrm{~mL})$, and dried $\left(\mathrm{MgSO}_{4}\right)$ and concentrated. Purification of the residue by silica gel chromatography (hexane:EtOAc $=$ 9:1) afford the desired ester $27(376 \mathrm{mg}, 95 \%)$ as a colorless oil: $[\alpha]_{\mathrm{D}}{ }^{25.9}+7.90^{\circ}(c 1.84$, $\mathrm{CHCl}_{3}$ ); IR (film) $v_{\max } 2954,1728,1616,1512,1462,1377,1250,1173,1088,922,837$, 775, $675 \mathrm{~cm}^{-1} ;{ }^{1} \mathrm{H}$ NMR $\left(300 \mathrm{MHz}, \mathrm{CDCl}_{3}\right) \delta 0.07(\mathrm{~s}, 6 \mathrm{H}), 0.11(\mathrm{~m}, 6 \mathrm{H}), 0.88(\mathrm{~m}, 3 \mathrm{H})$, $0.94(\mathrm{~m}, 21 \mathrm{H}), 1.01(\mathrm{~m}, 9 \mathrm{H}), 1.19(\mathrm{~d}, J=7.1 \mathrm{~Hz}, 3 \mathrm{H}), 1.43(\mathrm{~m}, 2 \mathrm{H}), 1.59(\mathrm{~m}, 2 \mathrm{H}), 1.74$ (m, 1H), $1.85(\mathrm{~m}, 1 \mathrm{H}), 1.94(\mathrm{~m}, 1 \mathrm{H}), 2.43(\mathrm{ddd}, J=6.6,6.6,6.6 \mathrm{~Hz}, 1 \mathrm{H}), 2.81$ (dddd, $J$ $=6.9,6.9,6.9,6.9, \mathrm{~Hz}, 1 \mathrm{H}) 3.29(\mathrm{~m}, 2 \mathrm{H}), 3.63(\mathrm{dd}, J=9.8,4.4 \mathrm{~Hz}, 1 \mathrm{H}), 3.81(\mathrm{~s}, 3 \mathrm{H})$, $4.07(\mathrm{~m}, 1 \mathrm{H}), 4.50(\mathrm{~s}, 2 \mathrm{H}), 4.75(\mathrm{~m}, 1 \mathrm{H}), 5.03(\mathrm{~m}, 2 \mathrm{H}), 5.74(\mathrm{ddd}, J=17.4,10.3,7.4 \mathrm{~Hz}$, $1 \mathrm{H}), 6.88(\mathrm{~d}, J=8.6 \mathrm{~Hz}, 2 \mathrm{H}), 7.30(\mathrm{~d}, J=8.5 \mathrm{~Hz}, 2 \mathrm{H}) ;{ }^{13} \mathrm{C} \mathrm{NMR}\left(75 \mathrm{MHz}, \mathrm{CDCl}_{3}\right) \delta$ $175.4,158.8,139.8,131.4,128.8,115.1,113.6,84.0,77.8,77.2,74.0,73.7,67.8,55.1$, 
$43.4,40.5,40.5,35.7,33.2,26.2,26.0,24.0,19.0,18.5,18.3,17.3,15.2,14.1,10.8,9.7$, -3.8, -5.4; HRMS calcd for $\mathrm{C}_{40} \mathrm{H}_{74} \mathrm{O}_{6} \mathrm{Si}_{2}[\mathrm{M}+\mathrm{Na}]^{+}$729.4922, found 729.4916.

$(1 R, 2 R)-1-E t h y l-2-m e t h y l b u t-3-e n y l ~(2 R, 3 S, 4 R, 5 S, 6 S, 8 R)-3-(t e r t-$

Butyldimethylsilanyloxy)-9-hydroxy-5-(4-methoxybenzyloxy)-2,4,6,8-

tetramethylnonanoate (28)

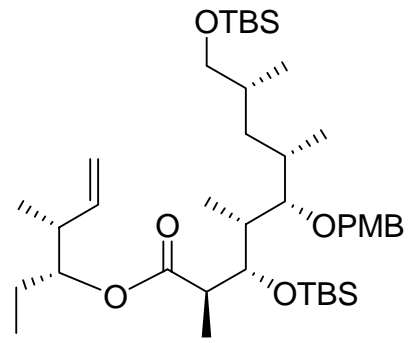

27

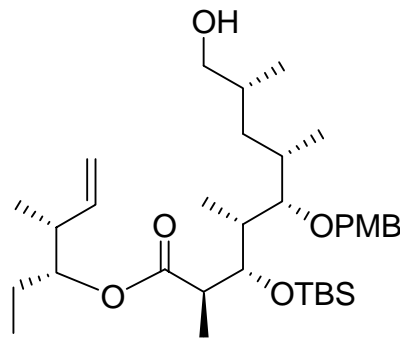

28

The ester 27 (376 $\mathrm{mg}, 0.532 \mathrm{mmol}$ ) obtained as described above was dissolved in $\mathrm{MeOH}(10 \mathrm{~mL})$. To this solution was added $D L$-10-camphorsulfonic acid (24.6 $\mathrm{mg}$, $0.106 \mathrm{mmol})$. The resulting solution was stirred at $0{ }^{\circ} \mathrm{C}$ for $1 \mathrm{~h}$. The reaction was terminated by addition of $\mathrm{Et}_{3} \mathrm{~N}(74 \mu \mathrm{L}, 0.53 \mathrm{mmol})$. After the solution was concentrated, purification by flash chromatography (hexane:EtOAc $=7: 1$ ) gave the desired primary alcohol $28(238 \mathrm{mg}, 76 \%)$ as a colorless liquid: $[\alpha]_{\mathrm{D}}^{26.5}+16.3^{\circ}\left(c 1.55, \mathrm{CHCl}_{3}\right)$; IR (film) $v_{\max } 3541,2958,1716,1612,1512,1462,1377,1250,1180,1049,926,833,775$ $\mathrm{cm}^{-1} ;{ }^{1} \mathrm{H}$ NMR $\left(300 \mathrm{MHz}, \mathrm{CDCl}_{3}\right) \delta 0.02(\mathrm{~d}, J=5.7 \mathrm{~Hz}, 6 \mathrm{H}), 0.75(\mathrm{t}, J=7.4 \mathrm{~Hz}, 3 \mathrm{H})$, 
$0.84(\mathrm{~s}, 9 \mathrm{H}), 0.87$ (d, $J=2.5 \mathrm{~Hz}, 3 \mathrm{H}), 0.91(\mathrm{~d}, J=2.9 \mathrm{~Hz}, 3 \mathrm{H}), 0.93(\mathrm{~m}, 6 \mathrm{H}), 1.11(\mathrm{~d}, J$ $=7.0 \mathrm{~Hz}, 3 \mathrm{H}), 1.31(\mathrm{~m}, 2 \mathrm{H}), 1.47(\mathrm{~m}, 2 \mathrm{H}), 1.57(\mathrm{~m}, 1 \mathrm{H}), 1.69(\mathrm{~m}, 1 \mathrm{H}), 1.81(\mathrm{~m}, 1 \mathrm{H})$, $2.29(\mathrm{dddd}, J=13.2,6.5,6.5,6.5 \mathrm{~Hz}, 1 \mathrm{H}), 2.63(\mathrm{dddd}, J=7.0,7.0,7.0,7.0, \mathrm{~Hz}, 1 \mathrm{H})$, 2.81(s, 1H), $3.13(\mathrm{dd}, J=8.7,1.9 \mathrm{~Hz}, 1 \mathrm{H}), 3.34(\mathrm{~m}, 1 \mathrm{H}), 3.56(\mathrm{dd}, J=11.1,3.2 \mathrm{~Hz}, 1 \mathrm{H})$, 3.69 (s, 3H), $3.83(\mathrm{~d}, J=8.5 \mathrm{~Hz}, 1 \mathrm{H}), 4.40$ (ddd, $J=10.6,10.6,10.6 \mathrm{~Hz}, 2 \mathrm{H}), 4.65$ (m, 1H), $4.93(\mathrm{~m}, 2 \mathrm{H}), 5.62(\mathrm{ddd}, J=17.5,10.3,7.4 \mathrm{~Hz}, 1 \mathrm{H}), 6.77(\mathrm{~d}, J=8.6 \mathrm{~Hz}, 2 \mathrm{H}), 7.17$ $(\mathrm{d}, J=8.4 \mathrm{~Hz}, 2 \mathrm{H}) ;{ }^{13} \mathrm{C} \mathrm{NMR}\left(75 \mathrm{MHz}, \mathrm{CDCl}_{3}\right) \delta 176.5,158.9,139.6,131.3,128.9$, $115.4,113.6,85.3,78.4,75.1,73.6,65.9,55.2,44.5,40.7,40.6,32.8,32.5,32.4,26.2$, 24.2, 18.8, 18.5, 18.1, 16.0, 14.9, 9.9, 9.7, -3.4, -3.5; HRMS calcd for $\mathrm{C}_{34} \mathrm{H}_{60} \mathrm{O}_{6} \mathrm{Si}[\mathrm{M}+$ $\mathrm{Na}]^{+}$615.4057, found 615.4053 .

(1R,2R)-1-Ethyl-2-methyl-but-3-enyl (2R,3S,4R,5S,6S,8R)-3-(tert-

butyldimethylsilanyloxy)-5-(4-methoxybenzyloxy)-2,4,6,8-tetramethyl-9-oxoundec-

10-enoate (29)

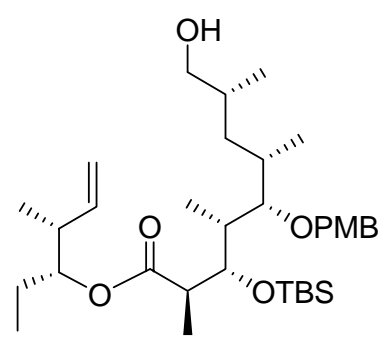

28
1) $\mathrm{DMP}, \mathrm{CH}_{2} \mathrm{Cl}_{2}$

2) $\mathrm{CH}_{2}=\mathrm{CHMgBr}$

3) $\mathrm{DMP}, \mathrm{CH}_{2} \mathrm{Cl}_{2}$

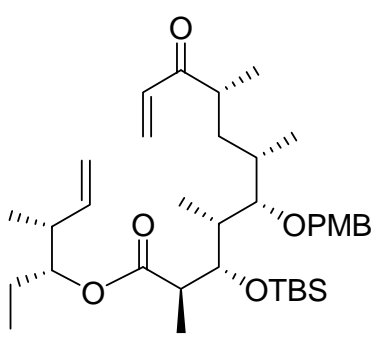

29 
The alcohol 28 (196 mg, $0.331 \mathrm{mmol}$ ) obtained as described in the previous procedure was dissolved in $\mathrm{CH}_{2} \mathrm{Cl}_{2}(10 \mathrm{~mL})$. To this solution was added Dess-Martin periodinane (DMP) $(281 \mathrm{mg}, 0.662 \mathrm{mmol})$. The resulting solution was stirred for $30 \mathrm{~min}$ at room temperature. After the reaction was completed, aqueous saturated $\mathrm{NaHCO}_{3}(10 \mathrm{~mL})$ was added. The mixture was extracted with $\mathrm{CH}_{2} \mathrm{Cl}_{2}(3 \times 20 \mathrm{~mL})$. The organic layer was separated, dried $\left(\mathrm{MgSO}_{4}\right)$, and concentrated. Purification by flash chromatography (hexane:EtOAc $=4: 1)$ offered the desired aldehyde $29(151 \mathrm{mg}, 77 \%)$ as a yellow liquid: ${ }^{1} \mathrm{H} \mathrm{NMR}\left(300 \mathrm{MHz}, \mathrm{CDCl}_{3}\right) \delta 0.09(\mathrm{~s}, 6 \mathrm{H}), 0.86(\mathrm{t}, J=7.2 \mathrm{~Hz}, 3 \mathrm{H}), 0.93(\mathrm{~s}, 9 \mathrm{H})$, $1.01(\mathrm{~m}, 9 \mathrm{H}), 1.10(\mathrm{~d}, J=6.9 \mathrm{~Hz}, 3 \mathrm{H}), 1.19(\mathrm{~d}, J=7.1 \mathrm{~Hz}, 3 \mathrm{H}), 1.56(\mathrm{~m}, 3 \mathrm{H}), 1.83(\mathrm{~m}$, 3H), $2.42(\mathrm{~m}, 2 \mathrm{H}), 2.74(\mathrm{dddd}, J=7.0,7.0,7.0,7.0 \mathrm{~Hz}, 1 \mathrm{H}) 3.24(\mathrm{dd}, J=7.3,2.9 \mathrm{~Hz}$, 1H), 3.80 (s, 3H), 3.95 (dd, $J=7.3,2.7 \mathrm{~Hz}, 1 \mathrm{H}), 4.49$ (ddd, $J=10.6,10.6,10.6 \mathrm{~Hz}, 2 \mathrm{H})$, $4.74(\mathrm{~m}, 1 \mathrm{H}), 5.03(\mathrm{~m}, 2 \mathrm{H}), 5.73(\mathrm{ddd}, J=17.5,10.3,7.4 \mathrm{~Hz}, 1 \mathrm{H}), 6.87$ (d, $J=8.5 \mathrm{~Hz}$, 2H), $7.27(\mathrm{~d}, J=8.5 \mathrm{~Hz}, 2 \mathrm{H}), 9.58(\mathrm{~d}, J=3.4 \mathrm{~Hz}, 1 \mathrm{H}) ;{ }^{13} \mathrm{C} \mathrm{NMR}\left(75 \mathrm{MHz}, \mathrm{CDCl}_{3}\right) \delta$ $206.2,175.5,159.0,139.8,131.1,128.9,115.2,113.6,84.6,77.9,74.7,73.7,55.2,44.1$, $44.1,40.8,40.6,33.1,32.0,26.2,24.1,18.5,17.5,15.2,15.1,18.5,17.5,15.2,15.0$, $10.3,9.7,-3.6,-3.7$.

To a stirred solution of the aldehdyde $(151 \mathrm{mg}, 0.255 \mathrm{mmol})$ prepared as described in 
the previous procedure in THF $(10 \mathrm{~mL})$ was added $1 \mathrm{M}$ vinylmagnesium bromide $(1.0$ $\mathrm{mL}$, in THF, $1.0 \mathrm{mmol}$ ) at room temperature. After $20 \mathrm{~min}$, the reaction mixture was diluted by adding $\mathrm{Et}_{2} \mathrm{O}(10 \mathrm{~mL})$, and then added a saturated aqueous $\mathrm{NH}_{4} \mathrm{Cl}$ solution $(10$ $\mathrm{mL}$ ). The organic solution was separated, and the aqueous layer was extracted with ether $(3 \times 15 \mathrm{~mL})$. The organic solutions were combined, dried $\left(\mathrm{MgSO}_{4}\right)$, and concentrated. Purification of the residue by flash chromatography (hexane:EtOAc $=4: 1$ ) afforded the desired alcohol (132 $\mathrm{mg}, 82 \%)$ as a colorless oil.

The alcohol (132 mg, $0.213 \mathrm{mmol})$ obtained as described in the previous procedure was dissolved in $\mathrm{CH}_{2} \mathrm{Cl}_{2}(10 \mathrm{~mL})$. To this solution was added Dess-Martin periodinane (DMP) (180 $\mathrm{mg}, 0.426 \mathrm{mmol})$, and the resulting solution was stirred for $30 \mathrm{~min}$ at room temperature. After the reaction was completed, aqueous saturated $\mathrm{NaHCO}_{3}(10 \mathrm{~mL})$ was added and the mixture was extracted with $\mathrm{CH}_{2} \mathrm{Cl}_{2}(3 \times 15 \mathrm{~mL})$. The organic layer was separated, dried $\left(\mathrm{MgSO}_{4}\right)$, and concentrated. Purification by flash chromatography (hexane:EtOAc $=5: 1)$ offered the desired ketone $29(128 \mathrm{mg}, 99 \%)$ as a yellow liquid: $[\alpha]_{\mathrm{D}}^{25.7}+18.1^{\circ}\left(c 1.20, \mathrm{CHCl}_{3}\right)$; IR (film) $v_{\max } 2926,1724,1612,1512,1462,1377$, 1250, 1176, 1049, 833, 775, $679 \mathrm{~cm}^{-1} ;{ }^{1} \mathrm{H}$ NMR $\left(300 \mathrm{MHz}, \mathrm{CDCl}_{3}\right) \delta 0.05(\mathrm{~d}, J=2.7$ $\mathrm{Hz}, 6 \mathrm{H}), 0.87$ (t, $J=7.4 \mathrm{~Hz}, 3 \mathrm{H}), 0.91(\mathrm{~s}, 9 \mathrm{H}), 0.99(\mathrm{~m}, 9 \mathrm{H}), 1.12(\mathrm{~d}, J=7.0 \mathrm{~Hz}, 3 \mathrm{H})$, 
$1.19(\mathrm{~d}, J=7.1 \mathrm{~Hz}, 3 \mathrm{H}), 1.76 \sim 1.43(\mathrm{~m}, 4 \mathrm{H}), 1.85(\mathrm{~m}, 1 \mathrm{H}), 1.94(\mathrm{~m}, 1 \mathrm{H}), 2.42$ (dddd, $J$ $=13.1,6.4,6.4,6.4 \mathrm{~Hz}, 1 \mathrm{H}), 2.74(\mathrm{dddd}, J=6.9,6.9,6.9,6.9 \mathrm{~Hz}, 1 \mathrm{H}), 2.88(\mathrm{~m}, 1 \mathrm{H})$, $3.22(\mathrm{dd}, J=6.4,3.7 \mathrm{~Hz}, 1 \mathrm{H}), 3.80(\mathrm{~s}, 3 \mathrm{H}), 3.99(\mathrm{dd}, J=6.5,3.5 \mathrm{~Hz}, 1 \mathrm{H}), 4.47$ (ddd, $J$ $=10.9,10.9,10.9 \mathrm{~Hz}, 2 \mathrm{H}), 4.75(\mathrm{~m}, 1 \mathrm{H}), 5.02(\mathrm{~m}, 2 \mathrm{H}), 5.74(\mathrm{~m}, 2 \mathrm{H}), 6.29(\mathrm{dd}, J=17.3$, $1.3 \mathrm{~Hz}, 1 \mathrm{H}), 6.60(\mathrm{dd}, J=17.3,10.4 \mathrm{~Hz}, 1 \mathrm{H}), 6.86(\mathrm{~d}, J=8.6 \mathrm{~Hz}, 2 \mathrm{H}), 7.27(\mathrm{~d}, J=8.5$ $\mathrm{Hz}, 2 \mathrm{H}) ;{ }^{13} \mathrm{C} \mathrm{NMR}\left(75 \mathrm{MHz}, \mathrm{CDCl}_{3}\right) \delta 204.0,175.4,178.9,139.9,134.3,131.2,128.9$, $128.0,115.6,84.4,77.9,74.3,73.8,55.2,44.0,42.1,40.6,40.5,33.9,33.3,26.2,24.1$, 18.5, 18.1, 17.1, 15.1, 14.7, 10.7, 9.7, -3.7, -3.8; HRMS calcd for $\mathrm{C}_{36} \mathrm{H}_{60} \mathrm{O}_{6} \mathrm{Si}[\mathrm{M}+\mathrm{Na}]^{+}$ 639.4057, found 639.4053. 


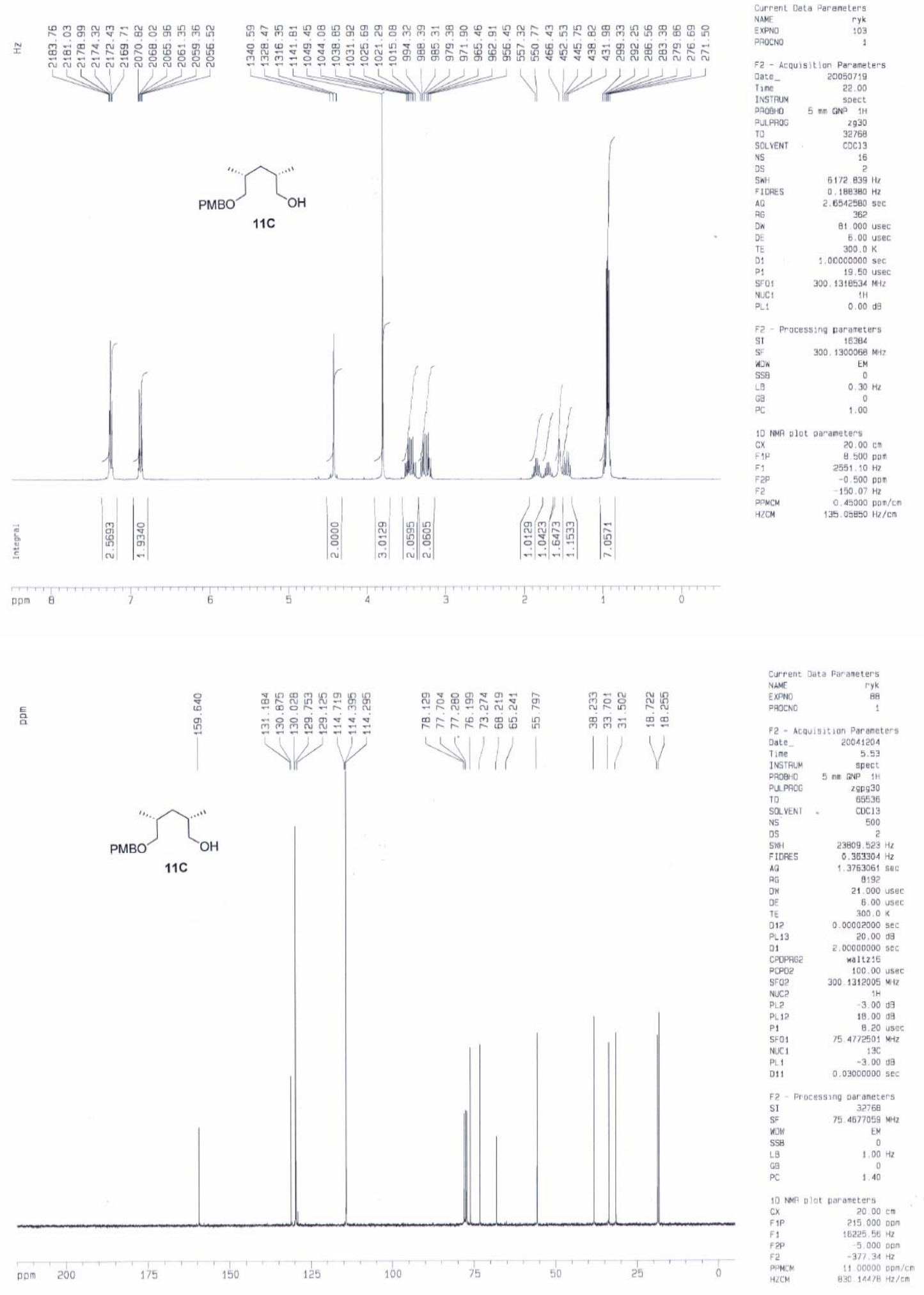




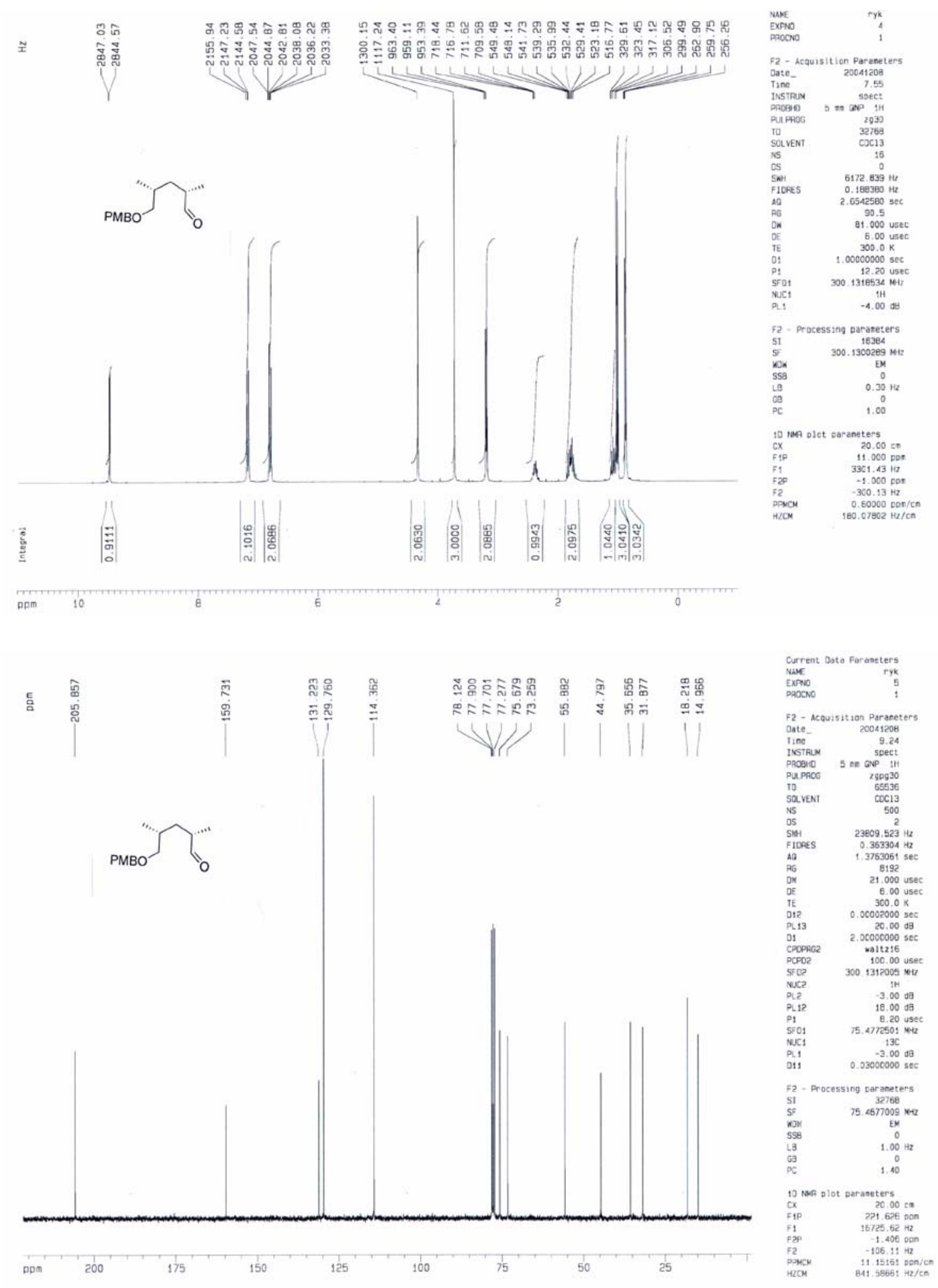




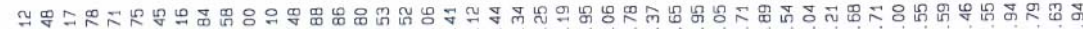

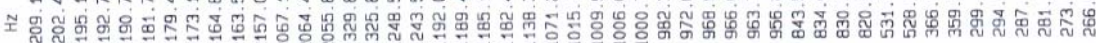

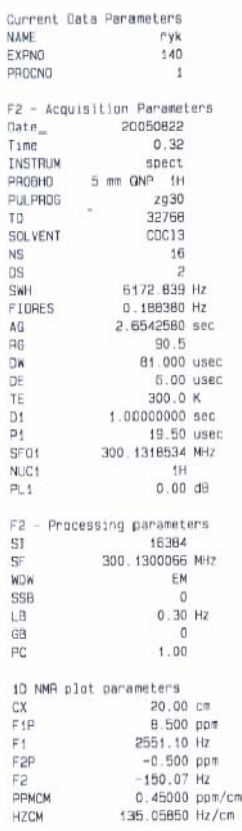

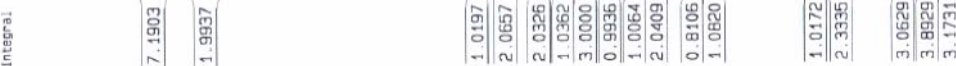

pom 8

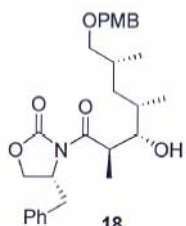

1,1

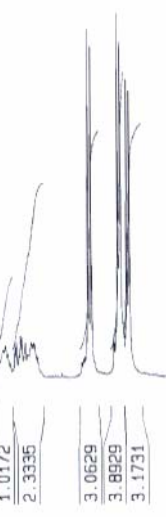

?
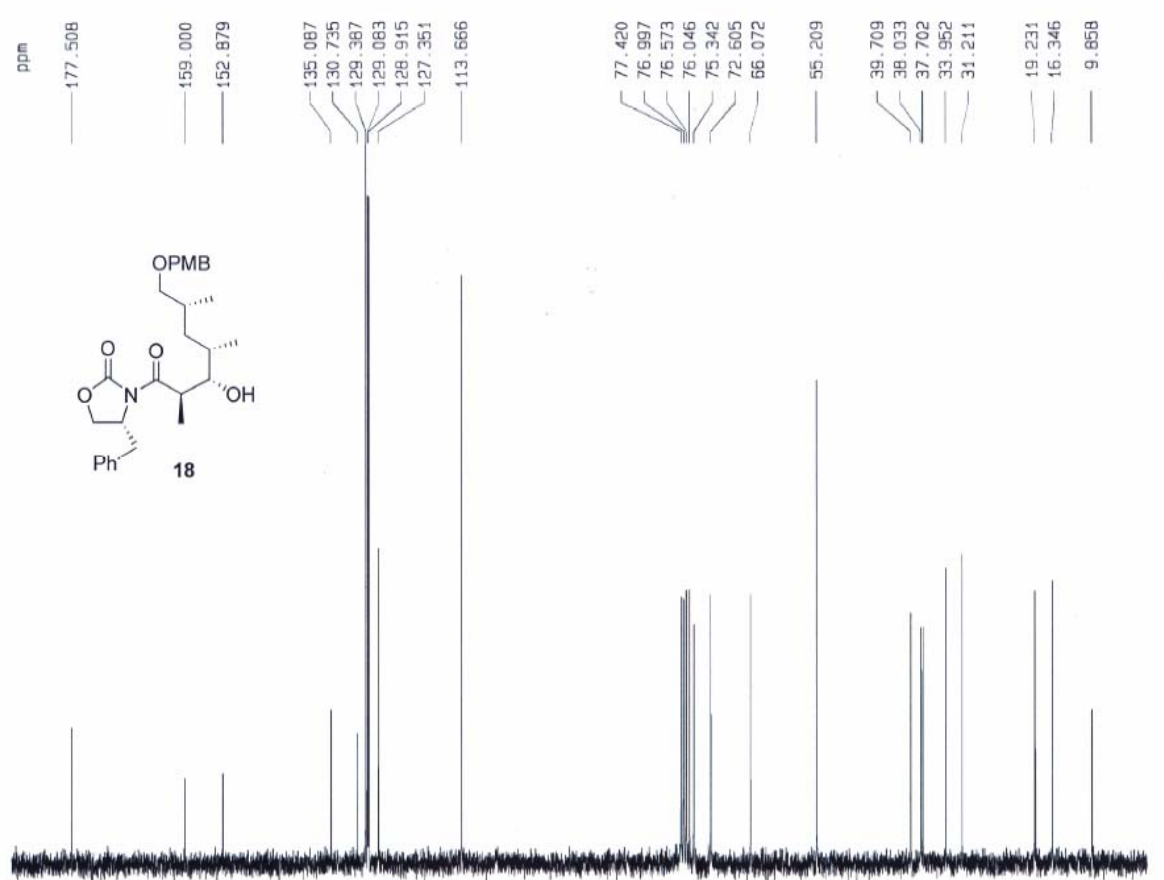

ppm

160

140

120

100

80

60

40

20

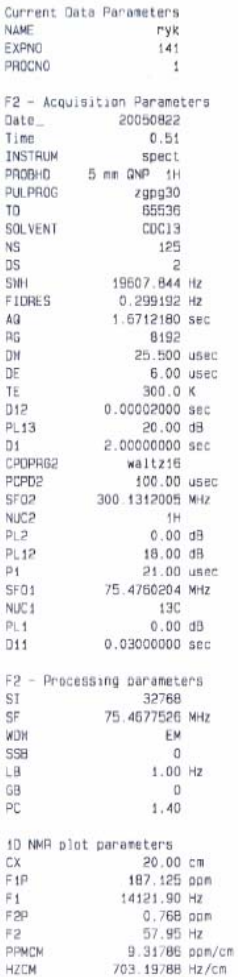




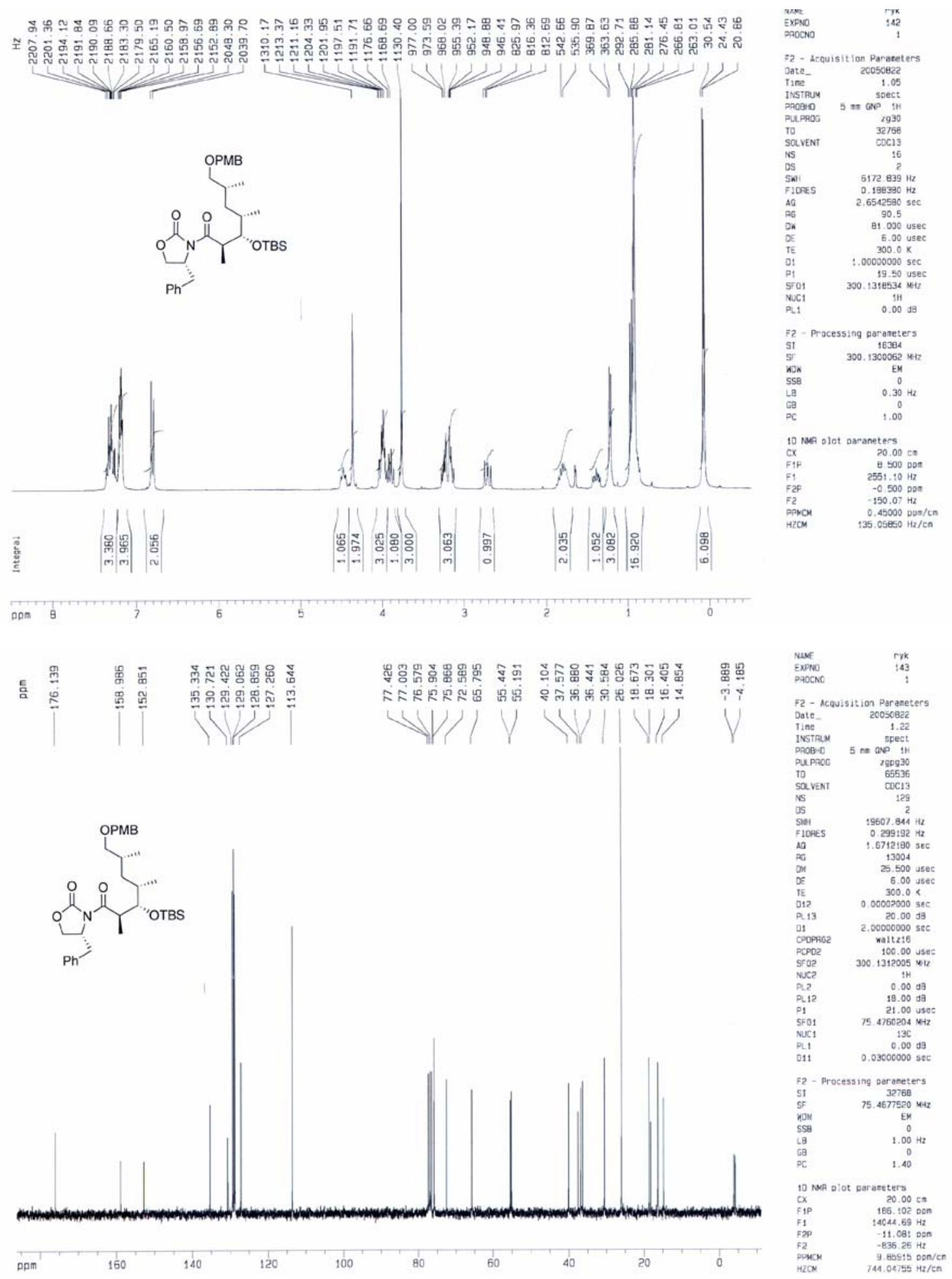




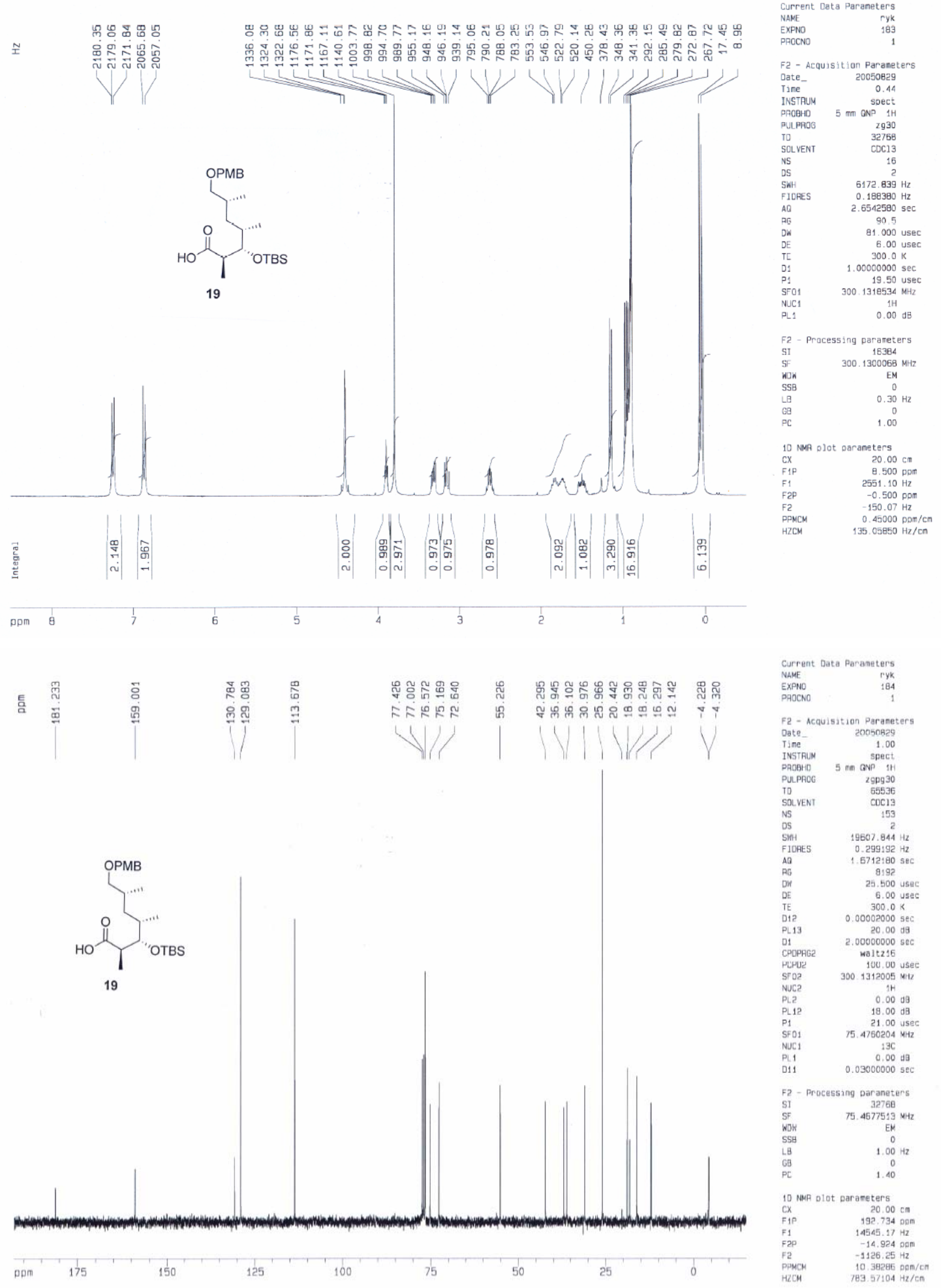



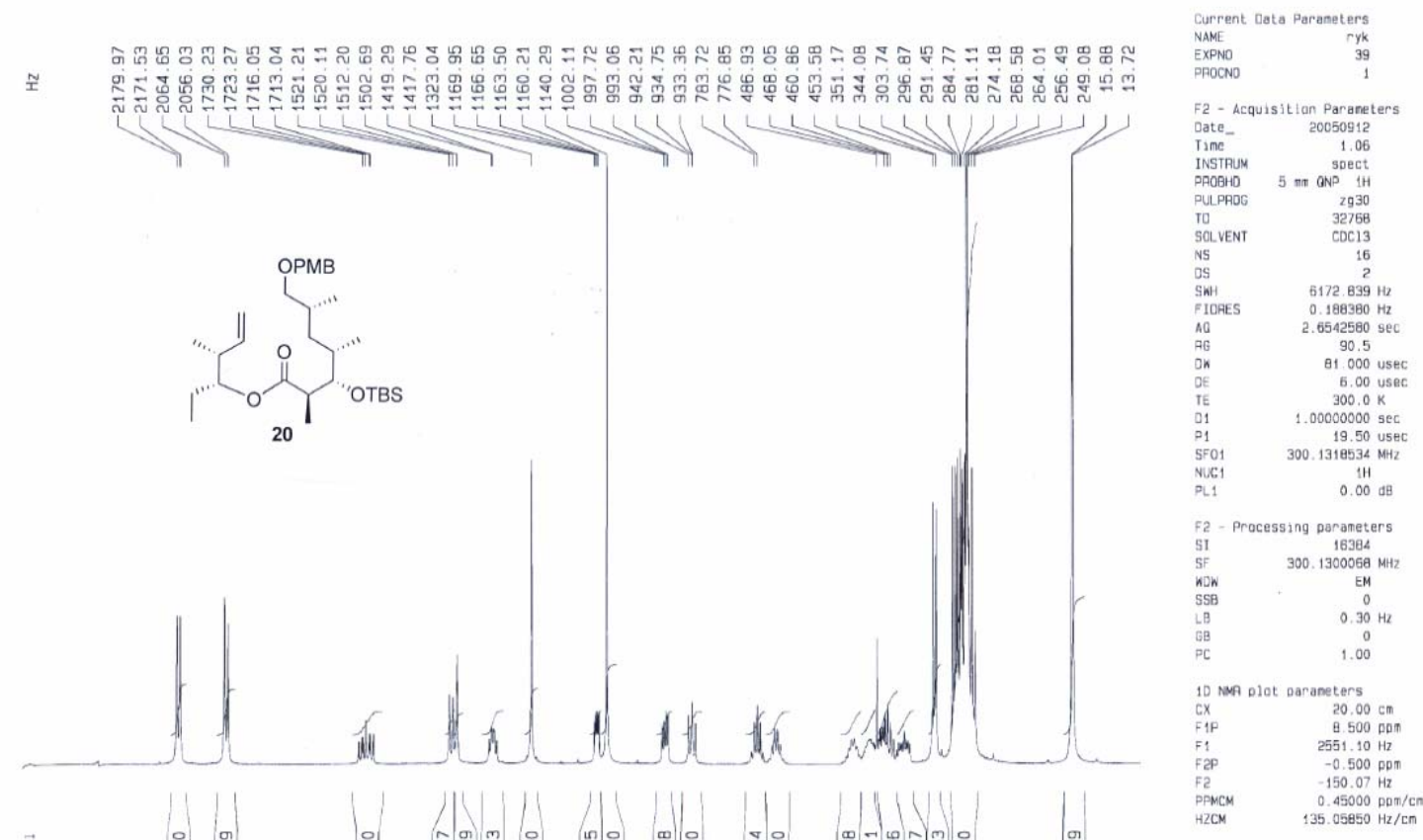

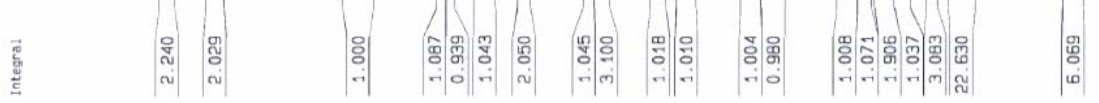
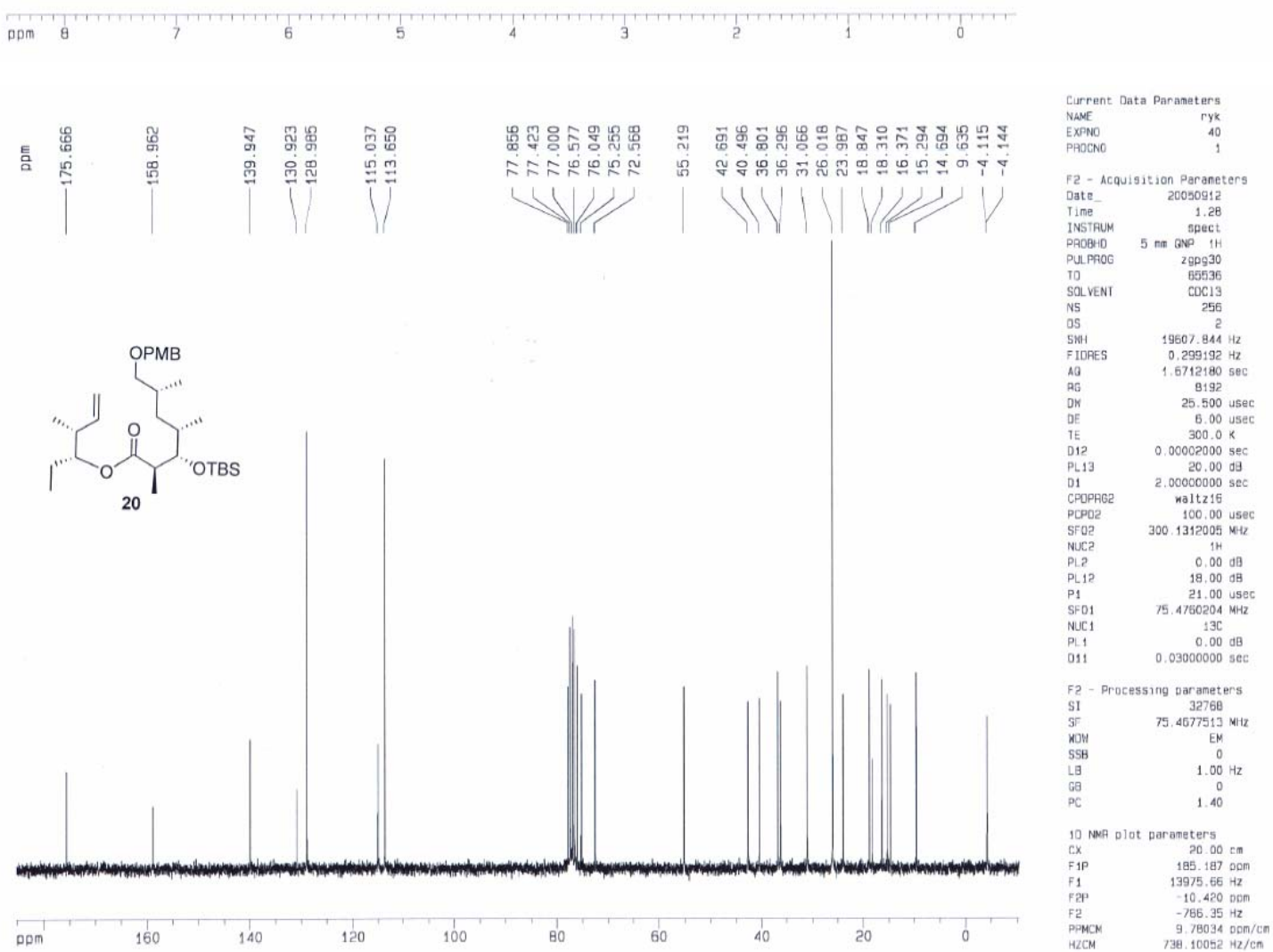

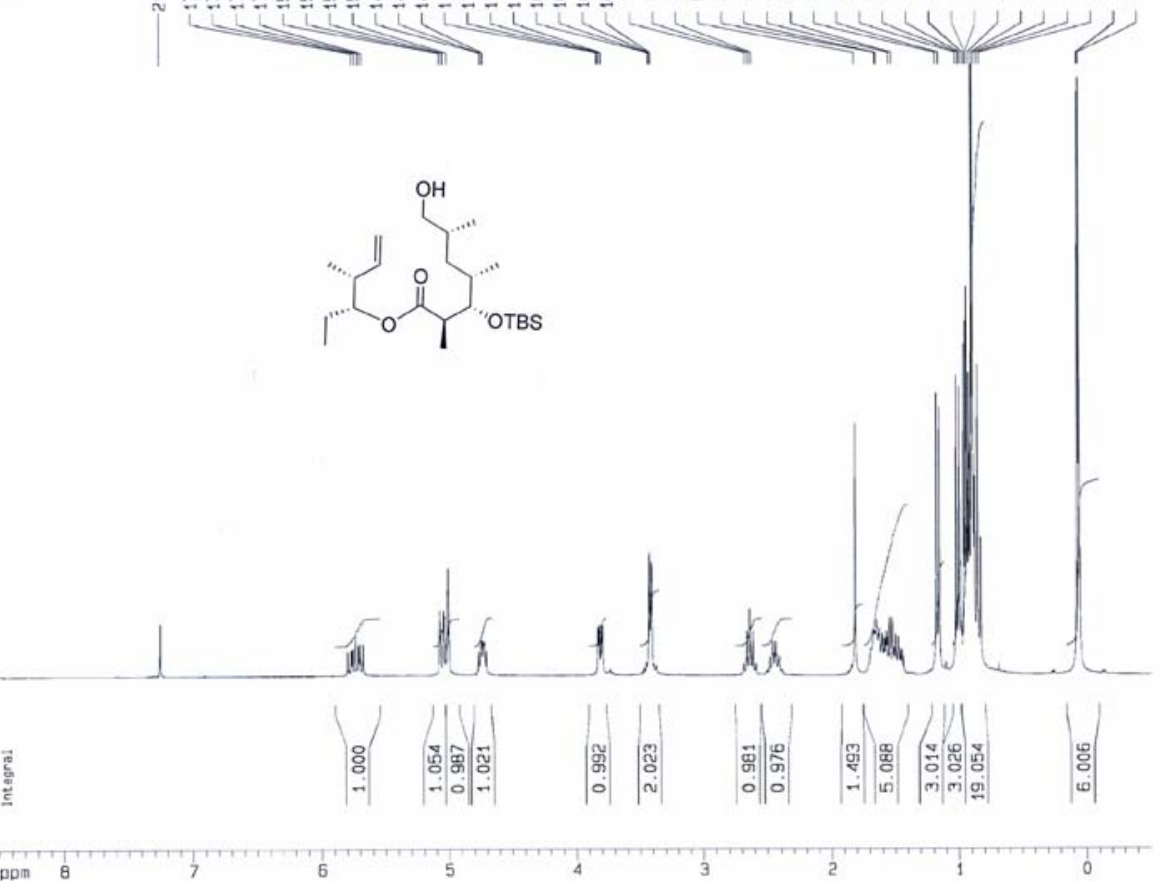

Current Dato Paraneters
NWE
EXPNo
PRCCNO

F2 - Acquisition Paraneters

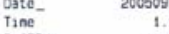

INStrus soect

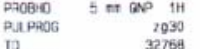

SoLVET

\begin{tabular}{lr} 
NS & COC13 \\
OS & 16 \\
\hline & 2
\end{tabular}

$\begin{array}{ll}S \text { SW } & 6172.839 \mathrm{~Hz} \\ \text { E1CFes } & 0.188980 \mathrm{~Hz}\end{array}$

$46 \quad 2.6542580 \mathrm{sec}$

91.000 usec
5.00 usec

$3.00000000 \mathrm{Kec}$
$\mathrm{se}$

For $\quad 300.1318534$ Net?

Nec1

F2 - Pracessing parazeters

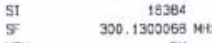

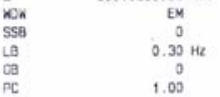

10 Nan olat oaraneters
CX $20.00 \mathrm{cn}$

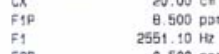

$\begin{array}{lll}F 2 \mathrm{P} & -0.500 \mathrm{pa} \\ 72 & -150,07\end{array}$

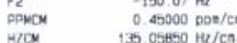
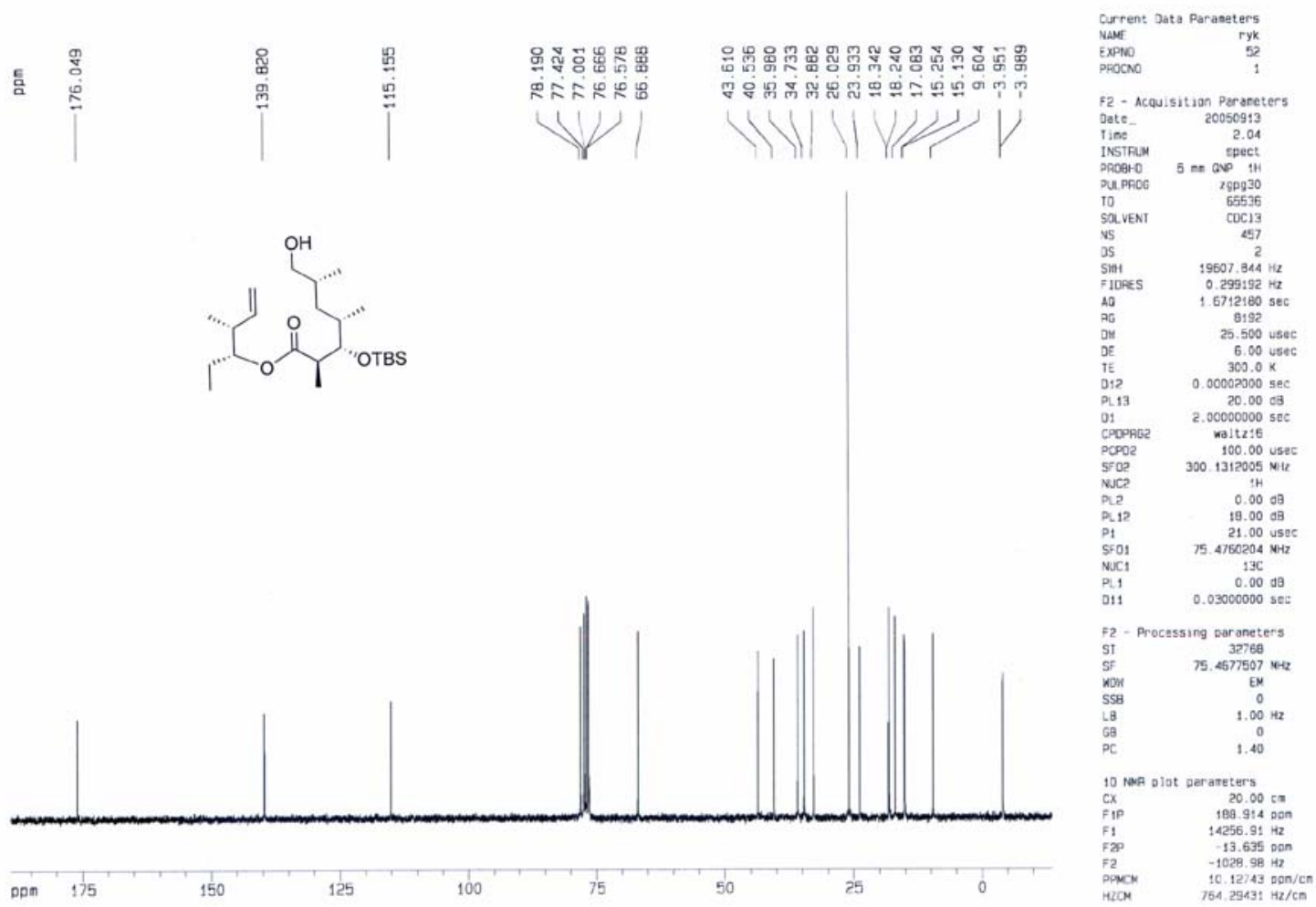


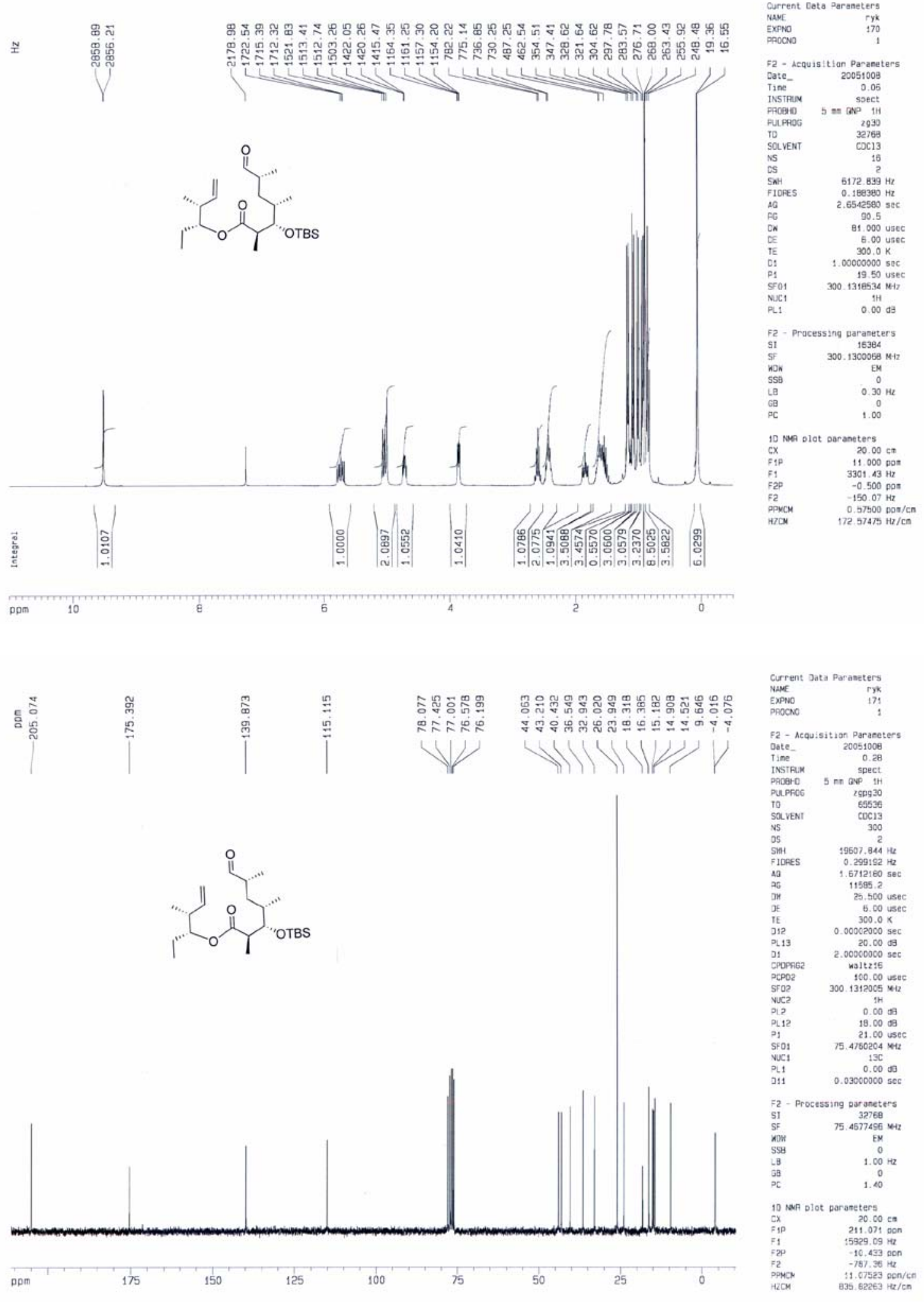




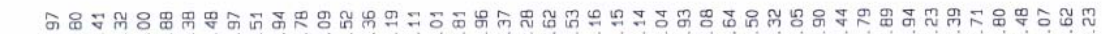

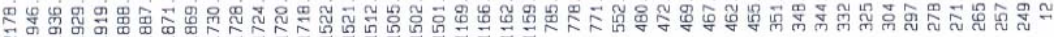
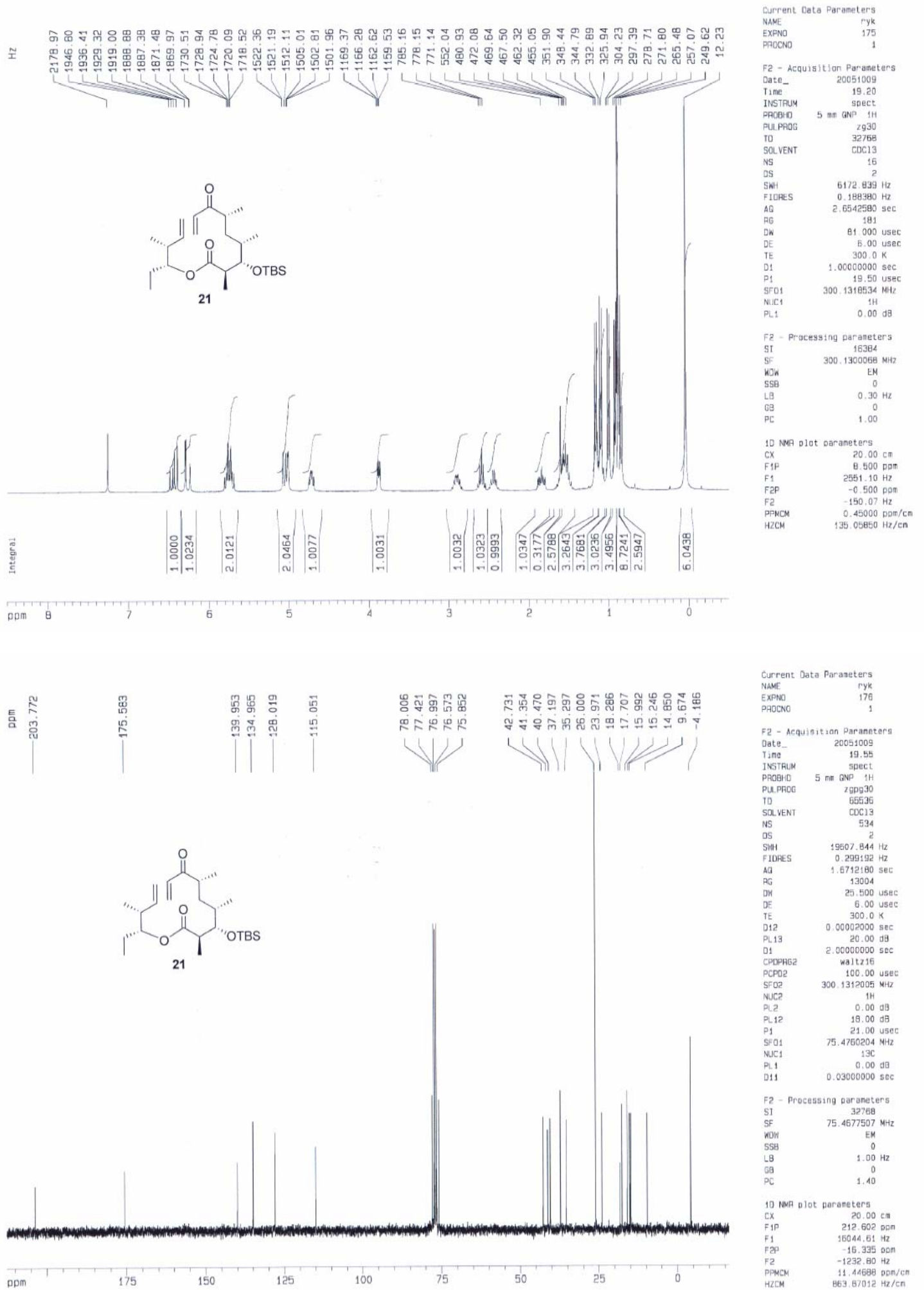


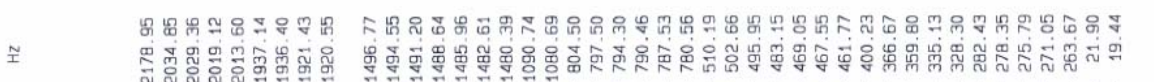

Current Data Parameters
NAME
Syk

EXPNO
PAOCNO

F2 - Acquisition Paraneters
Date_ $\quad 20051010$

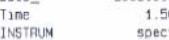

PROBHO $5 \mathrm{~mm}$ ove $1 \mathrm{H}$
PULPROG
2930

$\begin{array}{lr}\text { TD } & 39768 \\ \text { SOLVENT } & \text { CDC13 } \\ \text { NS } & 32\end{array}$
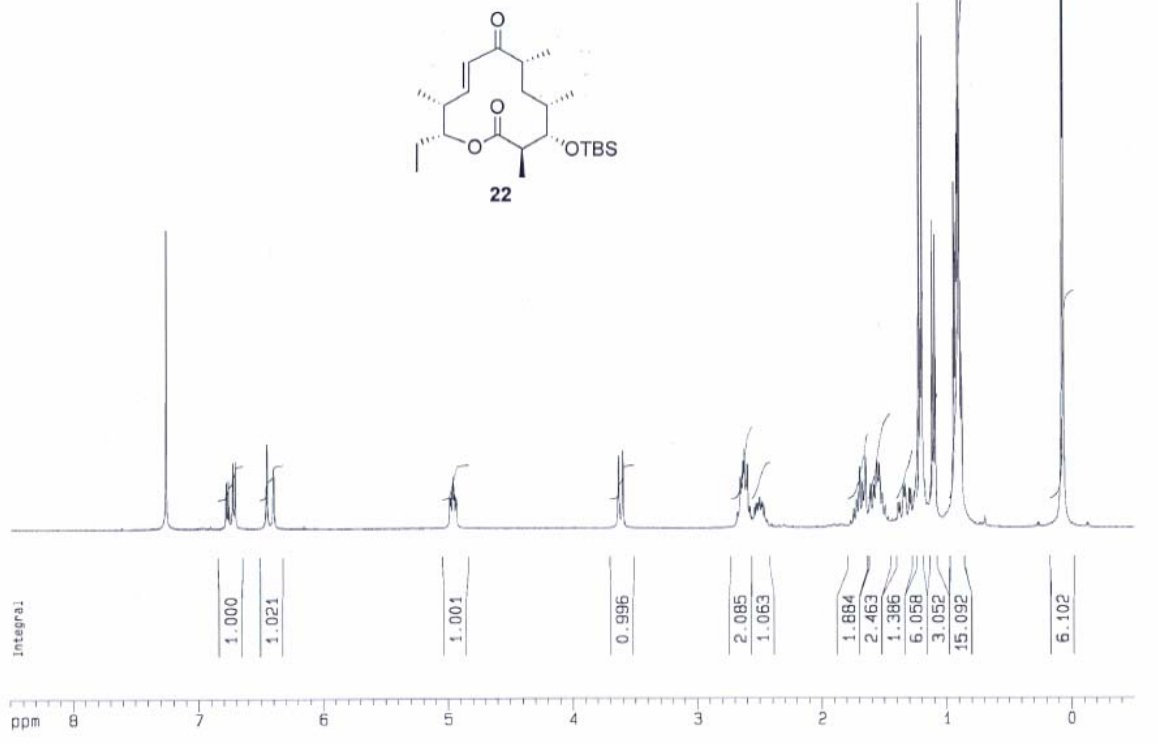

6172. $839 \mathrm{~Hz}$

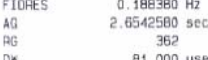

$\begin{array}{lr}D K & 91.000 \text { usec } \\ D E & 6.00 \text { usec } \\ D & 300.0 \%\end{array}$

$1,00000000 \mathrm{sec}$
$19.50 \mathrm{uset}$

SF01 $300.1318534 \mathrm{MHz}$

$\begin{array}{ll}\text { NuC1 } & 1 \mathrm{H} \\ \text { PL.1 } & 0.00 \mathrm{~dB}\end{array}$

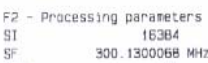

$\begin{array}{cc}\text { ST } & 300.1300068 \\ \text { KSO } & \text { EM } \\ \text { SSB } & 0 \\ 0.30\end{array}$

$\begin{array}{ll}\mathrm{CQ} & 0 \\ \mathrm{PC} & 1.00\end{array}$

10. NMA Dlot parameters

$\begin{array}{ll}F 1 P & 8.500 \mathrm{por} \\ \mathrm{F1} & 2551.10 \mathrm{~Hz}\end{array}$

$\begin{array}{ll}F 2 \mathrm{P} & -0.500 \mathrm{pDm} \\ \mathrm{F2} & -150.07 \mathrm{~Hz}\end{array}$

HZCM $\quad 135.05850 \mathrm{~Hz} / \mathrm{cm}$

镸

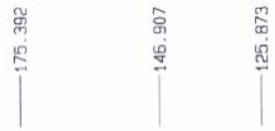

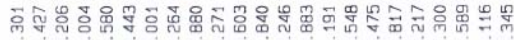

Current Data Parazeters
VaME
EXPNO

F2 - Acquisition Paraneters

200510.0
Tine

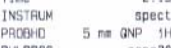

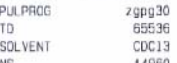

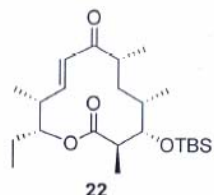

$19607.044 \mathrm{HIZ}$
$0.299192 \mathrm{~Hz}$

$\begin{array}{ll}19 & 1.6712180 \mathrm{se}\end{array}$

25.500 usec
OH
DE

$\begin{array}{ll}\text { IE } & 300.0 \mathrm{~K} \\ \mathrm{D} & 0.00002000 \mathrm{sec}\end{array}$

2.13 $20.00 \mathrm{dg}$

$\begin{array}{ll}\text { CPOPAG2 } & \text { Nalt t216 } \\ \text { PCPO? } & 100.00 \text { use }\end{array}$

SF02 300.1312005 MHE

$\begin{array}{lr}1 \mathrm{H} \\ \text { NUC2 } & 0.00 \mathrm{~dB} \\ \text { PL2 } & 18.00 \mathrm{~dB}\end{array}$

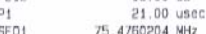

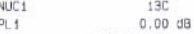

$0.03000000 \mathrm{sec}$

F2 - Processing paraneters
SI 32768
SF
FF

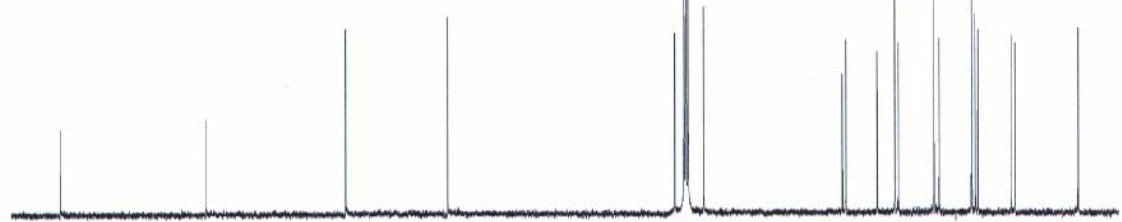

$\begin{array}{cc}\text { EM } \\ \text { SSB } & 0 \\ \mathrm{BS} & 1.00 \mathrm{~Hz}\end{array}$

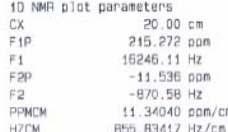




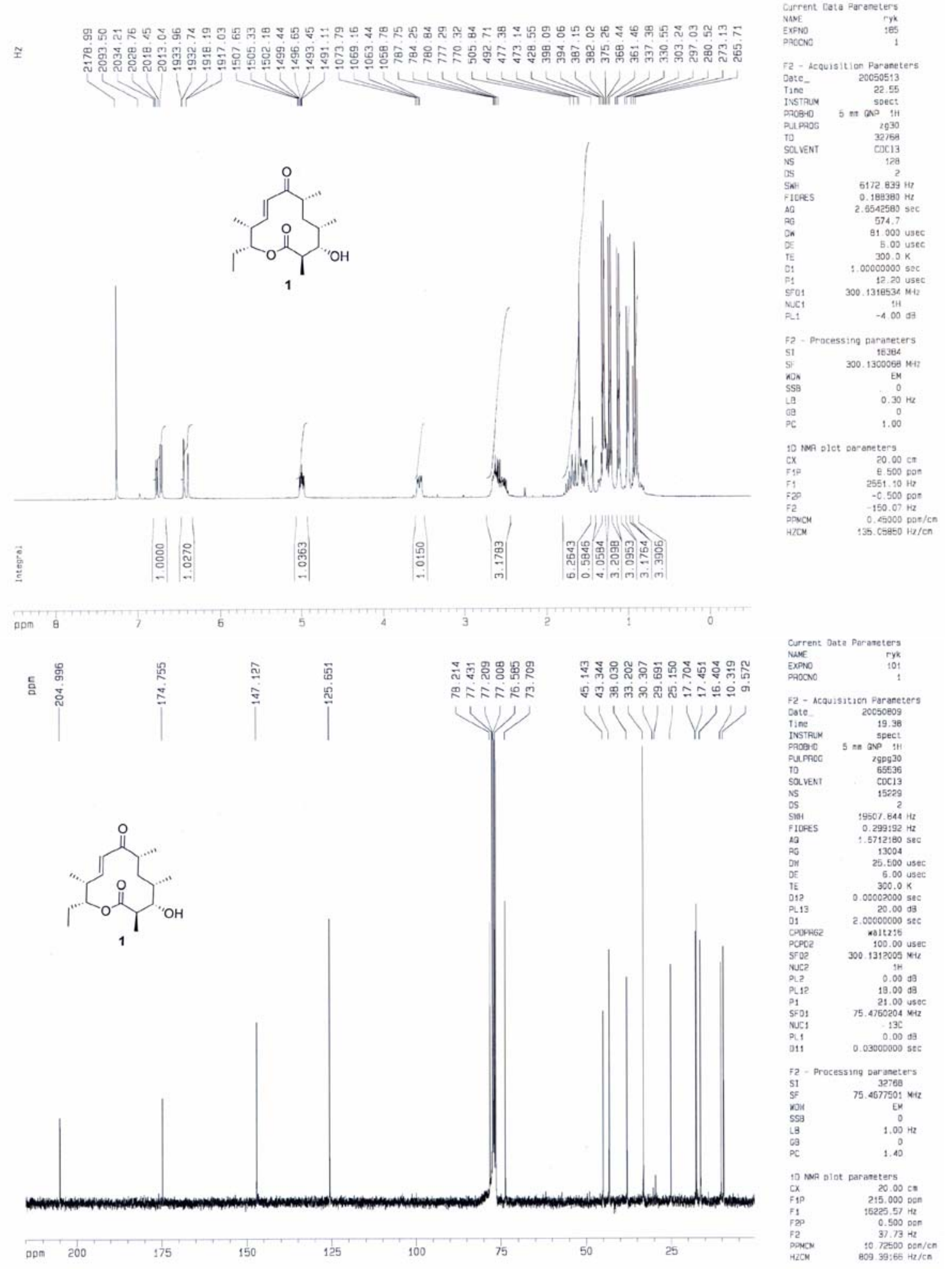



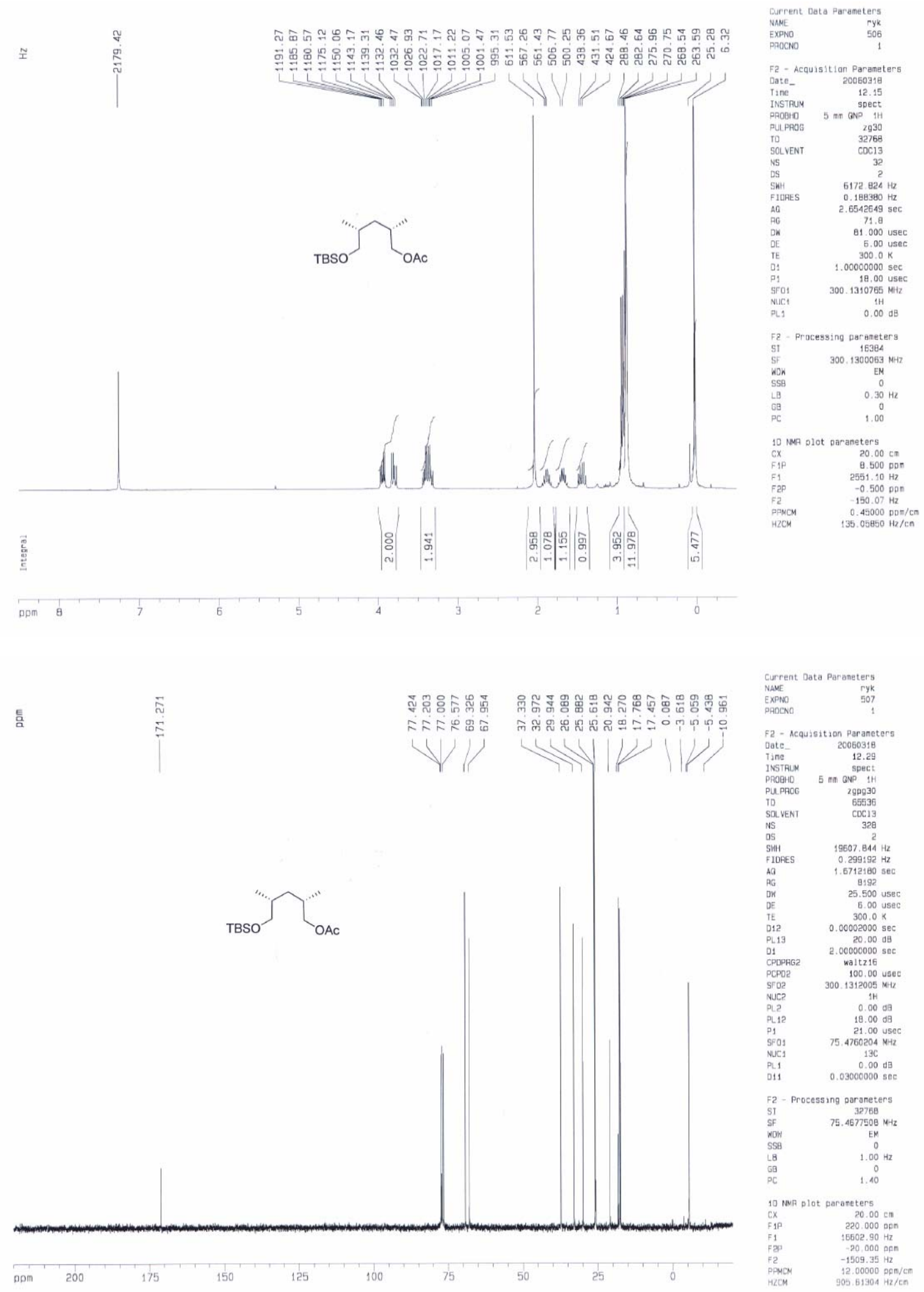


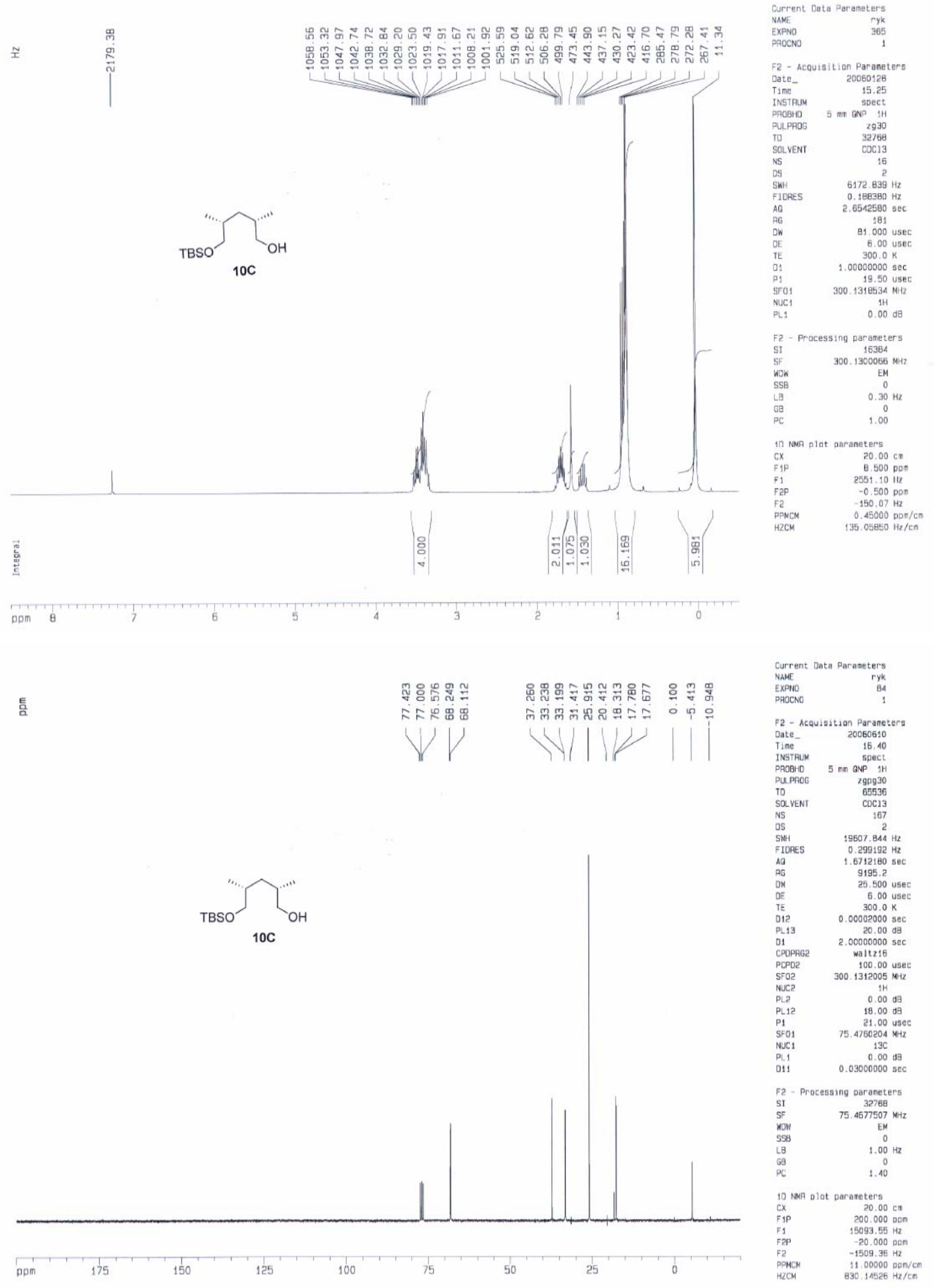



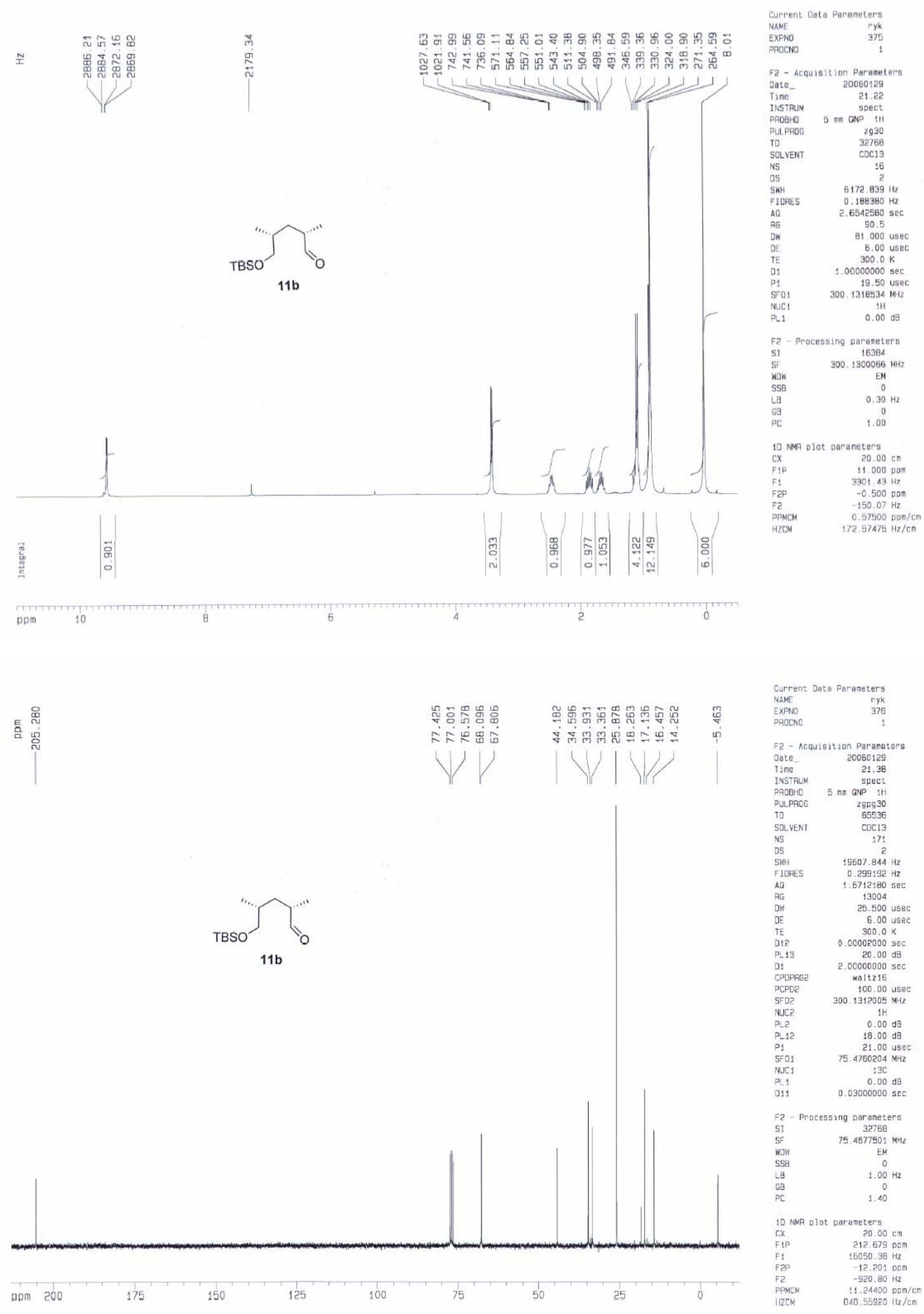


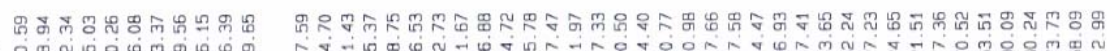

Current Data Paraneters
NAME

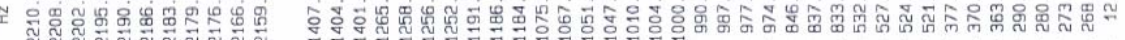

F2- Acquisition Paraneter
Date- $\quad 20060128$

$\begin{array}{lr}\text { Time } & 15.56 \\ \text { INSTRUY } & \text { spect }\end{array}$

2930
PULPRDG
TO

$\begin{array}{lr}\text { SOLVENT } & \text { CDC13 } \\ \text { NS } & 16 \\ \text { OS } & \text { S }\end{array}$<smiles>CC(C[C@H](C)C[SeH])[C@@H](C)[C@@H](C)C(=O)N1C(=O)OC[C@H]1Cc1ccccc1</smiles>
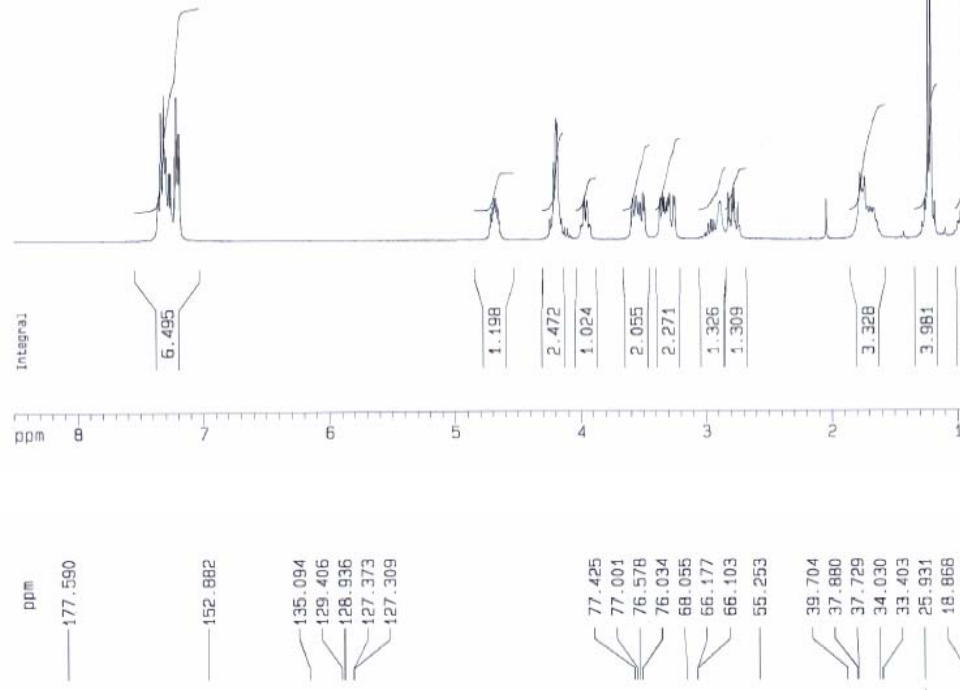

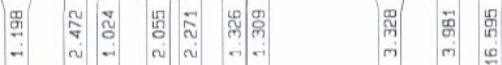

$6172.839 \mathrm{H} 2$
0.1889800

$\begin{array}{ll}\text { FIDRES } & 0.188380 \mathrm{~Hz} \\ A Q & 2.6542500 \mathrm{sec}\end{array}$

PG
DW
DE
DE

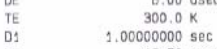

$\begin{array}{ll}\text { P1 } & 19.50 \text { usec } \\ \text { SF01 } & 300.1318534 \\ \text { MHIz }\end{array}$

$\begin{array}{lll}\text { NuC1 } & \text { IH } \\ \text { PL1 } & 0.00 \mathrm{~dB}\end{array}$

F2 - Processing paraneters

$\begin{array}{lrl}\text { SI } & 16384 \\ \text { SF } & 300.1300063 \mathrm{M}-12\end{array}$

SSB

然

10 NMA plat parameters
CX $20.00 \mathrm{~cm}$

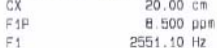

$\begin{array}{ll}\text { F2P } & -0.500 \mathrm{ppr} \\ \mathrm{FP} & -150.07 \mathrm{pr}\end{array}$

PPNCM $\quad 0.45000 \mathrm{pDr} / \mathrm{Cm}$

3. $05850 \mathrm{~Hz} / \mathrm{cm}$

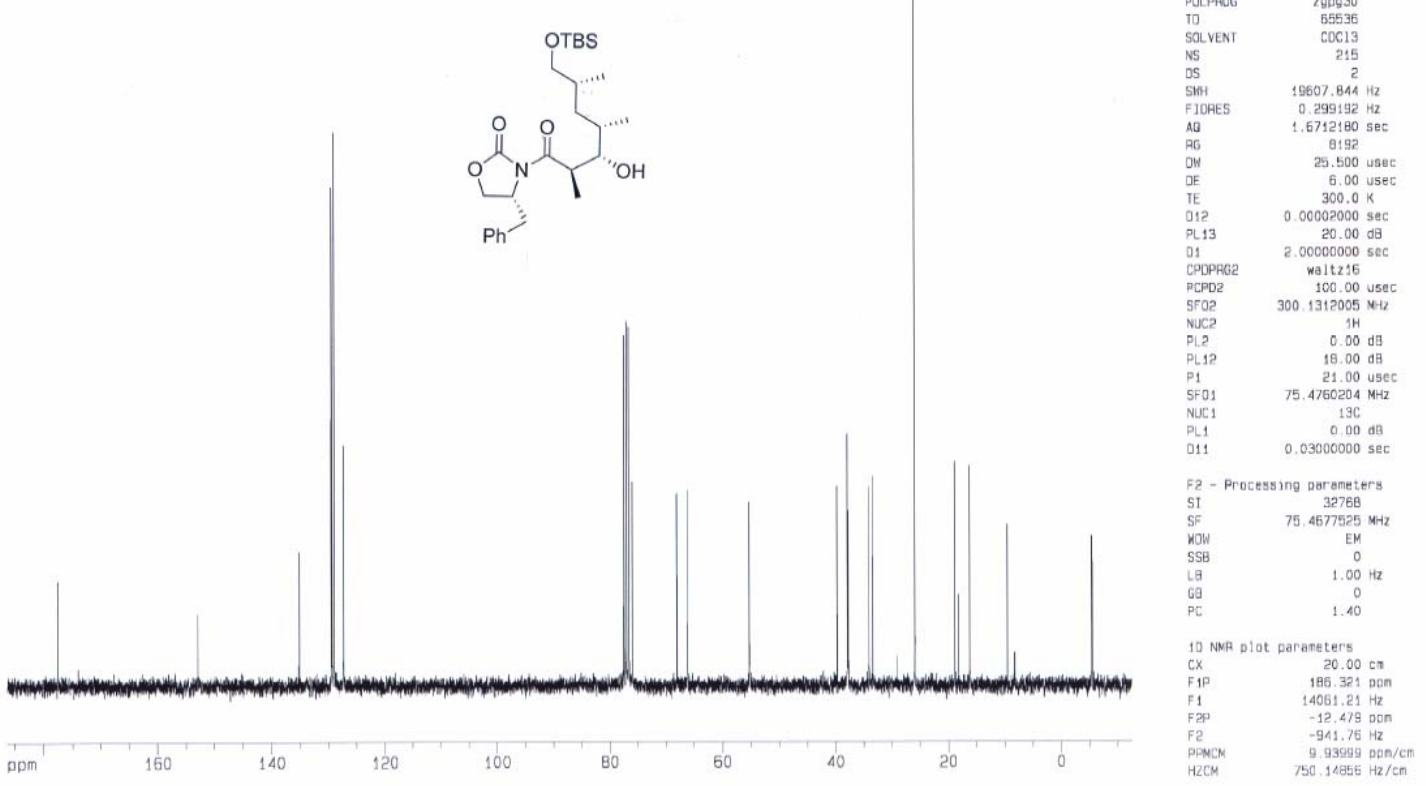




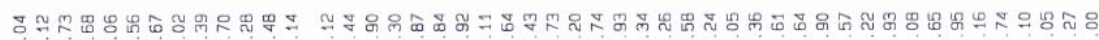

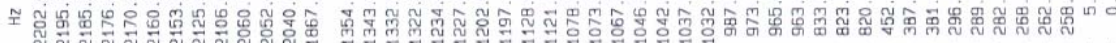

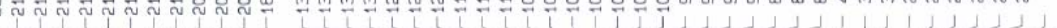

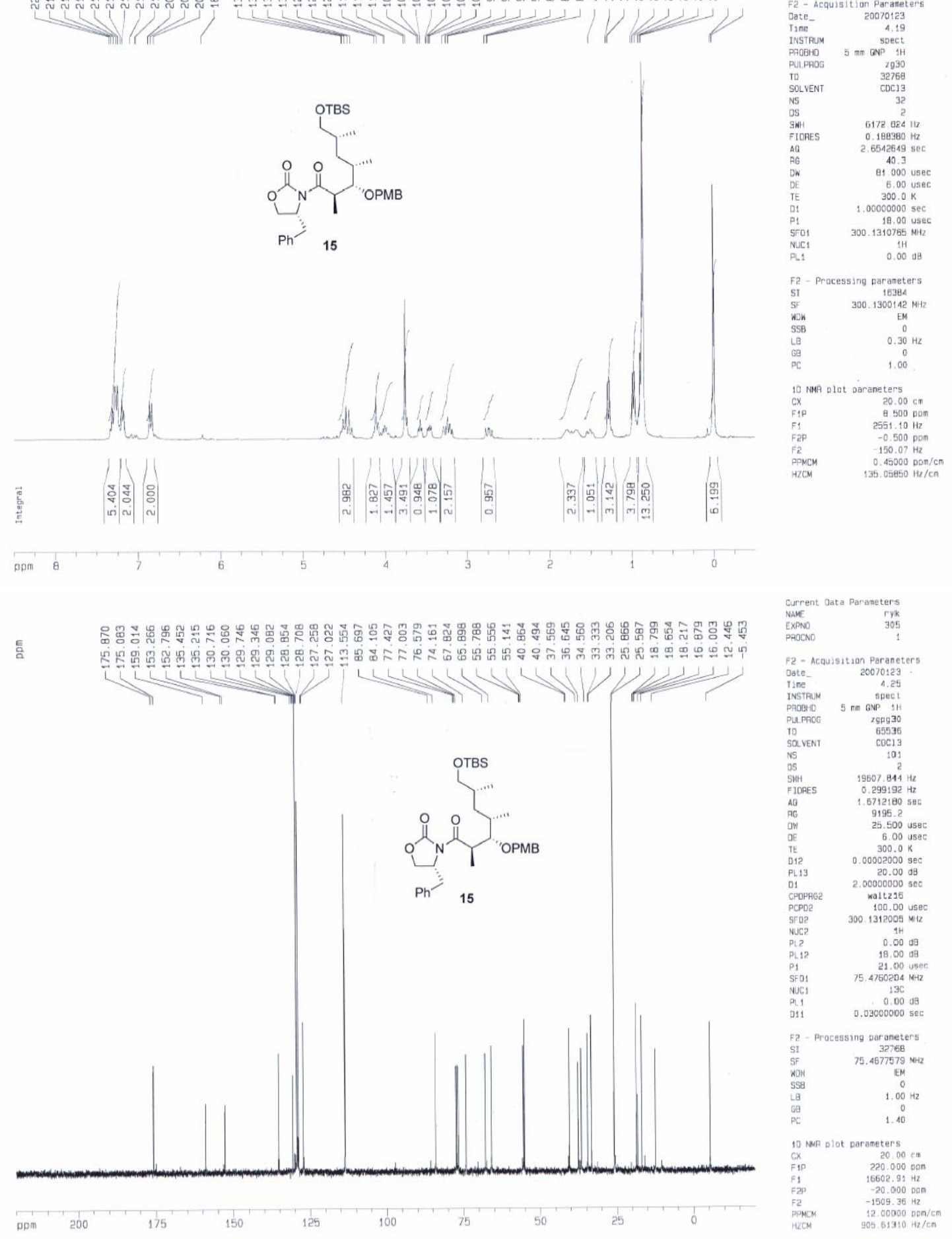



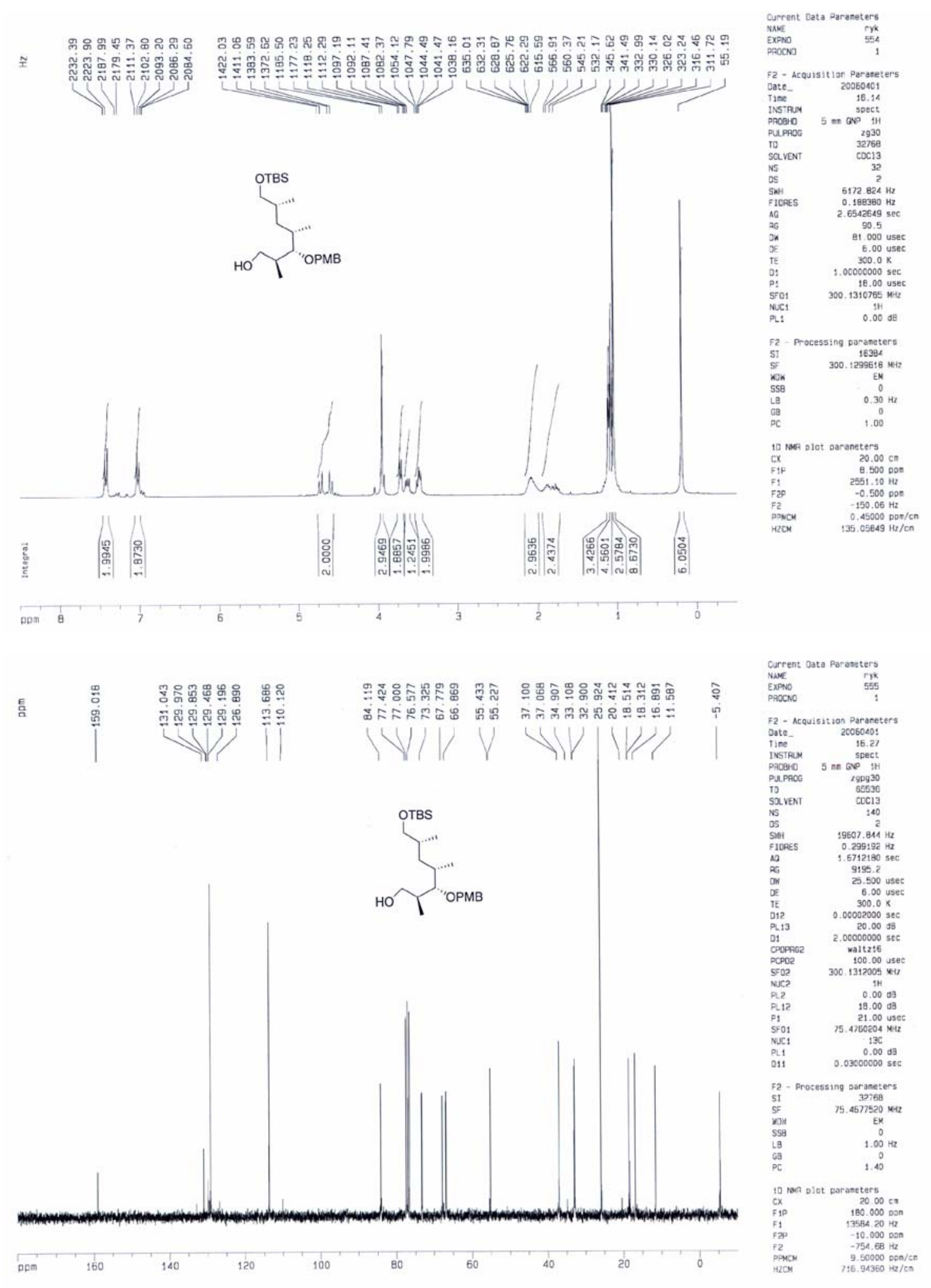


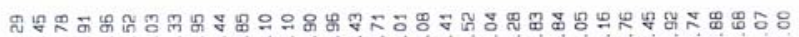

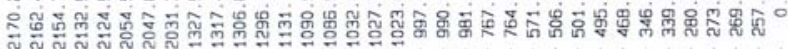
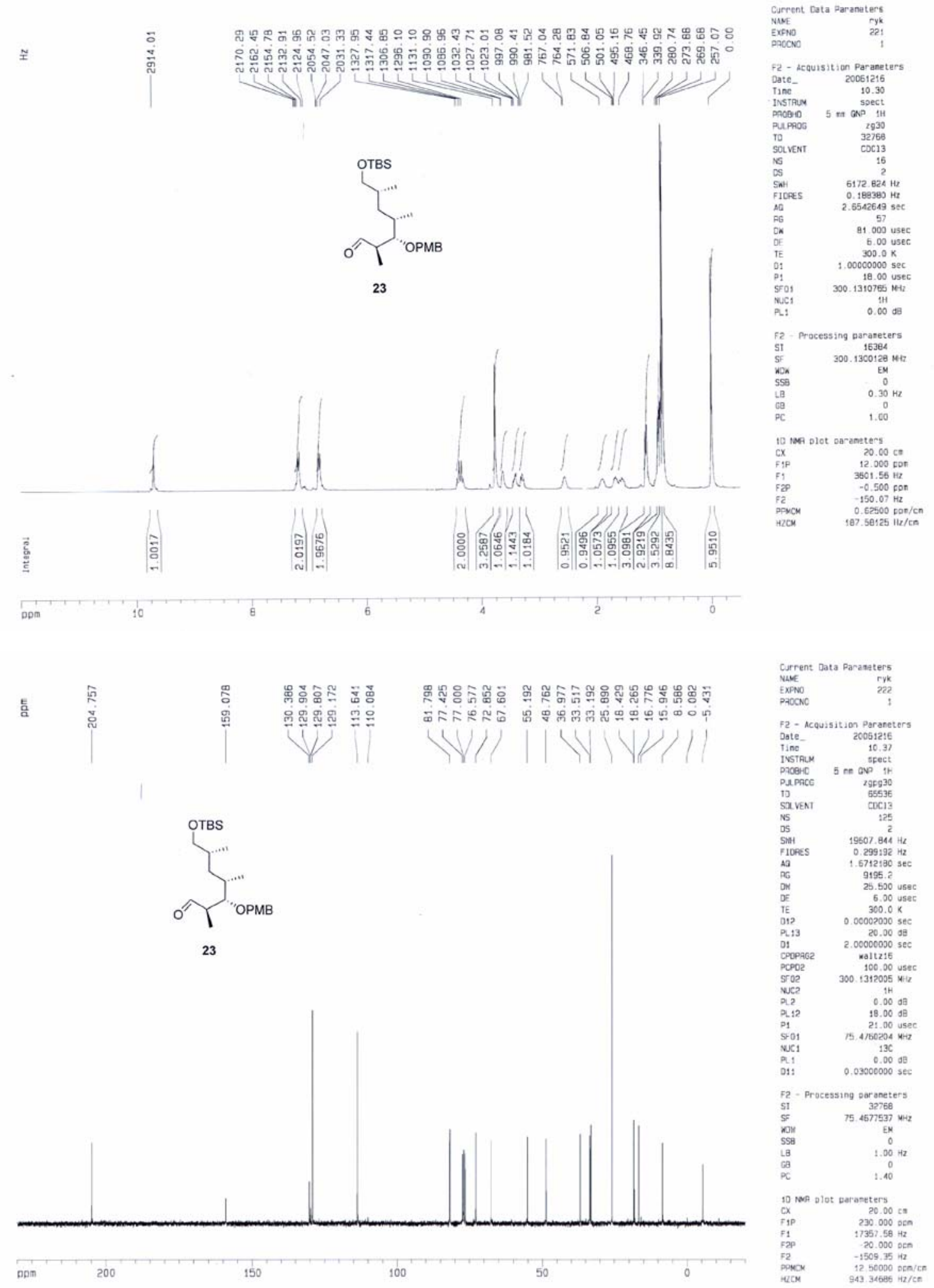


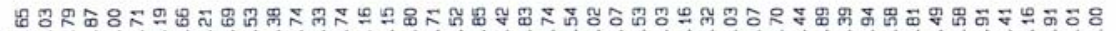

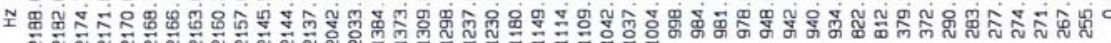
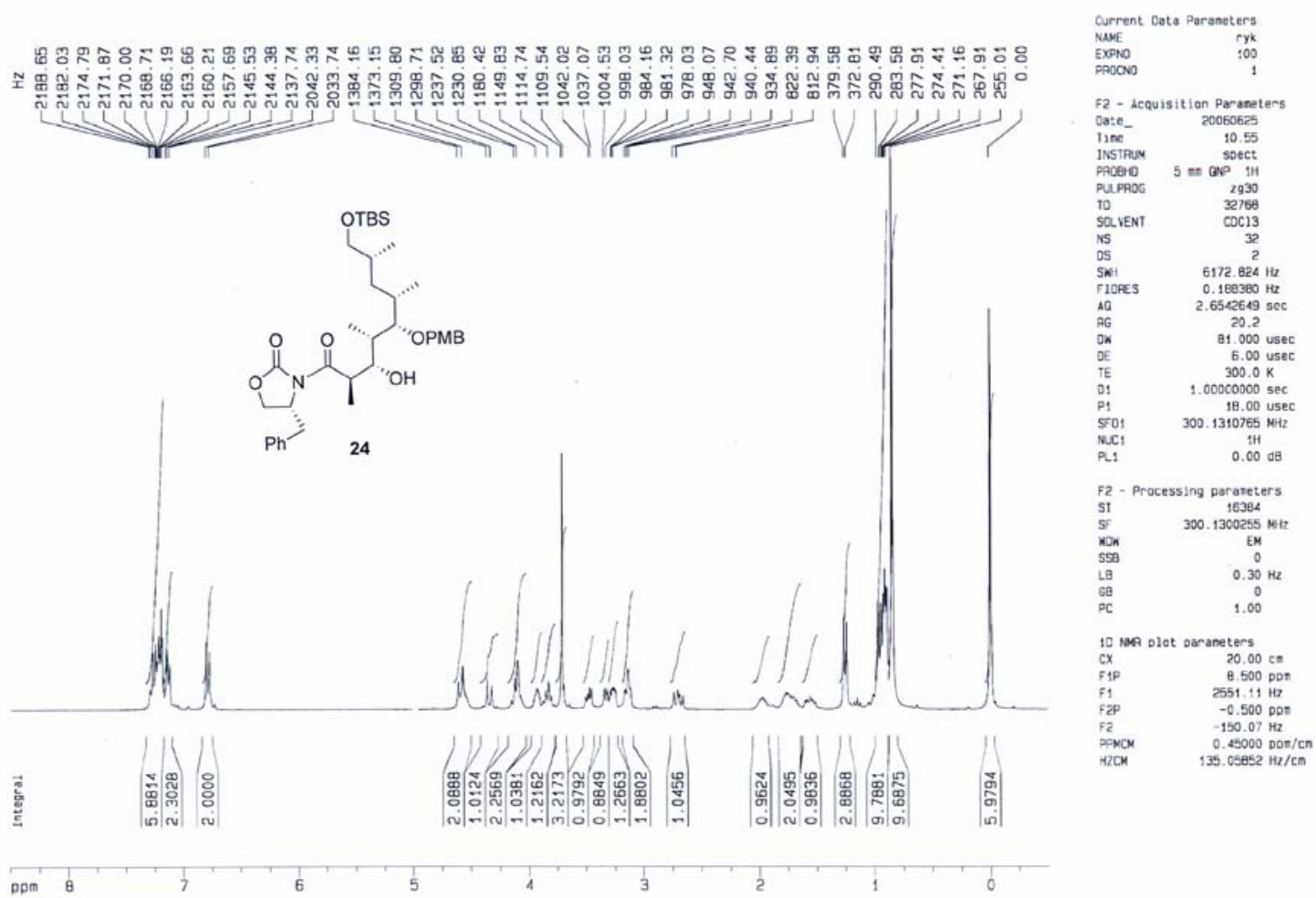

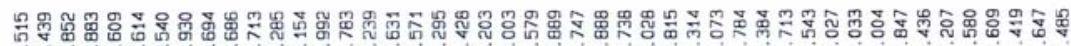

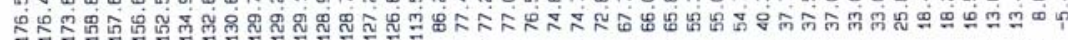

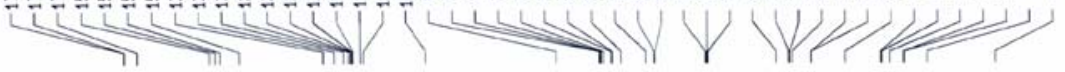<smiles>CC(CO)CC(C)C(C)C(O)C(O)C(C)C(=O)N1C(=O)OCC1c1ccccc1</smiles>

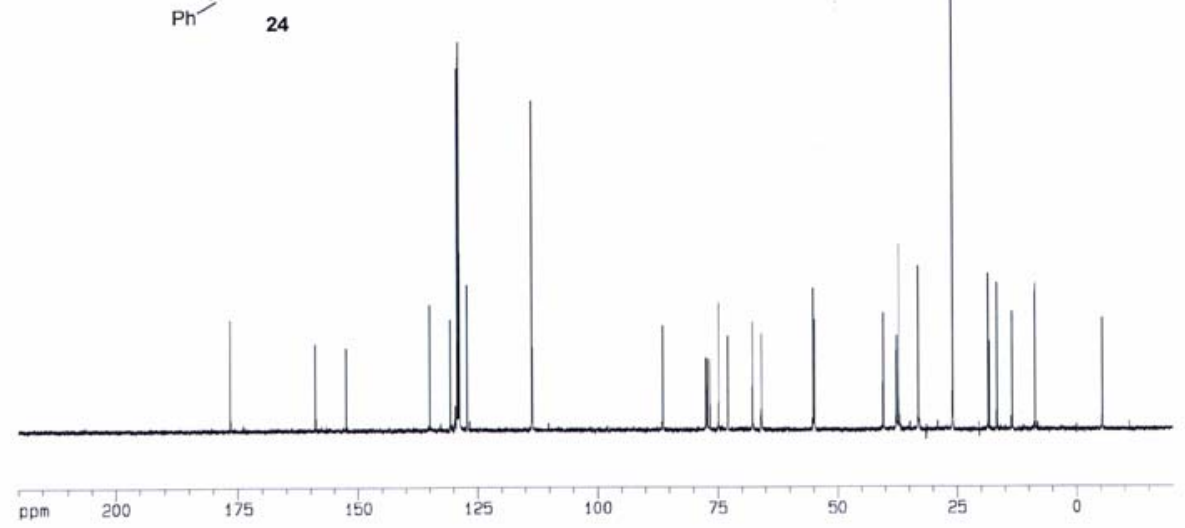




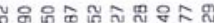

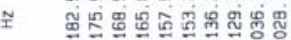

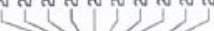

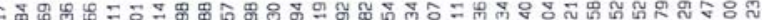

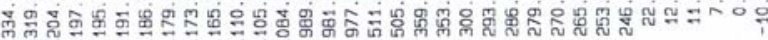
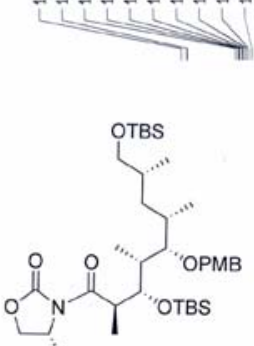

$\mathrm{Ph}^{2} 25$

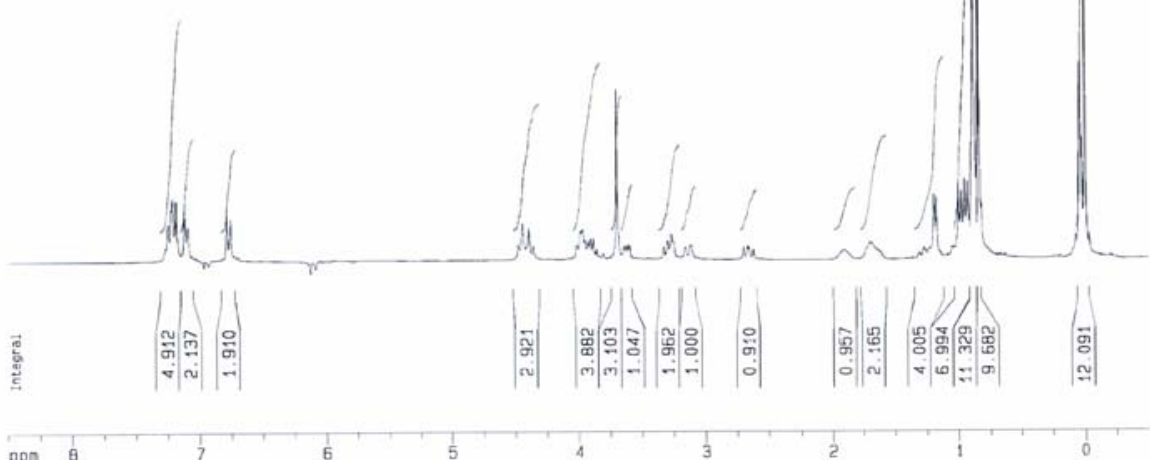

(1)

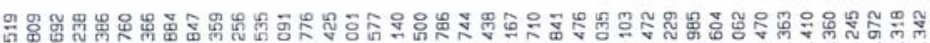
ค่
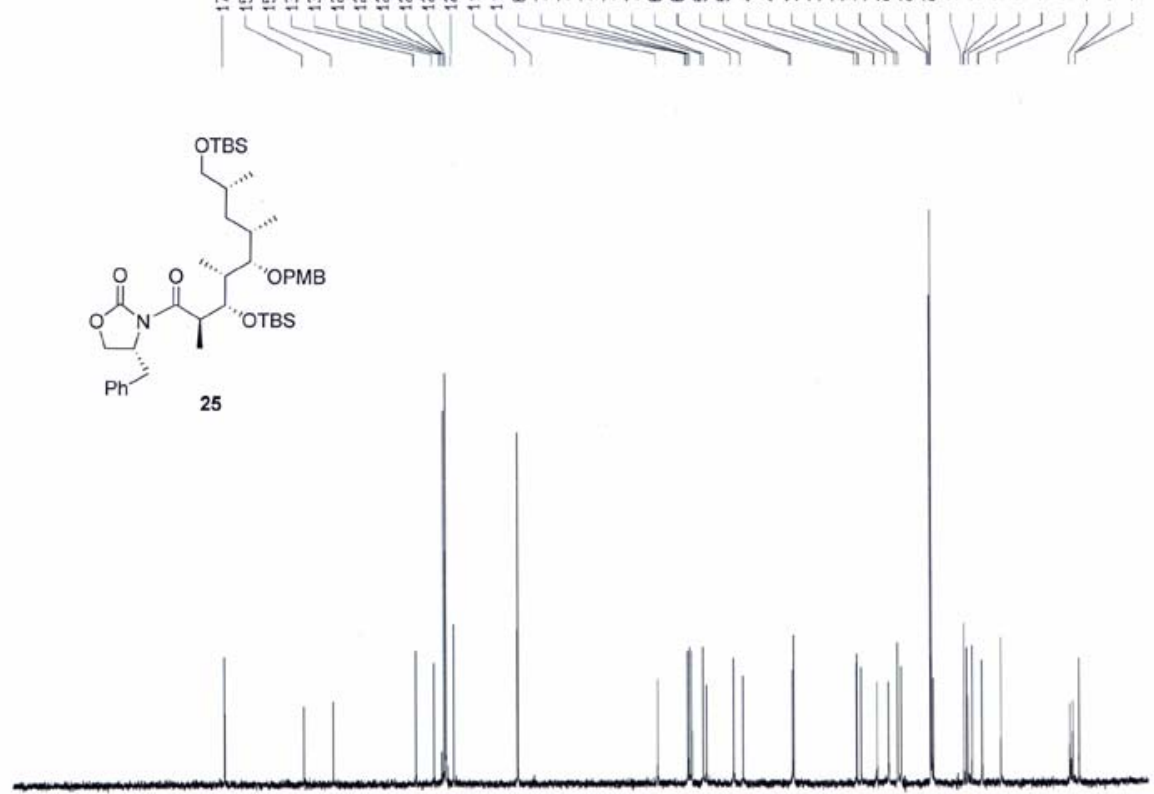

(t)

200

175

150

100

50

25

o

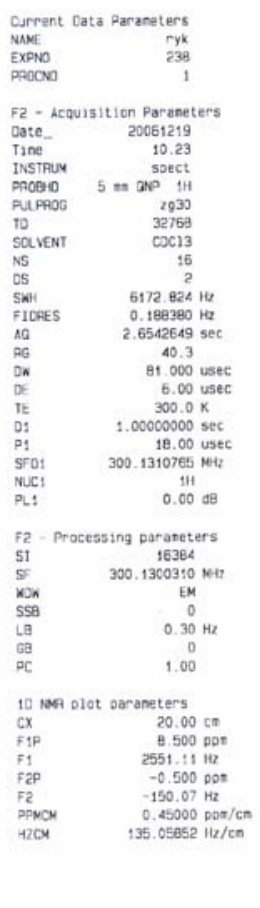

EXPNO

F2- Mequisition Paraneters
Date- 20051219

Date-
Tine
INSTR

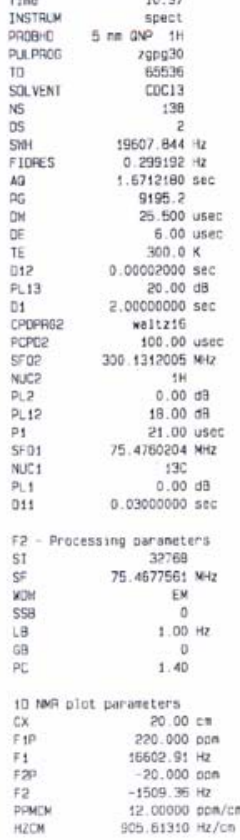




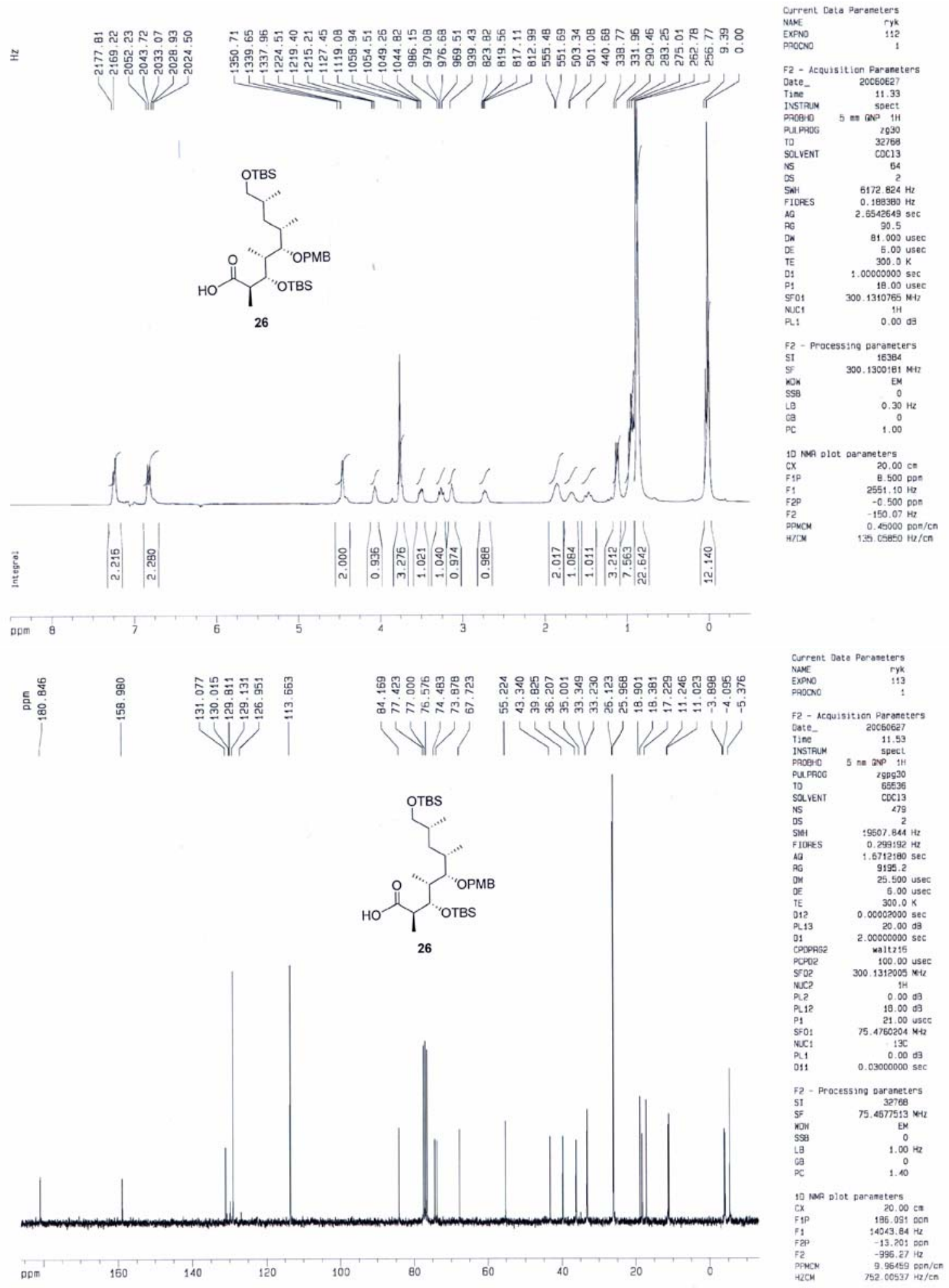




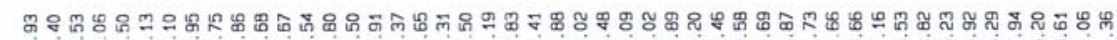

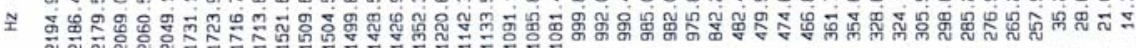

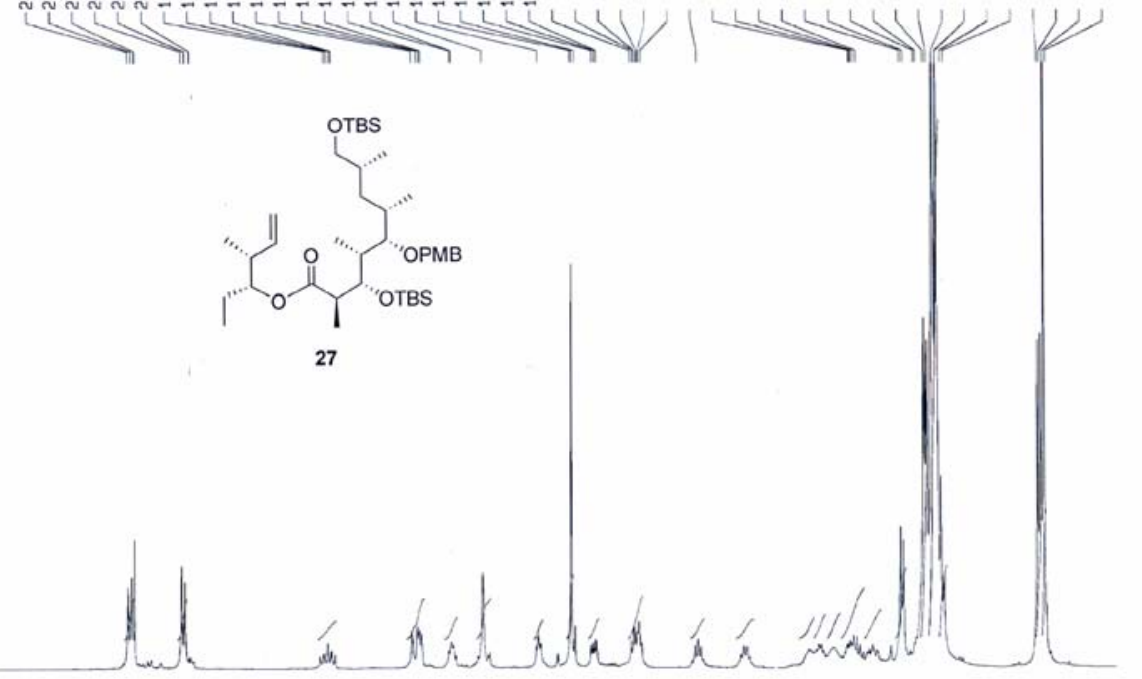

Wurrent Dats Poraneters

ExpNo

F2 - Aequis:tion Paraneters

20060630

INSTRUM SDect

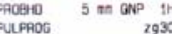

$\begin{array}{lr}\text { FULFAOG } & \text { 2930 } \\ \text { To } & 32769 \\ \text { SOLVENT } & \text { COC13 } \\ \text { NS } & 64\end{array}$

$\begin{array}{lr}\text { NS } & 64 \\ \text { OS } & 2 \\ \text { SNEt } & 6172.824 \mathrm{~Hz}\end{array}$

FIOFES
$20.186380 \mathrm{~Hz}$
$2.6542699 \mathrm{scc}$

AG
ON
DE

$300.0 \mathrm{~K}$
TE
01

\begin{tabular}{ll}
18.00 use \\
\hline 51
\end{tabular}

Ne.1 0.0000

F2 - Processing paraneters
S1 16304
SF

SSB

$6 \mathrm{C}-1.00$

10 nop olot oaraneters
CX $20.00 \mathrm{~cm}$

8.500 por

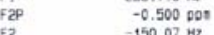

$\begin{array}{ll}\text { PPNCM } & 0.45000 \mathrm{Das} / \mathrm{cm} \\ \text { HZCM } & 135.05850 \mathrm{~Hz} / \mathrm{Cm}\end{array}$

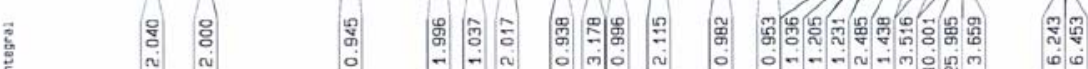

opm 8

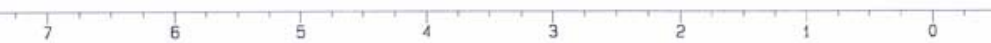

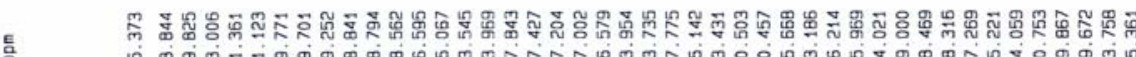

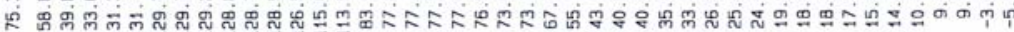

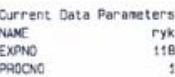
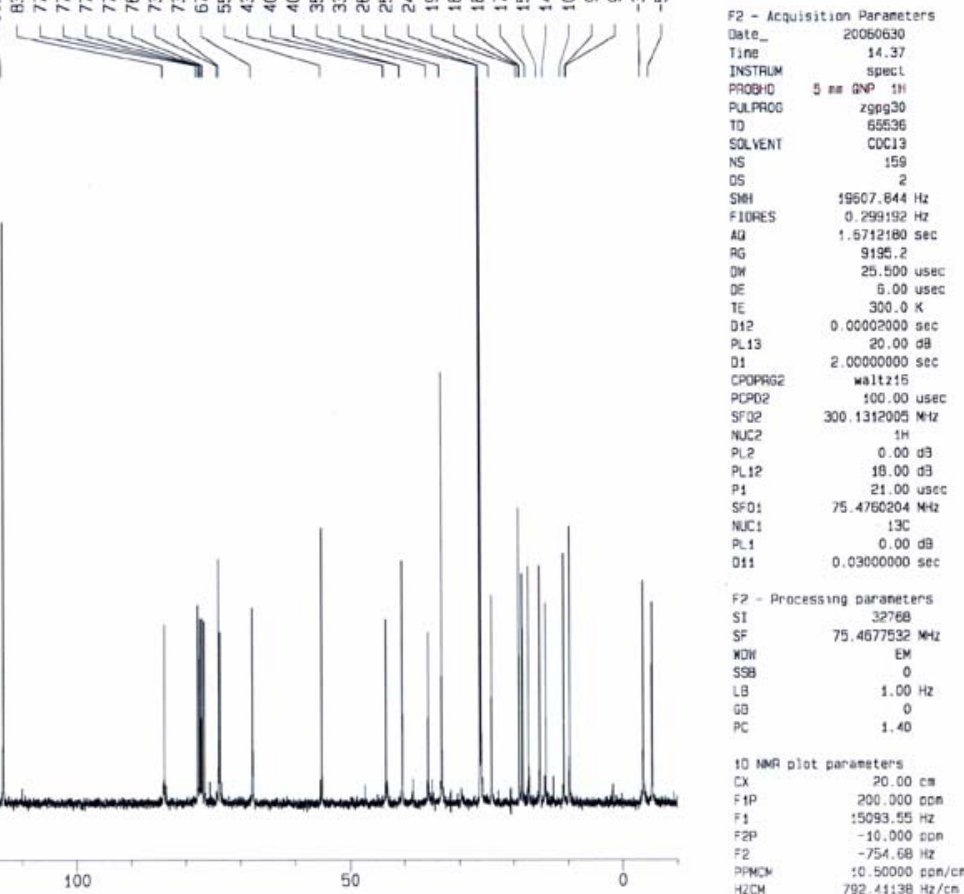


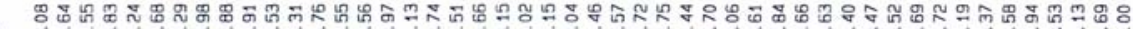

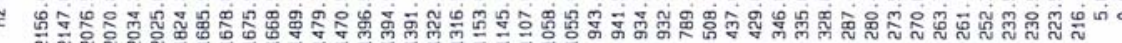
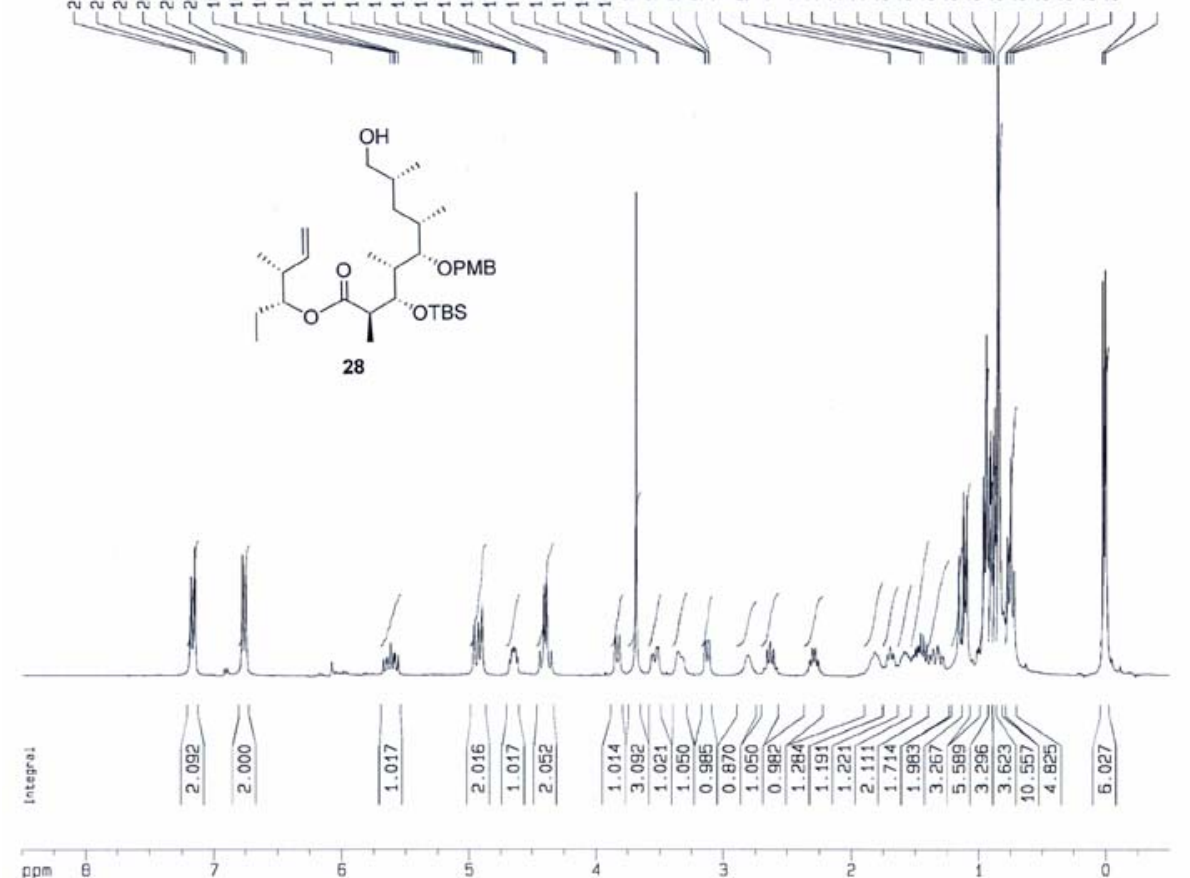

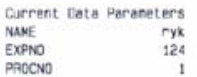

F2 - Acquisition Pareneters

Date- 20060702

INSTRUM $5 \mathrm{manO}$ Speet:

2930
PUDAROG
TD

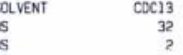

$\begin{array}{ll}\text { SAH } & 6172.824 \mathrm{~Hz} \\ \text { Filots } & 0.188380 \mathrm{~Hz}\end{array}$

AQ

$\begin{array}{cc}\text { Dn } & 81.000 \text { usec } \\ D E & 5.000 \text { usec } \\ \text { TE } & 300.0 \mathrm{~K}\end{array}$

$\begin{array}{ll}\text { TE } & 1.0300 .0 \mathrm{~K} \\ 01 & 1.00000000 \mathrm{sec} \\ 01 & 18.00 \mathrm{usec}\end{array}$

S501 $300.1310765 \mathrm{NHz}$

Ne.C: $\quad 0.00 \mathrm{~dB}$

F2 - Processing paraneters

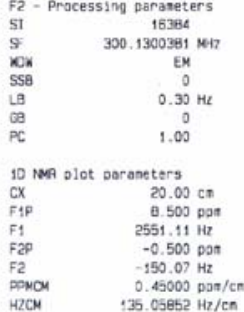

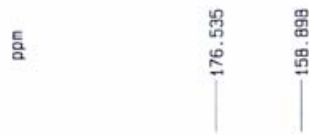

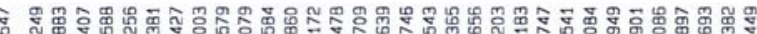

Current Date Paraneters

SPNO

F2 - Acquisition Paraneters

I. 9.49

$5 \mathrm{mav}$ art

$\quad 65536$
$\quad 0013$
121
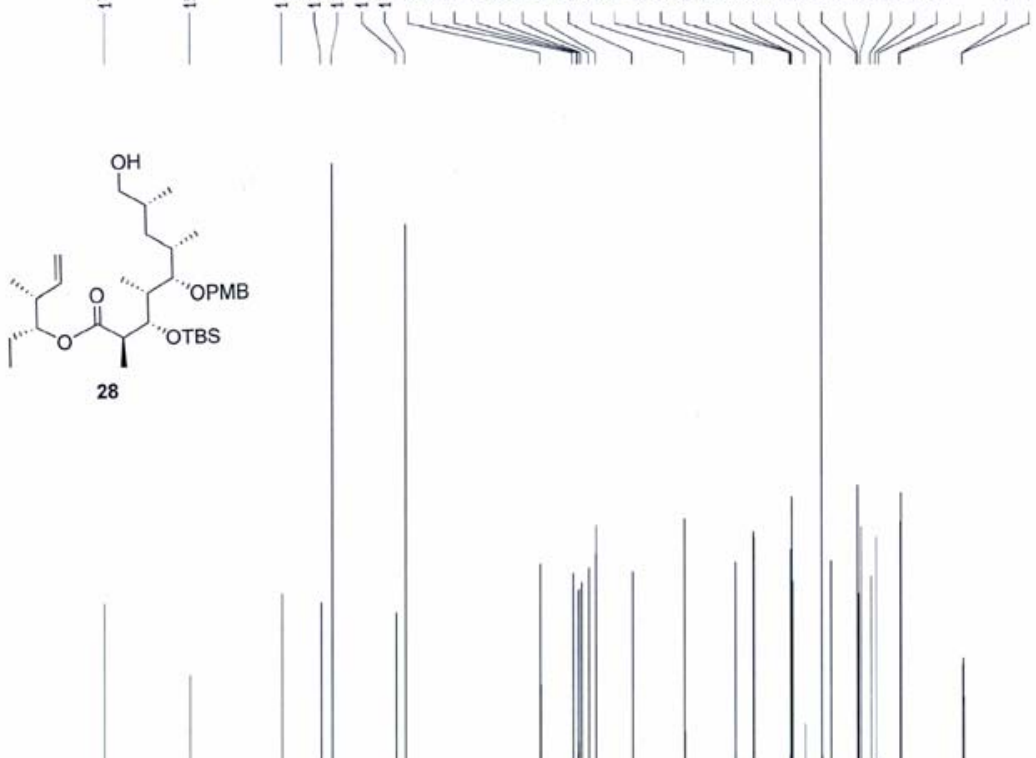

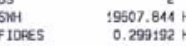

$0.299192 \mathrm{~Hz}$
$\quad 1.57129180 \mathrm{sec}$

$\begin{array}{ll}\text { R6 } & 9195.2 \\ \text { ON } & 25.500 \text { use } \\ \text { OE } & 5.00 \text { use }\end{array}$

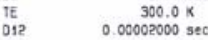

$20.00 \mathrm{~dB}$

CPOPAse $2.000000000 \mathrm{sec}$

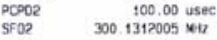

PL.? $\quad 0.00$

21.00 usec
11

PLI $\quad 0.00 \mathrm{~dB}$

2 - Prosessing paraneters

$5 \mathrm{~T} 23768$

$\begin{array}{cc}{ }_{100} & 75.4677543 \\ \text { SSB } & \text { EN } \\ 58 & 0 \\ 98 & 1.00 \mathrm{~Hz}\end{array}$

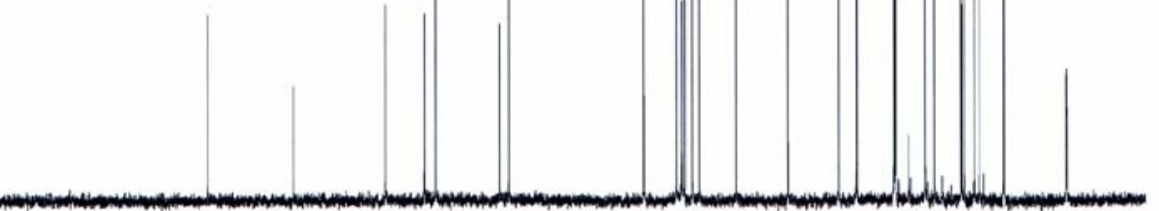

69
$P C$

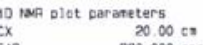

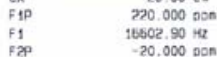

$\begin{array}{ll}\text { F2 } & -1509.75 \mathrm{~Hz} \\ \text { P.MOM } & 12.000000000\end{array}$ 


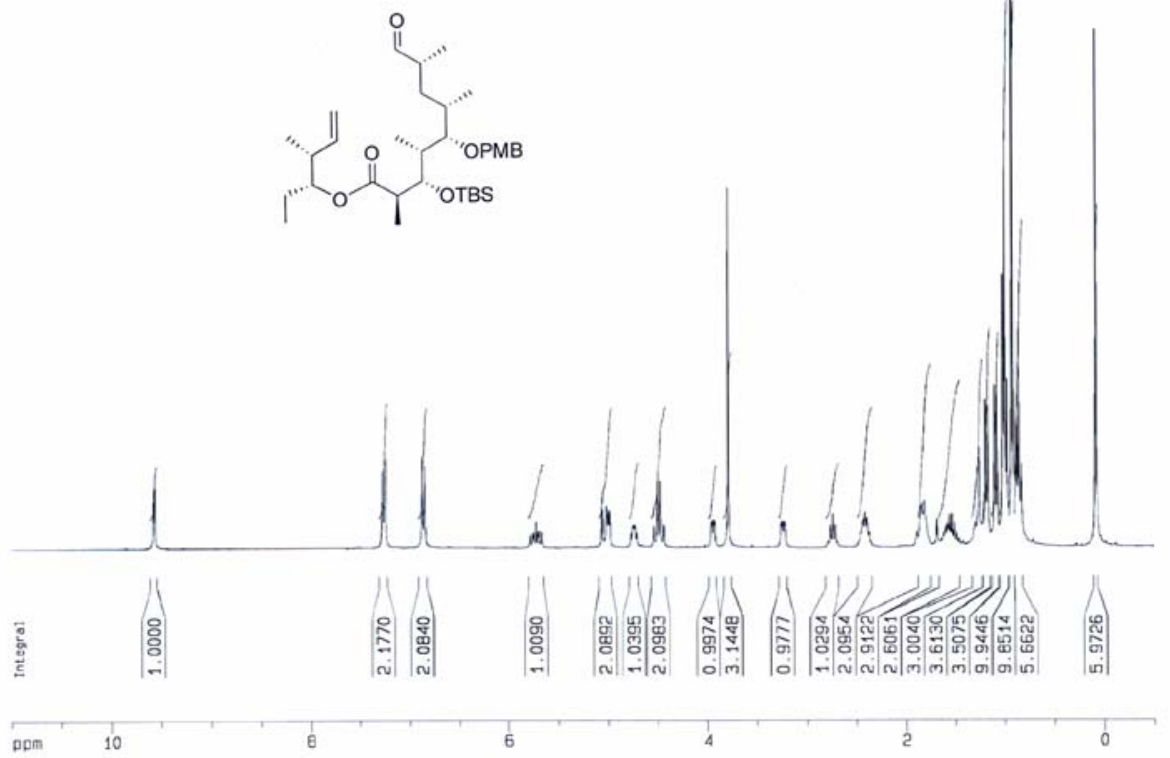

F2 - Aequisition Parameters
Date- 20060703
Tine

15.06
spect

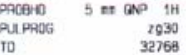

30
SOLVNT

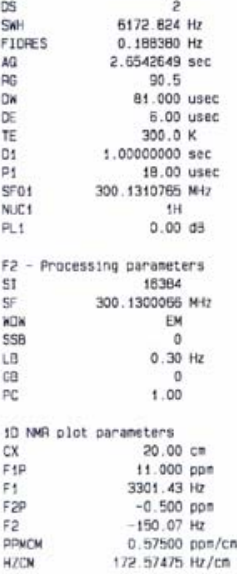

高

స

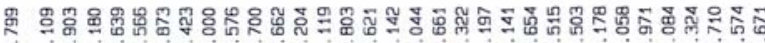

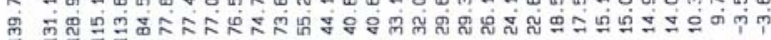

Current Data Paraveters

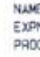

F2- Accuisistion paraneters

Date-
Tine

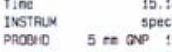

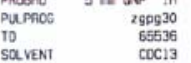

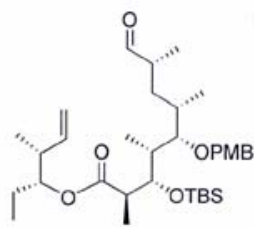

OS 19607.844

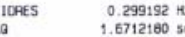

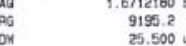

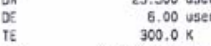

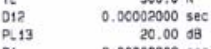

2.000000000 sec

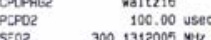

$5=02 \quad 300.1312005$ sete

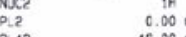

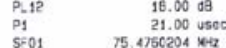

Nuc1 $\quad \begin{array}{lll}130 \\ 0.00\end{array}$

$011 \quad 0.030000000 \mathrm{sec}$

F2- Processing oaraneters
S1
S1

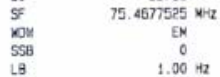

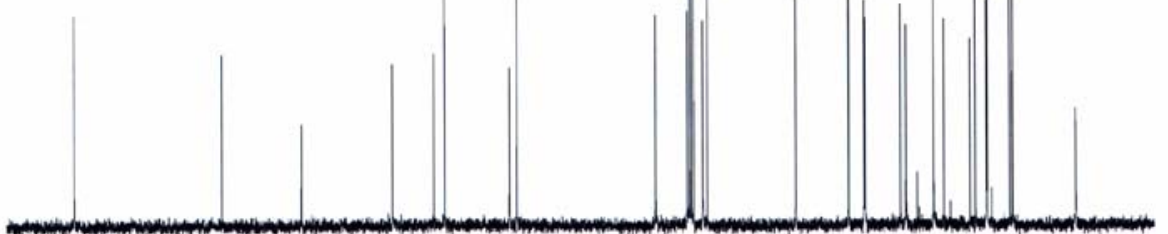

1.00
1.40

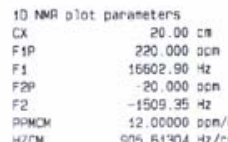




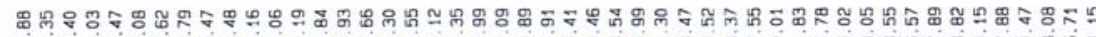

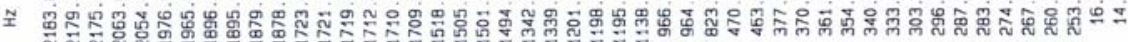
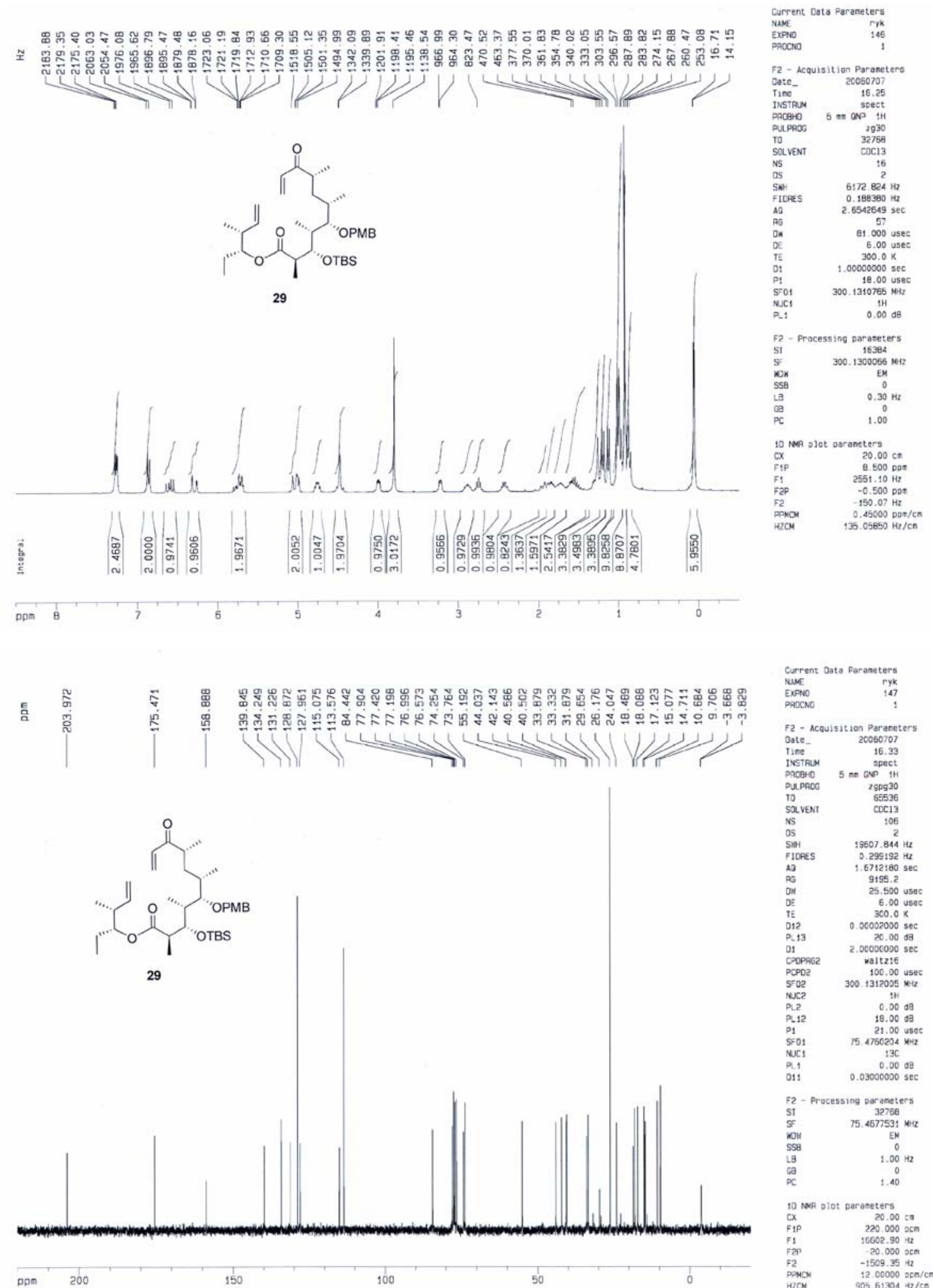

29

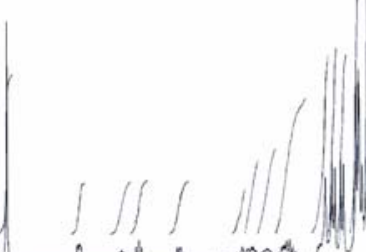

(1)

ppm 200

150

100

50

12. $00000 \mathrm{grm} / \mathrm{cm}$
$905.61304 \mathrm{~Hz} / \mathrm{cm}$ 


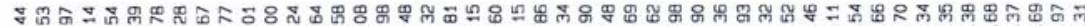

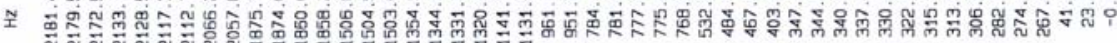

U.

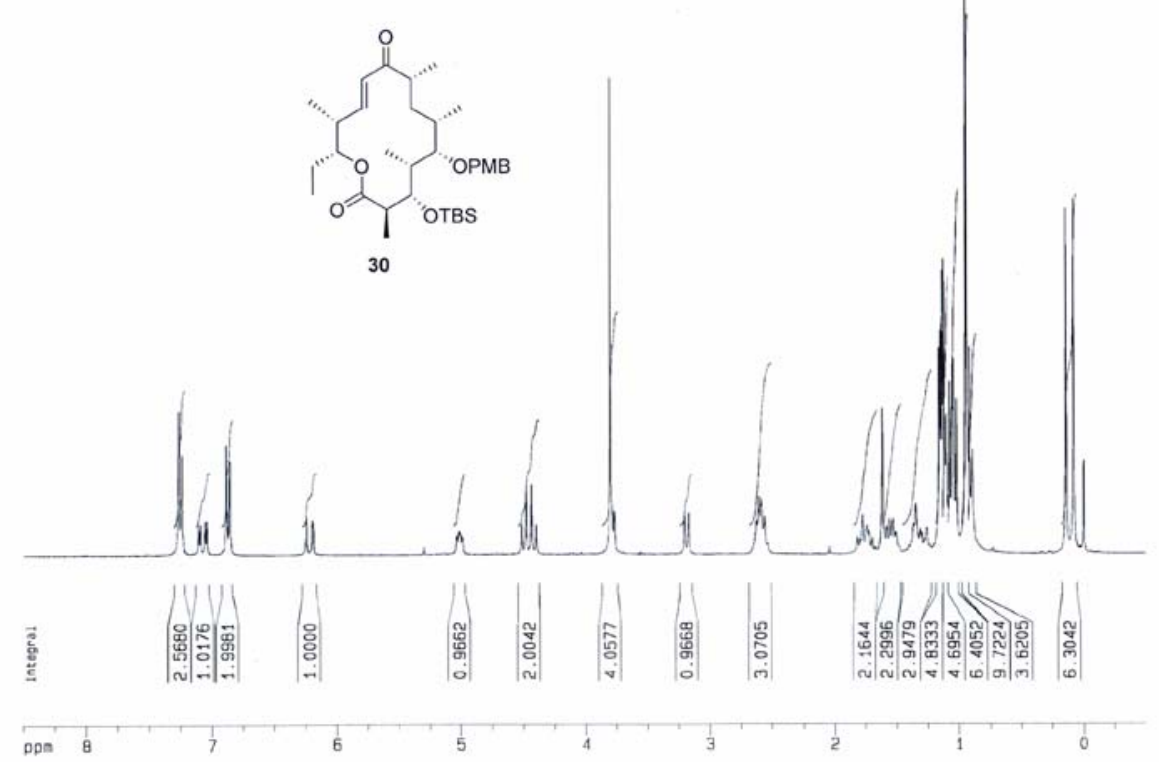

Current Data Paraneters
NuKE
EXPNO
PQOCNO

F2 - Acquisitian Paraneters

Date- 200607 as

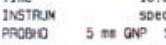

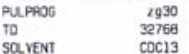

NS $\begin{aligned} 16 \\ \text { NS }\end{aligned}$

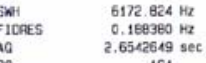

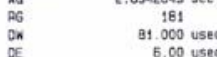

$\begin{array}{lr}\text { DE } & 5.00 \text { usec } \\ T E & 300.0 \mathrm{~K} \\ \text { TE } & 1.000000000 \mathrm{sec} \\ 01 & 18.00 \mathrm{usec}\end{array}$

$\begin{array}{lll}18.00 \mathrm{usec} \\ 5 \% 01 & 300.1310765 \mathrm{Nete}\end{array}$

N.:

F2 - Processing paraneters
SI

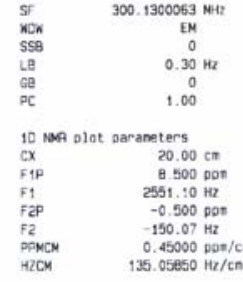

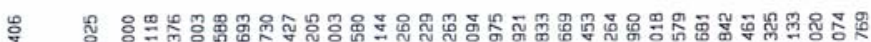

Current Dato Pareseters
ryk

EXPNO

F2 - Acquisitian Prapeters
Date- 20050726

TiNe

PROEIC 5 ne ave it

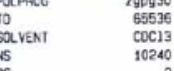

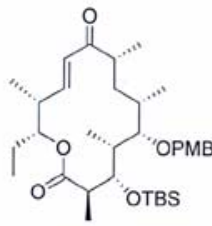

$\quad 19607.844^{2} \mathrm{He}$

$\begin{array}{ll}\text { Fiteses } & 0.299192 \mathrm{~Hz} \\ 1.6712180 \mathrm{sec}\end{array}$

$\begin{array}{ll}A 3 & 1.6712180 \mathrm{st} \\ \text { PG } & 9196.2\end{array}$

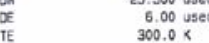

$\begin{array}{ll}T & 300.0 \% \\ 012 & 0.00002000 \mathrm{sec}\end{array}$

$\begin{array}{ll}P .13 & 20.00 \mathrm{dg} \\ 01 & 2.00000000 \mathrm{sec}\end{array}$

$\begin{array}{ll}\text { CPOPAG } & \text { xaltzit } \\ \text { PCPRe } & 100.00 \text { use }\end{array}$

$\begin{array}{ll}\text { PCPDe } & 100.00 \text { use: } \\ \text { SF02 } & 300.1312005 \text { Nite }\end{array}$

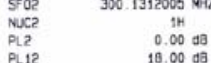

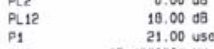

$\begin{array}{ll}\text { SF01 } & 75,4760204 \\ \text { NUC1 } & \text { 13C }\end{array}$

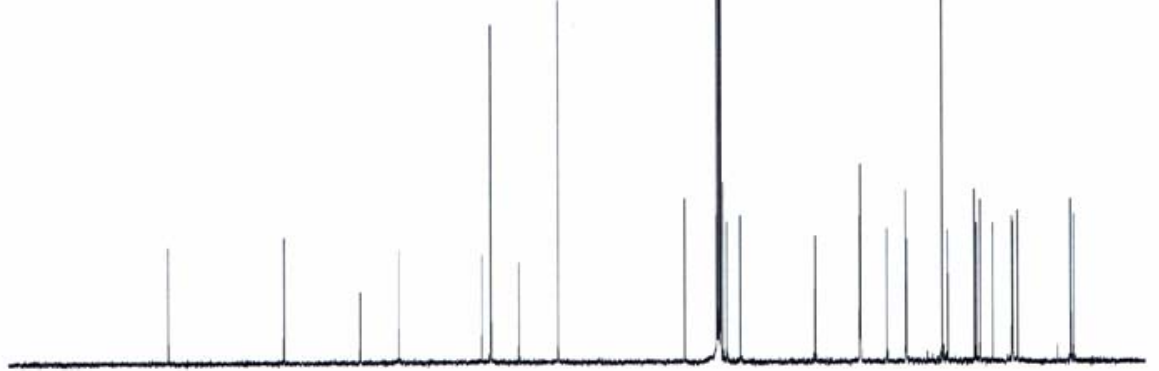

$13 \mathrm{C}$
$0.00 \mathrm{~dB}$
$0.00000 \mathrm{sec}$

2. Processing oaranezers

F2 - Processing oaranezers
SI
SF
S5

$\begin{array}{lc}\text { DON } & \text { EN } \\ S S B & 0 \\ L B & 1.00 \mathrm{~Hz} \\ G B & 0\end{array}$

10 Nof D plot paraseters
Cx $20.00 \mathrm{co}$

$\begin{array}{lr}C x & 20.00 \mathrm{co} \\ F 1 P & 240.000 \mathrm{Don} \\ F_{1} & 18112.26 \mathrm{~Hz}\end{array}$

$\begin{array}{ll}720 & -20.000 \mathrm{OPh} \\ F 2 & -1509.35 \mathrm{~Hz}\end{array}$

pon

200

150

100

50

13. $00000 \mathrm{pre} / \mathrm{CE}$
ge1. $08075 \mathrm{~Hz} / \mathrm{Cn}$ 


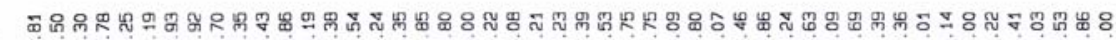

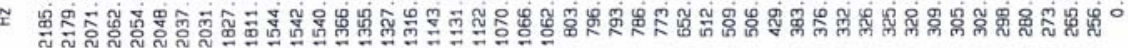

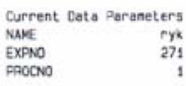

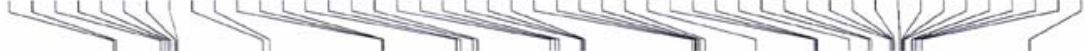

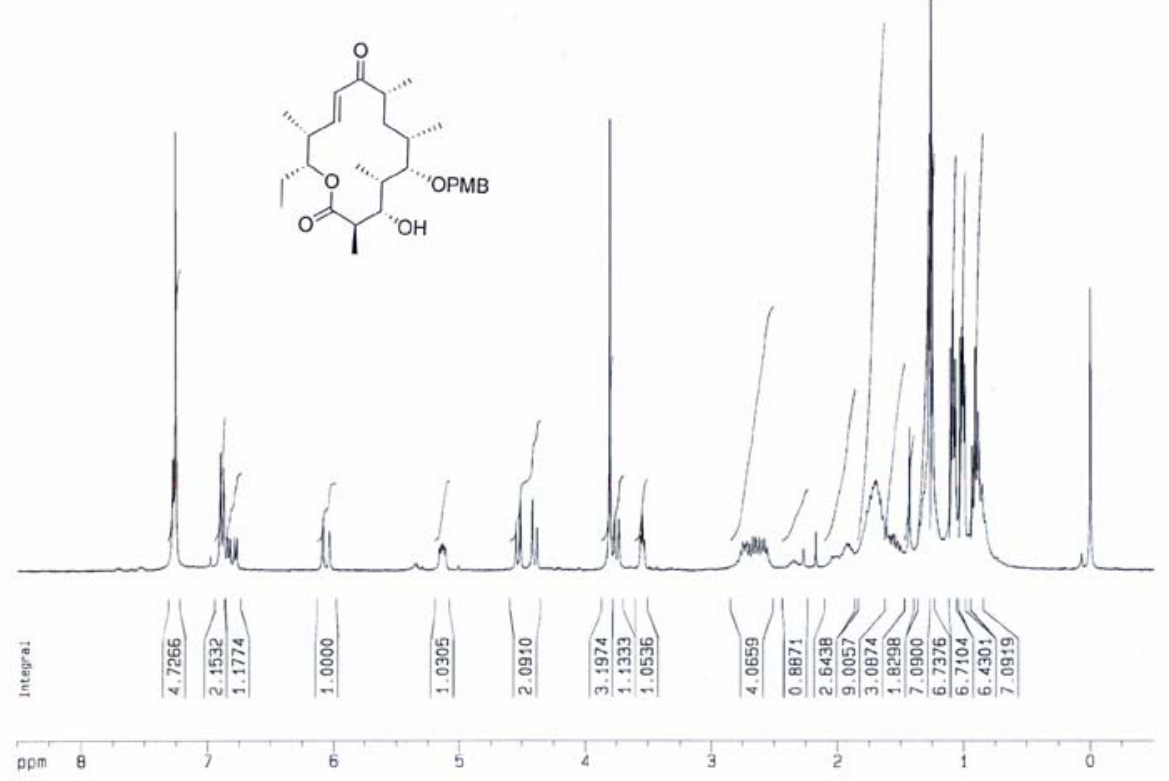

F2 - Aequisition Parameters

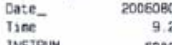

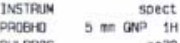

$\begin{array}{lr}\text { PULPAOG } & 2930 \\ \text { TO } & 32763 \\ \text { SOLVENT } & \text { COC13 } \\ \text { NS } & 32\end{array}$

os $\quad 6172.824 \mathrm{~Hz}$

$\quad 2.6542649 \mathrm{sec}$

$\begin{array}{lr}\text { RG } & 724,1 \\ \text { ON } & 81.000 \text { usec }\end{array}$

TE $\quad 300.0 \mathrm{~K}$

$1 \quad 1.00000000 \mathrm{sec}$

NuC: 1000

F2. - Pracessing parareters

S1 16304

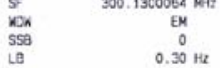

$\begin{array}{ll}\mathrm{CB} & 0 \\ \mathrm{PC} & 1.00\end{array}$

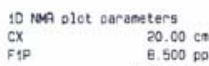

$\mathrm{Fip}$
$\mathrm{Fl}$

$\begin{array}{ll}\mathrm{Fa}^{2} & -0.500 \mathrm{mpr} \\ \mathrm{Fe} & -150.07 \mathrm{~Hz}\end{array}$

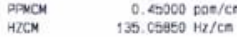

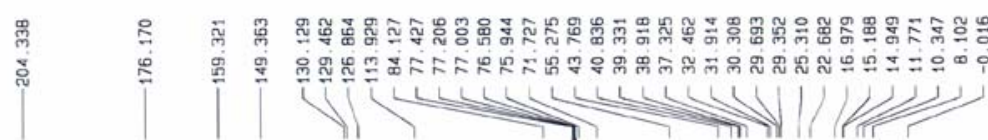

\section{Expons}

F2 - Mcquisition Parametors
Date- 20060906
Time

Tine

spect

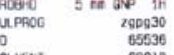

COC13
Solvent
10240
55

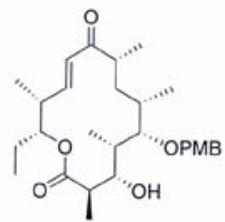

$19607.844 \mathrm{~Hz}$

\$. $6712180 \mathrm{sec}$

9196.2

25.500 use:
8.00 use:

DE
TE
TE

$0.00002000 \mathrm{sec}$
$20.00 \mathrm{dg}$
$2.00600000 \mathrm{sec}$

POFAG

100.00 use:
Fe0

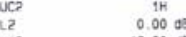

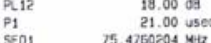

ace $\quad 0.00 \mathrm{~dB}$

$011 \quad 0.03000000 \mathrm{se}$

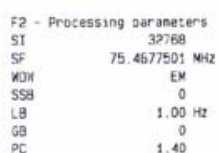

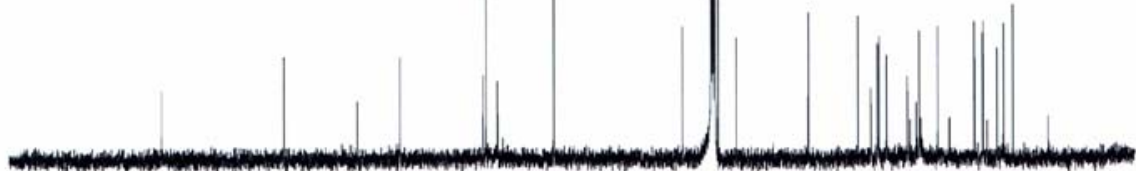

10 mar Dlot paraneters

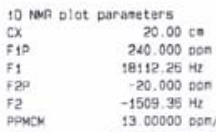

pon

200

150

100

Hitas 


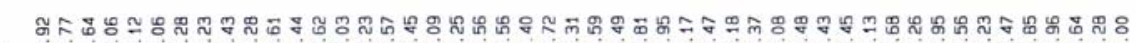
.

\begin{tabular}{l} 
Current data Paraneters \\
NaKE \\
EXPNO \\
PACONO \\
\hline 208 \\
\hline
\end{tabular}
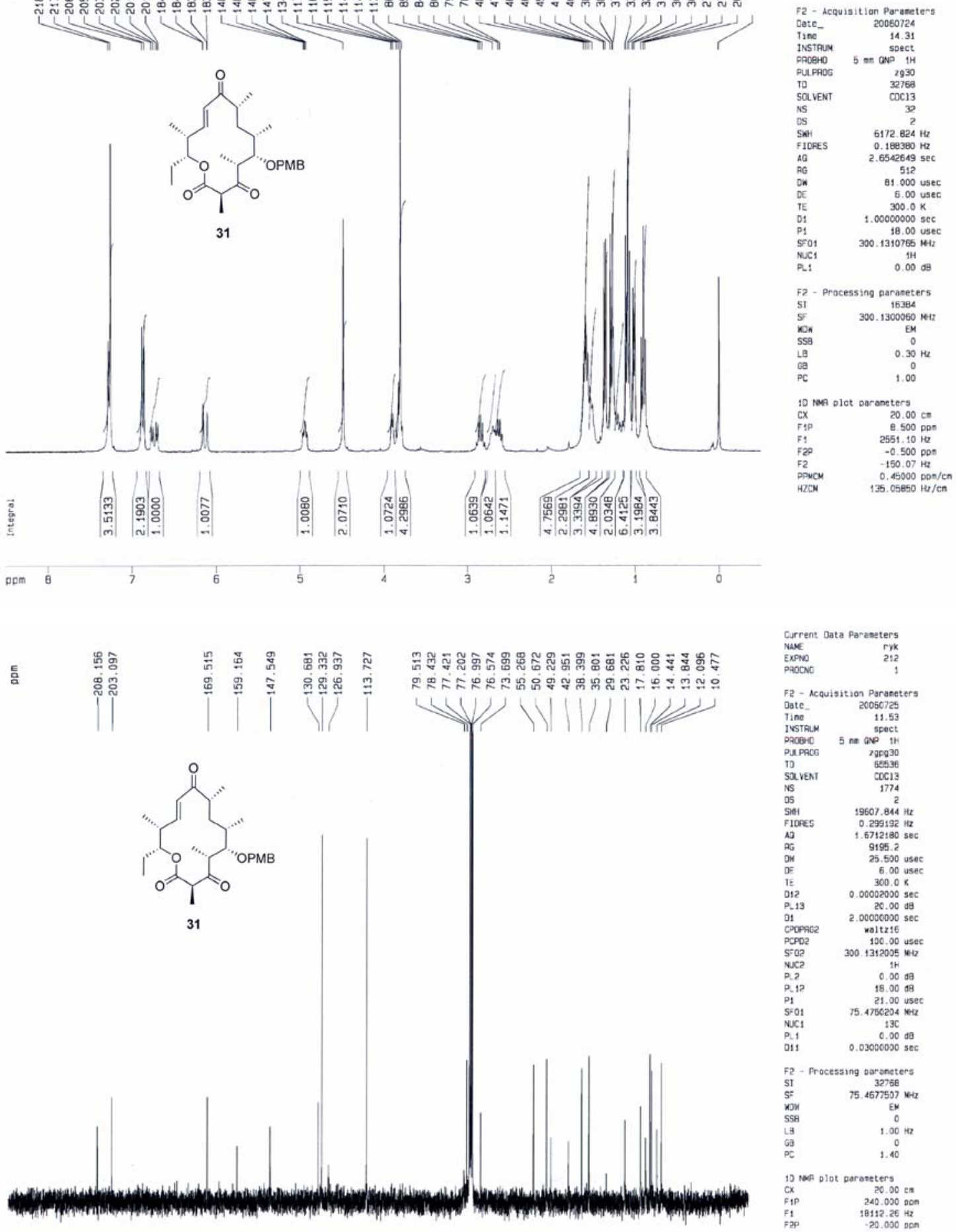

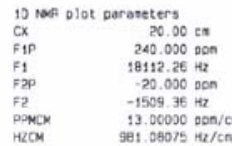



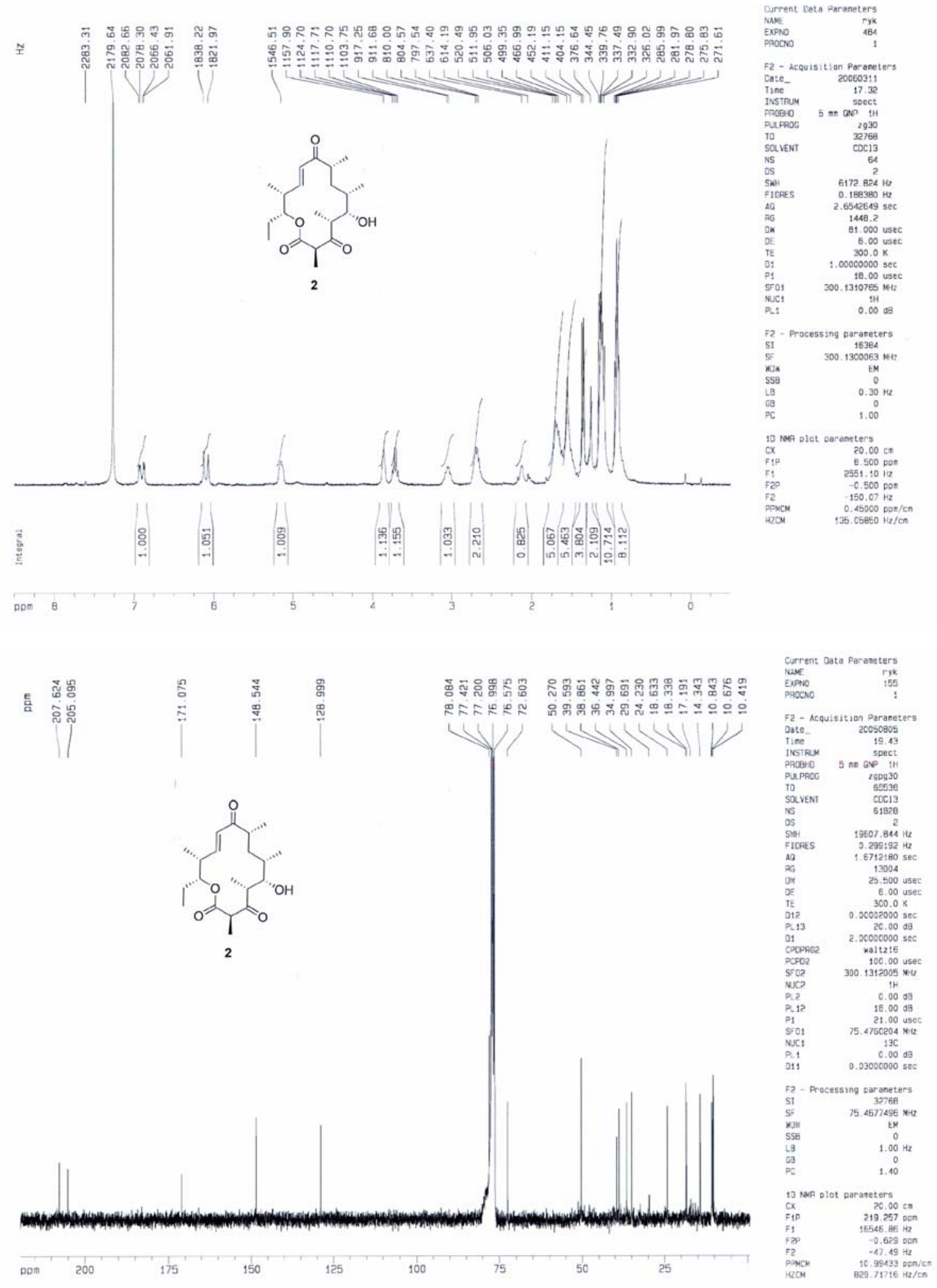